\title{
SUR LA PREMIÈRE DÉRIVÉE
}

\section{PAR \\ Z. ZAHORSKI}

1. Introduction. Le problème de caractêriser sans tautologie à l'aide des conditions topologiques et métriques la classe des fonctions réelles d'une variable réelle qui admettent une fonction primitive-n'est pas résolu. Dans ce travail je considère en général les fonctions primitives continues ayant une dérivée (finie ou infinie) en chaque point. La condition nécessaire connue qu'une telle dérivée doit être une fonction de classe I de Baire remplissant la condition de Darboux $(f(x)$ prend dans chaque intervalle $(a, b)$ toutes les valeurs intermédiaires entre $f(a)$ et $f(b)$, si $f(a) \neq f(b))$, n'est pas suffisante, comme cela résulte d'un très simple exemple de Lebesgue. Je vais citer cet exemple puisque dans la suite je donnerai des exemples pareils qui s'inspirent de celui de Lebesgue. Posons

$$
\begin{aligned}
& f(x)=\left\{\begin{array}{cll}
\sin (1 / x) & \text { pour tout } & x \neq 0, \\
0 & \text { pour } & x=0,
\end{array}\right. \\
& g(x)=\left\{\begin{array}{cll}
\sin (1 / x) & \text { pour tout } & x \neq 0 \\
1 & \text { pour } & x=0 .
\end{array}\right.
\end{aligned}
$$

Dans ce cas

$$
g(x)-f(x)=\left\{\begin{array}{lll}
0 & \text { pour tout } & x \neq 0 \\
1 & \text { pour } & x=0
\end{array}\right.
$$

La fonction $g(x)-f(x)$ ne remplit pas la condition de Darboux. Il en résulte que $f(x)$ ou $g(x)$ n'est pas la dérivée d'une fonction continue. Il est facile de donner l'exemple d'une seule fonction de ce type qui n'est pas une dérivée. J'appelle fonctions de classe $\mathcal{F}$ les fonctions de I classe de Baire remplissant la condition de Darboux. La classe $\mathcal{F}$ peut être caractérisée par les propriétés topologiques des ensembles $\{f(x)>a\},\{f(x)<a\}$. Je vais définir la suite (finie) descendante de 8 classes des fonctions $\mathcal{F}, \mathscr{X}_{0}, \mathfrak{X}_{1}, \mathscr{X}_{2}, \mathscr{X}_{3}, \mathscr{X}_{4}, \mathfrak{X}_{5}, \mathcal{A}$ mais il n'y en a que 5 différentes. Je démontrerai notamment que

$$
\mathcal{F}=\mathscr{X}_{0}=\mathscr{X}_{1} ; \mathscr{X}_{5}=\mathcal{A} ; \mathscr{X}_{n} \supset \mathscr{X}_{n+1} \quad(0 \leqq n \leqq 4) .
$$

La classe $\mathcal{A}$ soit celle des fonctions approximativement continues. Il est facile de donner des exemples que même l'appartenance à la classe la plus étroite $\mathscr{X}_{5}$ n'est pas condition suffisante pour que la fonction soit une dérivée.

Presented to the Society, April 29, 1950; received by the editors December 9, 1948 and. in revised form, June 15, 1949. 
D'autre part cette classe n'est pas assez étendue puisqu'il existe des dérivées discontinues approximativement. L'appartenance à cette classe n'est donc pas aussi la condition nécessaire. Cependant les fonctions de classe $\mathscr{X}_{5}$, qui satisfont à la condition supplémentaire d'être bornées sont, comme on le sait, des dérivées quoiqu'il soit aisé de donner aussi des exemples des dérivées non bornées approximativement continues. La classe $\mathscr{X}_{4}$ renferme toutes les dérivées bornées, elle est cependant si vaste que même la condition d'être bornée et d'appartenir à $\mathscr{X}_{4}$ ne suffisent pas pour que la fonction soit une dérivée (c'était autrement avec $\mathcal{X}_{5}$ ). D'autre part il y a évidemment des fonctions de classe $\mathfrak{X}_{4}$ (et même de $\mathfrak{X}_{5}$ ) qui ne sont pas bornées. Puisque pour les fonctions bornées l'appartenance à $\mathcal{X}_{4}$ est la condition nécessaire (mais non suffisante) et l'appartenance à $\mathcal{X}_{5}$-la condition suffisante (mais non nécessaire) pour que la fonction soit une dérivée, on aura en désignant par $B$ la classe des fonctions bornées et par $B \mathcal{D}$ la classe des dérivées bornées

$$
\mathrm{BXr}_{4} \supset \mathrm{BD} \supset \mathrm{BXX}_{5} \text {, }
$$

deux quelconques ces classes n'étant pas identiques. Je pose le problème suivant:

I. déterminer (pareillement comme $\mathcal{X}_{n}$ ) la classe $\mathcal{X}_{4,5}$ de telle façon que $B D=B x_{4,5}$.

Les dérivées finies sont fonctions de classe $\mathcal{X}_{3}$, tandis que les dérivées infinies sont de classe $\mathfrak{X}_{2}$ qui est réellement plus étroite que $\mathfrak{X}_{1}=\mathcal{F}$. Ainsi ce travail renforce le résultat élémentaire selon lequel les dérivées sont de classe $\mathcal{F}$ (I classe de Baire avec la condition de Darboux) mais ne parvient pas à caractériser les dérivées, même bornées.

Je fais reposer la classification des fonctions sur la classification des ensembles des points de l'axe des $x$ en définissant les classes des ensembles de points $M_{n}, 0 \leqq n \leqq 5, M_{n} \supset M_{n+1}, 0 \leqq n \leqq 4$. Les classes des ensembles $M_{n}$ nous servirons non seulement à la définition des classes des fonctions $\mathcal{X}_{n}$ mais aussi à l'étude des ensembles $\left\{f^{\prime}(x)>a\right\},\left\{f^{\prime}(x)=a\right\}$, etc. Il est intéressant que les propositions sur les classes de ces ensembles sont plus fortes que celles concernant les classes des fonctions $\mathscr{X}_{n}$.P.e. je ne pose pas le problème sur la classe $M_{4,5}$, la classe $M_{4}$ suffit: la condition nécessaire et suffisante pour qu'un ensemble de points soit un ensemble $\left\{f^{\prime}(x)>a\right\}$ pour une fonction continue $f(x)$ à dérivée bornée est que cet ensemble appartienne à la classe $M_{4}$. C'est la réponse à une question de M. G. Choquet $\left[5\right.$, p. 221] $\left({ }^{1}\right)$. On voit en même temps que la définition de la classe $\mathcal{X}_{4,5}$ dans le problème I ne peut pas être tout à fait analogue à celle de $\mathscr{X}_{n}$ (c-à-d. par les ensembles $\{f(x)>a\}$ seuls). J'ai publié ces résultats partiellement sans démonstration dans [34, pp. 499-500, 509510] et dans [36].

DÉsignations. $(a, b)$-intervalle ouvert $a<x<b$; je désigne aussi $(a, b)$

(1) Nombres en parenthèses s'adressent à la bibliographie à la fin du travail. 
l'intervalle $(b, a)$ si l'inégalite $a<b$ n'est pas établie. $[a, b]$-intervalle fermé $a \leqq x \leqq b ;[a]=[a, a] ;\{W(x)\}$-ensemble de tous les $x$ remplissant la condition $W(x) ; E_{x}\{W(x, y)\}$-ensemble de tous les $x$ remplissant la condition $W(x, y) ; x \in A-x$ est un élément de l'ensemble $A ; x \notin A-x$ n'est pas un élément de $A ; \phi(A)$-image de l'ensemble $A$ (c-à-d. l'ensemble de tous les $y=\phi(x)$ pour chaque $x \in A) ; A \subset B-A$ est une partie de l'ensemble $B$; $\left\{x_{n}\right\}$-une suite $x_{1}, x_{2}, \cdots ;|A|$-la mesure de Lebesgue de l'ensemble $A$; int $A$-l'intérieur de l'ensemble $A ; \bar{A}$-la fermeture de l'ensemble $A$; dist $(x, A)$-la borne inférieure de l'ensemble des nombres $|x-y|$ pour tous les $y \in A$; dist $(A, B)$-la borne inférieure des nombres $|x-y|$ pour tous les $x \in A, y \in B$; inf $A$-la borne inférieure des nombres $x \in A$; les dérivées de Dini de la fonction $\phi(x): \Phi(x)$-la dérivée supérieure, $\phi^{+}(x)$ 一dérivée de droite, $\Phi^{+}(x)$-dérivée supérieure de droite.

2. Les conditions nécessaires. L'ensemble $E$ du type $F_{\sigma}$ n'étant pas vide, je dis qu'il remplit la condition $M_{0}$ (qu'il appartient à la classe $M_{0}$ ) si tout point $x \in E$ est un point d'accumulation bilatéral de l'ensemble $E$; la condition $M_{1}$ si tout $x \in E$ est un point bilatéral de condensation de l'ensemble $E$; la condition $M_{2}$ si chaque voisinage unilatéral de tout $x \in E$ renferme une partie de l'ensemble $E$ de mesure positive; la condition $M_{3}$ s'il existe une suite des ensembles fermés $\left\{F_{n}\right\}$ et une suite de nombres $\left\{\eta_{n}\right\}, 0 \leqq \eta_{n}<1$, tels que $E=\sum_{n=1}^{\infty} F_{n}$ et que pour chaque $x \in F_{n}$ et tout $c>0$ il existe un nombre $\epsilon(x, c)>0$ jouissant de la propriété suivante: pour tous les $h$ et $h_{1}$ tels que $h h_{1}>0, h / h_{1}<c,\left|h+h_{1}\right|$ $<\epsilon(x, c)$ on $a$

$$
\frac{\left|E\left(x+h, x+h+h_{1}\right)\right|}{\left|h_{1}\right|}>\eta_{n}
$$

la condition $M_{4}$ si $E$ remplit $M_{3}$ et $\eta_{n}>0$ pour tous les $n$; la condition $M_{5}$ si tout point $x \in E$ est un point de densité de l'ensemble $E$. En outre je dirai qu'un ensemble vide remplit toutes ces conditions. Les conditions $M_{0}$ et $M_{1}$ sont purement topologiques; il n'en est pas ainsi pour les conditions suivantes.

Il est facile de remarquer que si l'ensemble $E$ remplit $M_{n+1}$, il remplit $M_{n}$; il existe cependant les ensembles remplissant $M_{n}$ mais non $M_{n+1}$ $(n=0,1,2,3,4)$. En particulier (contrairement à ce qui a lieu pour les classes des fonctions $\left.\mathcal{X}_{n}\right) M_{0} \neq M_{1}$.

Je dis que la fonction $f(x)$ appartient à la classe $\mathcal{X}_{n}$ si pour tout a l'ensemble $\{f(x)>a\}$ appartient à $M_{n}, f(x)$ appartient à la classe $\cdot \mathcal{X}_{n}$ si-f(x) appartient a $\mathscr{X}_{n}$. Enfin je définis $\mathscr{X}_{n}=\mathscr{X}_{n}^{\cdot} \mathfrak{X}_{n}$. Il résulte de cette définition que $\mathfrak{X}_{n+1}$ $\subset \mathscr{X}_{n}^{\cdot}, \mathscr{X}_{n+1} \in \mathcal{X}_{n}, \mathscr{X}_{n+1} \subset \mathcal{X}_{n}(0 \leqq n \leqq 4)$. Je laisse au lecteur de trouver des exemples faciles montrant que $\mathscr{X}_{5} \neq \mathscr{X}_{4} \neq \mathscr{X}_{3} \neq \mathscr{X}_{2} \neq \mathscr{X}_{1}$. Par contre je vais démontrer que les classes $\mathcal{H}_{1}$ et $\mathfrak{X}_{0}$ sont identiques ce qui nécessite quelques lemmes.

LEMmE 1. Si tous les ensembles $E_{k}$ remplissent $M_{n}, \sum_{k=1}^{\infty} E_{k}$ remplit $M_{n}$. 
Je laisse au lecteur la démonstration tout à fait facile.

Lемме 2. Le produit de l'ensemble ouvert et de l'ensemble $E$ de classe $M_{n}$ est de classe $M_{n}$.

Je laisse la démonstration au lecteur en faisant dans le cas de $M_{3}$ et $M_{4}$ un peu plus difficile que les autres la remarque suivante: en désignant par $G$ l'ensemble ouvert, par $I_{k}$ les segments fermés tels que $G=\sum_{k=1}^{\infty} I_{k}$ et par $\delta(x)$ la distance du point $x \in G$ au bord de l'ensemble $G$, nous posons dans la définition de la condition $M_{3}$ (év. $\left.M_{4}\right) \epsilon_{1}(x, c)=\min [\delta(x), \epsilon(x, c)]$ au lieu de $\epsilon(x, c), F_{n, k}=F_{n} \cdot I_{k}, \eta_{n k}=\eta_{n}$ (où $E=\sum_{n=1}^{\infty} F_{n}$ ) et après avoir ordonné les suites $F_{n k}$ et $\eta_{n k}$ (évidemment de la même manière) en suites avec les indices simples, nous obtenons, puisque $E G=\sum_{n=1}^{\infty} \sum_{k=1}^{\infty} F_{n k}$, la conclusion que $E G$ remplit $M_{3}$ (évent. $M_{4}$ ).

Les lemmes suivants concernent la décomposition d'un intervalle en deux ensembles. La démonstration de ce que la fonction de classe $\mathcal{X}_{0}$ jouit de la propriété de Darboux se fait par la réduction à l'absurde en supposant que $f(x) \in \mathcal{X}_{0}, f(a) \neq f(b)$, p.ex. $f(a)<f(b)$, pour un $c \in(f(a), f(b))$ que l'ensemble $(a, b)\{f(x)=c\}$ est vide; donc $(a, b)=(a, b)\{f(x)>c\}+(a, b)\{f(x)<c\}$. On peut montrer que les deux termes de la dernière somme ne sont pas vides et sont de classe $M_{0}$. Nous avons donc obtenu une division de l'intervalle ouvert en deux ensembles disjoints non vides de classe $M_{0}$ ce qui est impossible à cause des lemmes énoncés plus bas.

Les désignations usées dans les lemmes 3-7 sont les mêmes à cause de la répétition des mêmes suppositions. Je désigne notamment par $A$ et $B$ les ensembles non vides disjoints tels que $(a, b)=A+B ;$ par $A_{1}=A-\operatorname{int} A$, $B_{1}=B-$ int $B, Q=[a]+[b]+A_{1}+B_{1}=[a, b]-($ int $A+$ int $B)$. L'ensemble $Q$ est donc fermé.

Lemme 3. Si $A+B=(a, b), A \neq 0, B \neq 0, A B=0$, l'ensemble $A_{1}+B_{1}$ n'est pas vide.

Démonstration. Supposons au contraire que $A_{1}+B_{1}=0$. Dans ce cas $A=$ int $A, B=$ int $B$, c'est-à-dire les ensembles $A$ et $B$ sont ouverts. Mais $(a, b)$ n'est pas la somme de deux ensembles ouverts disjoints non vides, d'où la contradiction, c'est-à-dire que $A_{1}+B_{1} \neq 0$ c.q.f.d.

Lemme 4. Les suppositions étant les mêmes que dans le lemme 3, si en outre $A$ et $B$ sont $d u$ type $F_{\sigma}$, il existe un tel segment fermé $I \subset(a, b)$ et un tel ensemble $C=A_{1}$ (ou év. $\left.B_{1}\right)$ que $Q$ int $I \neq 0$,

$$
Q I=C I \text {. }
$$

Démonstration. Je vais faire usage des notions: de catégorie relativisée (par rapport à l'ensemble), de la non-densité par rapport à l'ensemble et du segment de l'ensemble. (Je nomme segment de l'ensemble $Q$ le produit $P Q$, 
où $P$ est l'intervalle fermé tel que $Q$ int $P \neq 0$.) Il résulte des formules $A=(a, b)-B, B=(a, b)-A$ que $A$ et $B$ sont aussi du type $G_{\delta}$, donc $A_{1}$ et $B_{1}$ sont en même temps $F_{\sigma}$ et $G_{\delta}$. D'après le lemme $3,0 \neq A_{1}+B_{1} \subset A+B$ $=(a, b) ;$ il existe donc l'intervalle fermé $I^{\prime}$ tel que $I^{\prime} \subset(a, b),\left(A_{1}+B_{1}\right)$ int $I^{\prime}$ $\neq 0$; évidemment $I^{\prime} Q=\left(A_{1}+B_{1}\right) I^{\prime}, Q$ int $I^{\prime}=\left(A_{1}+B_{1}\right)$ int $I^{\prime}$, donc $Q$ int $I^{\prime}$ $\neq 0$. Si les deux ensembles $A_{1}$ et $B_{1}$ étaient non denses sur $I^{\prime} Q$, l'ensemble $I^{\prime}\left(A_{1}+B_{1}\right)$ serait non dense sur $I^{\prime} Q$ c'est-à-dire que $I^{\prime} Q$ serait non dense sur lui même ce qui est impossible. Il s'ensuit qu'un des ensembles $A_{1}, B_{1}$ que je désigne par $C$ est dense sur un certain segment $I^{\prime \prime} Q$ de l'ensemble $I^{\prime} Q$ $\left(I^{\prime \prime} \subset I^{\prime}\right)$; étant $G_{\delta}, C$ est de deuxième catégorie sur $I^{\prime \prime} Q$. Puisque $A_{1} \subset Q$, $B_{1} \subset Q$, nous avons

$$
C \subset Q, \quad C I^{\prime \prime} Q=I^{\prime \prime} C \subset I^{\prime \prime} Q .
$$

Mais l'ensemble $I^{\prime \prime} C$ est non seulement $G_{\delta}$ mais aussi $F_{\sigma}$ et, étant de II catégorie par rapport à $I^{\prime \prime} Q$ renferme un certain segment $I^{\prime \prime \prime} Q$ de l'ensemble $I^{\prime \prime} Q\left(I^{\prime \prime \prime} \subset I^{\prime \prime}\right)$. Il s'ensuit que $Q$ int $I^{\prime \prime \prime} \neq 0$; en posant $I^{\prime \prime \prime}=I$ nous avons $I Q \subset I^{\prime \prime} C, I Q \subset I^{\prime \prime} C I=I C$; mais il résulte de (1) que $I C \subset I Q$, donc $I Q=I C$, c.q.f.d.

CorollaIRe. En désignant par $D$ celui des ensembles $A_{1}, B_{1}$ qui est différent de $C$ nous avons

$$
\begin{gathered}
I Q=I C+I D=I C, \quad I C \cdot I D=0, \text { donc } \\
I D=0 .
\end{gathered}
$$

Pour simplifier les notations nous adopterons dans la suite que $C=A_{1}$, $D=B_{1}$ ce qui ne restreint pas la généralité puisque dans le cas contraire il suffirait de changer les notations.

Lemme 5. En adoptant les suppositions du Lemme 4 on a

$$
\text { int } I \text {.int } B \neq 0 \text {. }
$$

Démonstration. Puisque int $I \subset(a, b)=A+B$, on aura

int $I=(A+B)$ int $I=A_{1}$ int $I+$ int $A \cdot$ int $I+B_{1}$ int $I+$ int $B \cdot$ int $I$, mais d'après (2) $\left(D=B_{1}\right)$ on a

$$
B_{1} \text { int } I \subset B_{1} I=0,
$$

si donc on avait int $B$.int $I=0$, on aurait int $I=A_{1}$ int $I+$ int $A$-int $I$ $=\left(A_{1}+\right.$ int $\left.A\right)$ int $I=A$ int $I$ c'est-à-dire que $A$ Dint $I$, int $A$ Dint $I$ d'où $A_{1}=A-$ int $A \subset A-$ int $I, A_{1}$ int $I \subset$ int $I .(A-$ int $I)=0$ ce qui donne, à cause de (3), $\left(A_{1}+B_{1}\right)$ int $I=Q$ int $I=0$, ce qui est en contradiction avec le Lemme 4. Il s'ensuit que int $I$. int $B \neq 0$, c.q.f.d.

Lemme 6. Les suppositions étant celles du lemme 4, il existe l'extrémité p de 
la composante de l'ensemble int $I$.int $B$ appartenant a $A$.

Démonstration. L'ensemble ouvert non vide int $I$. int $B$ (Lemme 5) est borné. Il en résulte que les extrémités de ses composantes existent. Si ces extrémités coincidaient avec les extrémités du segment $I$ il y aurait int $I$ $C$ int $B \subset B$, donc $A$ int $I \subset A B=0$, d'où $A_{1}$ int $I=0$ à cause de $A_{1} \subset A$. Puisque (3) on aurait donc $\left(A_{1}+B_{1}\right)$ int $I=Q$ int $I=0$ ce qui est en contradiction avec le Lemme 4 . Il existe donc une telle extrémité $p$ de la composante de l'ensemble int $I$. int $B$, que

$$
p \in \text { int } I \text {. }
$$

Mais $p \notin$ int $I$. int $B$, donc $p \notin$ int $B$. Si l'on avait $p \in B$, on aurait $p \in B_{1}$ $=B$-int $B$. Il résulterait de là et de (4) que $p \in B_{1}$ int $I$ ce qui est en contradiction avec (3). Il s'ensuit que $p \in B$. Mais int $I \subset(a, b)=A+B$. Donc d'après (4) $p \in A+B$ et puisque $p \in B$, on a $p \in A$, c.q.f.d.

Lemme 7( $\left.{ }^{2}\right)$. A ucun intervalle ouvert $(a, b)$ n'est la somme de deux ensembles disjoints non vides remplissant la condition $M_{0}$.

Démonstration. Les ensembles pareils remplissent les conditions du lemme 4; en les désignant respectivement par $A, B$ et en admettant que $(a, b)=A+B$, il existe en vertu du Lemme 6 un point $p \in A$ qui est l'extrémité du segment appartenant à $B$. Il s'ensuit que $p$ n'est pas un point d'accumulation bilatéral de l'ensemble $A$ contrairement a la supposition faite que $A \in M_{0}$. Il résulte de cette contradiction que $(a, b) \neq A+B$, c.q.f.d.

THÉoRغ̀me 1. Si les ensembles $\left\{f(x)>a_{n}\right\},\left\{f(x)<b_{n}\right\}$ remplissent la condition $M_{0}$ pour les suites $\left\{a_{n}\right\},\left\{b_{n}\right\}$ dénombrables denses sur une droite (l'axe des $y$ ), $f(x) \in \mathcal{F}$. Si $f(x) \in \mathcal{F}, f(x) \in \mathcal{X}_{1}$.

Démonstration. Étant donné un nombre arbitraire $a$ il existe une suite $a_{n_{k}}>a$ telle que $a=\lim _{k \rightarrow \infty} a_{n_{k}}$. Dans ce cas $\{f(x)>a\}=\sum_{k=1}^{\infty}\left\{f(x)>a_{n_{k}}\right\}$, donc en vertu du Lemme 1, l'ensemble $\{f(x)>a\}$ pour chaque $a$ remplit $M_{0}$. Il en sera de même pour l'ensemble $\{f(x)<b\}$ pour tout $b$, d'où $f(x) \in \mathcal{X}_{0}$. Supposons que $f(x) \notin \mathcal{F}$. Les ensembles $\{f(x)>a\},\{f(x)<b\}$ sont du type $F_{\sigma}$ (cela résulte de la condition $M_{0}$ ) donc $f(x)$ est une fonction de I classe de Baire et par conséquent, car $f(x) \notin \mathcal{F}$, ne remplit pas la condition de Darboux. Il existe donc un tel intervalle $(a, b)$ que $f(a) \neq f(b)$, p.ex. $f(a)<f(b)$, et un tel nombre $c, f(a)<c<f(b)$, que $f(x) \neq c$ pour tout $x \in(a, b)$, il s'ensuit de là que $(a, b)=(a, b)\{f(x)>c\}+(a, b)\{f(x)<c\}$, où les deux termes remplissent $M_{0}$. (D'après le Lemme 2 et de ce que $f(x) \in \mathscr{X}_{0}$ ). Ils sont évidemment disjoints. Ils sont non vides. En effet, puisque $b \in\{f(x)>c\}, a \in\{f(x)<c\}$ et

(2) Le problème de la décomposition (Lemme 7) fût posé par moi à Lwów au mois de mars 1941 à la réunion des mathéma ticiens de Lwów. Le lendemain il fût résolu indépendamment par S. Banach et M. Wojdysławski. La démonstration donnée ici (Lemmes 3-7) est celle de Wojdyslawski reconstruite et complétée de mémoire. 
puisque les ensembles $\{f(x)>c\}\{f(x)<c\}$ remplissent $M_{0}$, les points $a, b$ sont des points d'accumulation bilatéraux des ensembles $\{f(x)<c\},\{f(x)>c\}$ respectivement. On en déduit que les points de ces deux ensembles sont compris dans $(a, b)$ c'est-à-dire que $(a, b)\{f(x)>c\}$ et $(a, b)\{f(x)<c\}$ sont non vides. De cette manière nous obtenons la décomposition de l'intervalle $(a, b)$ ce qui est en contradiction avec le lemme 7. Il s'ensuit que $f(x) \in \mathcal{F}$, c'est-à-dire que

$$
\mathcal{X}_{0} \subset \mathcal{F} .
$$

D'autre part on peut voir aisément que $\mathcal{F} \subset \mathscr{C}_{1}$. Remarquons avant tout que si $f(x) \in \mathcal{F}$, les ensembles $\{f(x)>a\},\{f(x)<b\}$ sont du type $F_{\sigma}$ puisque $f(x)$ est de I classe de Baire. Si $\{f(x)>a\}$ n'est pas vide, il existe dans les intervalles $\left(x_{0}-\delta, x_{0}\right),\left(x_{0}, x_{0}+\delta\right)$ pour tout $x_{0}$ tel que $f\left(x_{0}\right)>a$ et pour chaque $\delta>0$ un ensemble ayant la puissance du continu des nombres $x$ tels que $f(x)$ $>a$. C'est évident si pour tout $x$ dans un certain intervalle $\left(x_{0}-\epsilon, x_{0}\right)$ ou $\left(x_{0}, x_{0}+\epsilon\right)$ on a $f(x)>a$. Si un tel nombre $\epsilon>0$ n'existe pas, il y a dans chaque intervalle $\left(x_{0}-\epsilon, x_{0}\right)$ évent. $\left(x_{0}, x_{0}+\epsilon\right)$ des valeurs $x_{\epsilon}$ telles que $f\left(x_{\epsilon}\right) \leqq a$. Puisque la fonction $f(x)$ jouit de la propriété de Darboux, elle prend dans $\left(x_{\epsilon}, x_{0}\right)$ un continu de valeurs intermédiaires entre $f\left(x_{\epsilon}\right)$ et $f\left(x_{0}\right)>a$, en particulier le continu de valeurs plus grandes que $a$. Il y a donc dans $\left(x_{0}-\epsilon, x_{0}\right)$ un ensemble de puissance du continu des valeurs de $x$ telles que $f(x)>a$, c'est-à-dire que $x_{0}$ est un point de condensation bilatéral de l'ensemble $\{f(x)>a\}$. La démonstration pour le cas $\{f(x)<b\}$ se fait d'une manière analogue, c'est-à-dire que $f(x) \in \mathscr{X}_{1}$, c.q.f.d. Nous avons donc en tenant compte de (5) et de la définition des classes $\mathcal{X}_{n}$ :

$$
\mathscr{X}_{1} \subset \mathscr{X}_{0} \subset \mathcal{F} \subset \mathscr{X}_{1} \subset \mathscr{X}_{0} \subset \mathcal{F}, \mathscr{X}_{0}=\mathscr{X}_{1}=\mathcal{F} .
$$

Le théorème suivante a pour but la généralisation du théorème élémentaire qui affirme que la fonction continue dont la dérivée est partout $\geqq 0$ est non décroissante. Je suppose que presque partout $f^{\prime}(x) \geqq 0$. On sait que sans autres suppositions ce théorème serait faux. Cependant MM. Goldowski [9], Tonelli [32] ont démontré que ce théorème est valable si nous supposons que la dérivée (finie ou infinie) existe partout. Le résultat le plus avancé dans cette direction fût obtenu par M. Tolstoff [31] qui démontre qu'une fonction approximativement continue ayant partout a l'exception d'un ensemble de points au plus dénombrable une dérivée approximative (finie ou infinie) presque partout non négative est une fonction non décroissante et continue. Dans le théorème que je démontrerai une supposition est plus forte que dans le théorème de $M$. Tolstoff et une autre supposition plus faible. Je ne suppose pas que $f(x)$ ait la propriété de Darboux mais je considère la dérivée ordinaire. Il est facile de construire des exemples qui montrent que cette supposition n'entraîne pas la validité du théorème pour la dérivée approximative. Par contre je pose le problème 
II. si le théorème de M. Tolstoff peut être généralisé pour les suppositions suivantes:

1. $f(x)$ est de I classe de Baire.

2. $f(x)$ a la proprièté de Darboux.

3. $f_{a p}^{\prime}(x)$ (évent. infinie) existe à l'exception d'un ensemble au plus dénombrable.

4. $f_{a p}^{\prime}(x) \geqq 0$ presque partout.

Il est facile de vérifier sur les exemples construits à l'aide de l'ensemble de Cantor (et même quand $f(x)$ est continue ou de classe $C_{1}$ sur complémentaire de cet ensemble) que la suppression même d'une seule de ces suppositions entrâ̂ne la fausseté du théorème. Je ne sais pas d'ailleurs, si l'on y peut remplacer $f_{a p}^{\prime}(x)$ par une définition plus faible de la dérivée.

La méthode employée ici pour la démonstration du Théorème 2 peut être appliquée aussi pour la preuve du théorème de $M$. Tolstoff.

Lemme $8\left(^{3}\right)$. Pour une fonction arbitraire finie $\left(^{4}\right) f(x)$ (même non mesurable $L$ ou dans l'autre théorie de mesure) l'ensemble $\left\{\bar{f}^{+}(x) \geqq a\right\}$ est du type $P+K_{0}$ ò̀ $K_{0}$ est une partie de l'ensemble $K$ de tous les points de discontinuité de $f(x)$ et $P$ un ensemble $G_{\delta}$ composé uniquement des points de continuité de la fonction $f(x)$.

Si l'ensemble $K$ est au plus dénombrable è $f(x)$ remplit la condition

$$
\begin{aligned}
& \liminf _{x \rightarrow x_{0}^{-}} f(x)<\limsup _{x \rightarrow x_{0}^{+}} f(x) \quad \text { ou } \\
& \liminf _{x \rightarrow x_{0}^{-}} f(x) \leqq f\left(x_{0}\right) \quad \text { si } \bar{f}^{+}\left(x_{0}\right)>-\infty, \text { ou } \\
& \limsup _{x \rightarrow x_{0}^{+}} f(x) \geqq f\left(x_{0}\right) \quad \text { si } \bar{f}^{-}\left(x_{0}\right)>-\infty,
\end{aligned}
$$

pour tout $x_{0}$ l'ensemble $\{\bar{f}(x) \geqq a\}$ est $G_{\delta}$.

En particulier $\{\bar{f}(x) \geqq a\}$ est $G_{\delta}$ si $f^{\prime}(x)$ (finie ou infinie) existe partout $\grave{a}$ l'exception d'un ensemble au plus dénombrable ( $f(x)$ pouvant être discontinue) et $f(x)$ remplit la condition de Darboux.

Démonstration. La première partie du lemme est évidemment vraie pour $a=-\infty$ car dans ce cas $\left\{\bar{f}^{+}(x) \geqq a\right\}=R$ (ensemble de tous les nombres réels finis) et $R=C+K$ où l'ensemble $C$ de tous les points de continuité de la fonction $f(x)$ est, comme on le sait, $G_{\delta}$. Pour $a=+\infty$ on a

(3) J'ai demontré le théorème concernant l'ensemble des points de la non-dérivabilité d'une fonction arbitraire en 1943; indépendamment de moi ce théorème a été démontré en 1941 (et publié en 1943) par M. A. Brudno [4]. Les deux démonstrations sont à quelques details près identiques, mais le travail de M. Brudno ne contenant pas la deuxième partie du théorème, j'expose ici toute la démonstration.

(4) En définissant d'une façon pour ainsi dire "non naturelle" la dérivée de Dini pour les $x$ tels que $f(x)=+\infty$ ou $-\infty$, nous pouvons supprimer cette supposition comme je la fais dans mon travail sur les ensembles des points de non-dérivabilité à publier prochainement. 


$$
\begin{aligned}
\left\{\bar{f}^{+}(x) \geqq a\right\}=\left\{\bar{f}^{+}(x)=+\infty\right\} & =\prod_{n=1}^{\infty}\left\{\bar{f}^{+}(x) \geqq n\right\}=\prod_{n=1}^{\infty}\left(P_{n}+K_{n}^{0}\right) \\
& =\prod_{n=1}^{\infty} P_{n}+K_{0}=P+K_{0} .
\end{aligned}
$$

La validité du lemme pour $a=+\infty$ résulte donc de sa validité pour $a$ fini. En considérant au lieu de $f(x)$ la fonction $f(x)-a x$, je ramène la question au cas de $a=0$. D'une manière analogue on peut ramener au cas de $a=0$ l'autre partie du lemme. On peut supposer sans restreindre la généralité que la fonction est bornée. Dans le cas contraire il suffit d'envisager au lieu de $f(x)$ la fonction

$$
\phi(x)=\frac{f(x)}{1+|f(x)|}
$$

pour laquelle l'ensemble de tous les points de discontinuité est le même que pour $f(x)$ et pour laquelle comme on le voit sańs difficulté

$$
\left\{\bar{\phi}^{+}(x) \geqq 0\right\}=\left\{\bar{f}^{+}(x) \geqq 0\right\} .
$$

Je suppose donc en plus que $|f(x)|<1$. Pour tout $x$ nous avons

$$
\begin{aligned}
\bar{f}^{+}(x) & =\lim _{n \rightarrow \infty} \lim _{m \rightarrow \infty} \phi_{m, n}(x), \\
\phi_{m, n}(x) & =\sup _{y \in I_{m, n}} \frac{f(y)-f(x)}{y-x}
\end{aligned}
$$

où $I_{m, n}=\left[x+a_{m, n}(x), x+a_{1, n}(x)\right]$, les intervalles $I_{m, n}$ pour $m \rightarrow \infty$ forment une suite croissante des ensembles et les intervalles (semi-ouverts) $\sum_{m=1}^{\infty} I_{m n}$ pour $n \rightarrow \infty$-une suite descendante (et la longueur $\rightarrow 0$ ). Il suffit pour cela que l'on ait

$$
\begin{gathered}
0<a_{m+1, n}(x) \leqq a_{m, n}(x), \quad a_{1, n+1}(x) \leqq a_{1, n}(x), \\
\lim _{m \rightarrow \infty} a_{m, n}(x)=0, \quad \lim _{n \rightarrow \infty} a_{1, n}(x)=0 .
\end{gathered}
$$

Les fonctions $a_{m, n}(x)$ ne doivent pas être nécessairement continues. Par un choix convenable de celles-ci (savoir tel que $x+a_{m, n}(x)$ ne prenne qu'un ensemble dénombrable de valeurs isolées) j'obtiens la démonstration du lemme. (En admettant que $a_{m, n}(x)$ soit indépendante de $x$, j'arrive au résultat plus faible exposé dans mon travail [34]. Le résultat exposé ici fut obtenu par moi en 1943).

Le Lemme 8 reste évidemment vrai aussi pour $\bar{f}^{-}(x)$; ainsi donc sa première partie le sera aussi pour $\bar{f}(x)=\max \left[\bar{f}^{+}(x), \bar{f}^{-}(x)\right]$ car $\{\bar{f}(x) \geqq a\}$ $=\left\{\bar{f}^{+}(x) \geqq a\right\}+\left\{\bar{f}^{-}(x) \geqq a\right\}$. Cependant, à cause de la deuxième partie du lemme, il faut définir directement $\bar{f}(x)$, comme on l'a fait dans le cas de 
$\bar{f}^{+}(x)$. On a

$$
\begin{aligned}
\bar{f}(x) & =\lim _{n \rightarrow \infty} \lim _{m \rightarrow \infty} \psi_{m, n}(x), \\
\psi_{m, n}(x) & =\sup _{y \in I_{m n+J_{m n}}} \frac{f(y)-f(x)}{y-x},
\end{aligned}
$$

où $J_{m n}=\left[x-b_{1, n}(x), x-b_{m, n}(x)\right]$ et les fonctions $b_{m, n}(x)$ remplissent les mêmes conditions (10), (11) que $a_{m, n}(x)$. Je pose

$$
\begin{aligned}
& x+a_{m, n}(x)=2^{-m-n} a \cdot\left(E\left[2^{m+n} a^{-1} x\right]+2\right), \\
& x-b_{m, n}(x)=2^{-m-n} a \cdot\left(E\left[2^{m+n} a^{-1} x\right]-1\right),
\end{aligned}
$$

où $E(\xi)$ est la partie entière du nombre $\xi$ et $a$ est une constante positive. Les fonctions $x+a_{m, n}(x), x-b_{m, n}(x)$ prennent un ensemble dénombrable de valeurs $2^{-m-n} k \cdot a, k=\cdots,-2,-1,0,1,2, \cdots$ Je désigne par $N=N_{a}$ l'ensemble de toutes les valeurs de toutes les fonctions $x+a_{m, n}(x), x-b_{m, n}(x)$. Il est facile de vérifier que les $a_{m, n}(x)$ et $b_{m, n}(x)$ remplissent les conditions (10), (11) ainsi que

$$
\begin{aligned}
& 2^{-m-n+1} a \geqq a_{m, n}(x) \geqq 2^{-m-n} a>0, \\
& 2^{-m-n+1} a \geqq b_{m, n}(x) \geqq 2^{-m-n} a .
\end{aligned}
$$

Il s'ensuit que

$$
\operatorname{dist}\left(x, I_{m n}\right) \geqq 2^{-m-n} a, \quad \operatorname{dist}\left(x, I_{m, n}+J_{m, n}\right) \geqq 2^{-m-n} a .
$$

Quand $x$ varie dans l'intervalle $\left(2^{-M-n} k \cdot a, 2^{-M-n} a \cdot(k+1)\right)$ les fonctions $x+a_{m n}(x), x-b_{m n}(x)$ restent constantes pour les $m \leqq M$ (et en particulier pour $m=1)$. En particulier pour $x \in L_{m n k}=\left(2^{-m-n} k a, 2^{-m-n}(k+1) a\right)$ les intervalles $I_{m n}, J_{m n}$ restent constants. Cela facilite l'étude de la continuité des fonctions $\phi_{m, n}(x), \psi_{m, n}(x)$. Soient $x_{0}$ et $x_{1}$ deux valeurs de l'intervalle $L_{m n k}$. Nous avons pour $y \in I_{m n}$ ou $I_{m n}+J_{m n}$

$$
\begin{aligned}
\left|\frac{f(y)-f\left(x_{1}\right)}{y-x_{1}}-\frac{f(y)-f\left(x_{0}\right)}{y-x_{0}}\right| \leqq & \frac{1}{\left|y-x_{1}\right| \cdot\left|y-x_{0}\right|} \mid f(y)\left(x_{1}-x_{0}\right) \\
& +y\left(f\left(x_{0}\right)-f\left(x_{1}\right)\right)+x_{0} f\left(x_{1}\right)-x_{1} f\left(x_{0}\right) \mid \\
\leqq & 2^{2 m+2 n} a^{-2}\left(\left|x_{1}-x_{0}\right|+|y|\left|f\left(x_{1}\right)-f\left(x_{0}\right)\right|\right. \\
& +\left|x_{0}+x_{1}\right|\left|f\left(x_{1}\right)-f\left(x_{0}\right)\right| \\
& \left.+\left|x_{0} f\left(x_{0}\right)-x_{1} f\left(x_{1}\right)\right|\right)
\end{aligned}
$$

puisque $|f(y)|<1$ et $\left|y-x_{1}\right| \geqq \operatorname{dist}\left(x_{1}, I_{m n}\right)$ évent. dist $\left(x_{1}, I_{m n}+J_{m n}\right)$; donc d'après (14) $\left|y-x_{1}\right| \geqq 2^{-m-n} a$, pareillement $\left|y-x_{0}\right| \geqq 2^{-m-n} a$. Puisque $|y|$, $\left|x_{0}+x_{1}\right|$ ont une borne supérieure commune $=a \cdot 2^{-m-n} \cdot 2\left(|k|+2^{m}\right)$ pour $y \in I_{m, n}+J_{m, n}$, et $\left[x_{0}\right]+\left[x_{1}\right] \subset L_{m n k}$, donc pour les $\left|x_{1}-x_{0}\right|,\left|f\left(x_{1}\right)-f\left(x_{0}\right)\right|$, $\left|x_{1} f\left(x_{1}\right)-x_{0} f\left(x_{0}\right)\right|$ suffisamment petits nous obtenons 


$$
\left|\frac{f(y)-f\left(x_{1}\right)}{y-x_{1}}-\frac{f(y)-f\left(x_{0}\right)}{y-x_{0}}\right|<\epsilon \text { pour tout } y \in I_{m n}+J_{m n} .
$$

En particulier il résulte de l'uniformité de cette évaluation et de (9), (13) si $x_{0}$ est un point de continuité de la fonction $f(x)$ que:

$$
\left|\phi_{m, n}\left(x_{0}\right)-\phi_{m, n}(x)\right|<\epsilon,\left|\psi_{m, n}\left(x_{0}\right)-\psi_{m, n}(x)\right|<\epsilon \text { pour }\left|x-x_{0}\right|<\delta,
$$

c'est-à-dire que les fonctions $\phi_{m n}(x), \psi_{m n}(x)$ sont continues aux points de continuité de la fonction $f(x)$ situés dans $L_{m n k}$. Les discontinuités éventuelles ne peuvent donc paraitre qu'aux points de l'ensemble $K$ et aux points $2^{-m-n} k a, k=\cdots,-2,-1,0,1,2, \cdots$, ce qui veut dire que l'ensemble de tous les points de discontinuité des fonctions $\phi_{m n}(x), \psi_{m n}(x)$ est contenu dans $K+N$. Les fonctions $a_{m n}(x), b_{m n}(x)$ étant ainsi choisies, il résulte de (10) que les passages à la limite dans (8), (12) sont monotoniques. Il s'ensuit que

$$
\begin{gathered}
\left\{\bar{f}^{+}(x)>-\frac{1}{k}\right\} \subset \prod_{n=1}^{\infty}\left\{\lim _{m \rightarrow \infty} \phi_{m n}(x)>-\frac{1}{k}\right\} \subset\left\{\bar{f}^{+}(x) \geqq-\frac{1}{k}\right\}, \\
\left\{\lim _{m \rightarrow \infty} \phi_{m n}(x)>-\frac{1}{k}\right\}=\sum_{m=1}^{\infty}\left\{\phi_{m n}(x)>-\frac{1}{k}\right\}
\end{gathered}
$$

et puisque

$$
\left\{\bar{f}^{+}(x) \geqq 0\right\}=\prod_{k=1}^{\infty}\left\{\bar{f}^{+}(x)>-\frac{1}{k}\right\}=\prod_{k=1}^{\infty}\left\{\bar{f}^{+}(x) \geqq-\frac{1}{k}\right\},
$$

on aura

$$
\left\{\bar{f}^{+}(x) \geqq 0\right\}=\prod_{k=1}^{\infty} \prod_{n=1}^{\infty} \sum_{m=1}^{\infty}\left\{\phi_{m, n}(x)>-\frac{1}{k}\right\} .
$$

Si le point $x_{0}$ de l'ensemble $\left\{\phi_{m n}(x)>-1 / k\right\}$ est un point de continuité de la fonction $\phi_{m n}(x)$, un certain voisinage de ce point appartient également à cet ensemble, donc $x_{0}$ est un point intérieur de l'ensemble $\left\{\phi_{m n}(x)>-1 / k\right\}$. Il en résulte que l'ensemble $\left\{\phi_{m n}(x)>-1 / k\right\}-$ int $\left\{\phi_{m n}(x)>-1 / k\right\}$ se compose des points de discontinuité de la fonction $\phi_{m n}(x)$. Il se compose donc de deux parties: $K_{m n k} \subset K$ et $N_{m n k} \subset N$. En posant int $\left\{\phi_{m n}(x)>-1 / k\right\}=G_{m n k}$ on a donc

$$
\begin{aligned}
\left\{\bar{f}^{+}(x) \geqq 0\right\} & =\prod_{k=1}^{\infty} \prod_{n=1}^{\infty} \sum_{m=1}^{\infty}\left(G_{m n k}+K_{m n k}+N_{m n k}\right) \\
& =\prod_{k=1}^{\infty} \prod_{n=1}^{\infty}\left(G_{n k}+K_{n k}+N_{n k}\right) \\
& =\prod_{k=1}^{\infty} \prod_{n=1}^{\infty} G_{n k}+K_{0}+N_{0}=P+K_{0}+N_{0}
\end{aligned}
$$


où les ensembles $G_{m n k}, G_{n k}$ sont ouverts, $K_{0} \subset \sum_{k=1}^{\infty} \sum_{n=1}^{\infty} \sum_{m=1}^{\infty} K_{m n k} \subset K$, $N_{0} \subset N$, donc l'ensemble $P=\prod_{k=1}^{\infty} \prod_{n=1}^{\infty} G_{n k}$ est du type $G_{\delta}$. La formule ainsi obtenue (15) dépend de la constante $a$ qui détermine l'ensemble $N=N_{a}$. Considérons deux pareilles représentations de l'ensemble $\left\{\bar{f}^{+}(x) \geqq 0\right\}$; une pour $a=1$ et l'autre pour $a=2^{1 / 2}$; pour simplifier l'écriture je désignerai dans le second cas $N_{a}$ par $N_{2}$. Je distingue les décompositions de l'ensemble $\left\{\bar{f}^{+}(x) \geqq 0\right\}$ dans les I et II cas d'après la formule (15) à l'aide des indices additionnels. L'ensemble $N_{1}$ ne renferme que les nombres rationnels et $N_{2}$ renferme un seul nombre rationnel (=0), donc $N_{1} N_{2}=$ [0]. De (15) j'obtiens:

$$
\begin{aligned}
\left\{\bar{f}^{+}(x) \geqq 0\right\} & =P_{1}+K_{10}+N_{10}=P_{2}+K_{20}+N_{20} \\
& =\left(P_{1}+K_{10}+N_{10}\right)\left(P_{2}+K_{20}+N_{20}\right)+P_{1}+P_{2} \\
& =P_{1}+P_{2}+K_{10}\left(K_{20}+N_{20}\right)+K_{20} N_{10}+N_{10} N_{20} \\
& =\left(P_{1}+P_{2}+N_{10} N_{20}\right)+K_{.0},
\end{aligned}
$$

où $K .0$, $\subset$, et $N_{10} N_{20} \subset N_{1} N_{2}=$ [0] est l'ensemble qui ne se compose que d'un seul point [0] ou est vide, donc est $G_{\delta}$. Il s'ensuit que $P .=P_{1}+P_{2}+N_{10} N_{20}$ étant une somme de trois ensembles $G_{\delta}$ est aussi $G_{\delta}$. Puisque

$$
\begin{aligned}
\left\{\bar{f}^{+}(x) \geqq 0\right\} & =\left\{\bar{f}^{+}(x) \geqq 0\right\} R=\left(P .+K_{.0}\right)(C+K) \\
& =C P .+K P .+K_{.0}(C+K)=P+K_{0},
\end{aligned}
$$

où $P=C P . \subset C$ est $G_{\delta}$ car $C$ qui est l'ensemble de tous les points de continuité de la fonction $f(x)$ est comme on sait, $G_{\delta}$. La première partie du lemme est ainsi prouvée. D'une manière tout à fait analogue en considérant $\psi_{m n}(x)$ au lieu de $\phi_{m n}(x)$, j'obtiens le même résultat pour $\{\bar{f}(x) \geqq 0\}$ et en particulier la formule analogue à (15), (16). Pour simplifier je garde les mêmes notations $\left(G_{n k}\right.$ etc.) ce qui ne prêtera à aucune confusion. Nous avons donc

$$
\{\bar{f}(x) \geqq 0\}=\prod_{k=1}^{\infty} \prod_{n=1}^{\infty}\left(G_{n k}+K_{n k}+N_{n k}\right)=P+K_{0},
$$

où $G_{n k}$ est un ensemble ouvert, $K_{n k} \subset K, N_{n k} \subset N, P \subset C, K_{0} \subset K, P$ est du type $G_{\delta}$. De là

$$
\begin{aligned}
P+K_{0} & \subset G_{n k}+K_{n k}+N_{n k}, \\
G_{n k}+K_{n k}+N_{n k} & =G_{n k}+K_{n k}+N_{n k}+P+K_{0} \quad \text { pour tous les } n, k, \\
P+K_{0} & =\prod_{k=1}^{\infty} \prod_{n=1}^{\infty}\left(P+K_{0}\right)\left(G_{n k}+K_{n k}+N_{n k}+P+K_{0}\right) \\
& \subset \prod_{k=1}^{\infty} \prod_{n=1}^{\infty}\left(P+K_{0}+G_{n k}+K_{0} K_{n k}+P N_{n k}+K_{0} N_{n k}\right) \\
& =\prod_{k=1}^{\infty} \prod_{n=1}^{\infty}\left(P+K_{0}+G_{n k}\right)=\prod_{k=1}^{\infty} \prod_{n=1}^{\infty}\left(P+K_{n k}^{*}+G_{n k}\right),
\end{aligned}
$$


où $K_{n k}^{*}=K_{0}-G_{n k}$. De (17) résulte par conséquent

$$
\begin{aligned}
\bar{f}(x) & \geqq 0 \text { pour tout } x \in K_{n k}^{*}, \\
K_{n k}^{*} G_{n k} & =0 .
\end{aligned}
$$

D'autre part

$$
\begin{aligned}
P+K_{n k}^{*}+G_{n k} & =P+K_{0}+G_{n k} \subset P+K_{0}+G_{n k}+K_{n k}+N_{n k} \\
& =G_{n k}+K_{n k}+N_{n k},
\end{aligned}
$$

donc il s'ensuit de (17) que

$$
\prod_{k=1}^{\infty} \prod_{n=1}^{\infty}\left(P+K_{n k}^{*}+G_{n k}\right) \subset \prod_{k=1}^{\infty} \prod_{n=1}^{\infty}\left(G_{n k}+K_{n k}+N_{n k}\right)=P+K_{0}
$$

De cela et de (18) résulte

$$
\{\bar{f}(x) \geqq 0\}=P+K_{0}=\prod_{k=1}^{\infty} \prod_{n=1}^{\infty}\left(P+K_{n k}^{*}+G_{n k}\right) .
$$

Je vais démontrer que l'ensemble $K_{n k}^{*}$, au plus dénombrable (car $K_{n k}^{*} \subset K$ ), est du type $G_{\delta}$. $P$ étant $G_{\delta}$, il en est de même des facteurs du second membre de la formule (21) qui se composent d'une somme de deux ensembles $G_{\delta}$ et d'un ensemble ouvert, donc $\{\bar{f}(x) \geqq 0\}$ est $G_{\delta}$, c.q.f.d. Il reste à montrer que $K_{n k}^{*}$ est $G_{\delta} . K_{n k}^{*}$ étant au plus dénombrable, il faut et il suffit de prouver, d'après les propositions connues, que $K_{n k}^{*}$ ne contient pas une partie dense en soi. Supposons que le contraire soit vrai, c'est-à-dire que $K_{n k}^{*}$ renferme une partie $Z$ dense en soi. Dans ce cas l'ensemble parfait $\bar{Z}$ contiendrait $Z$ et $Z$ serait dense sur $\bar{Z}$ (les points de l'ensemble $Z$ sont situés à l'intérieur de chaque segment de l'ensemble $\bar{Z}$ ). D'autre part de ce que $Z \subset K_{n k}^{*}$ et de (20) résulte

$$
G_{n k} \bar{Z}=0 .
$$

De (19) s'ensuit

$$
\bar{f}(x) \geqq 0 \text { pour tout } x \in Z,
$$

on peut donc faire correspondre à chaque $\epsilon$ un segment $[a, b]$ arbitrairement court renfermant $x$ à son intérieur et tel que

$$
\frac{f(b)-f(a)}{b-a}>-\epsilon \text {. }
$$

C'est évident si $\lim \inf _{t \rightarrow x^{-}} f(t)<\lim \sup _{t \rightarrow x^{+}} f(t)$. Dans le cas contraire, puisque on a p.ex. $\bar{f}^{+}(x) \geqq 0$, il résulte de $(6)$

$$
\liminf _{t \rightarrow x^{-}} f(t) \leqq f(x) .
$$


On peut faire correspondre à chaque $\eta>0$ un nombre $b>x$, tel que

$$
b-x<\frac{\eta}{2}, \quad \frac{f(b)-f(x)}{b-x}>-\frac{\epsilon}{2} .
$$

D'après (24) il existe un nombre $a<x$, tel que

$$
\begin{gathered}
x-a<\frac{\eta}{2}, \quad(x-a)|f(x)-f(b)|<\frac{\epsilon}{4}(b-x)^{2}, \\
f(a)<f(x)+\frac{\epsilon}{4}(b-x),
\end{gathered}
$$

de là

$$
\begin{gathered}
(x-a)(f(x)-f(b))>-\frac{\epsilon}{4}(b-x)^{2}>-\frac{\epsilon}{4}-(b-a)(b-x), \\
(b-x)(f(x)-f(a))>-\frac{\epsilon}{4}(b-x)^{2}>-\frac{\epsilon}{4}(b-a)(b-x), \\
a f(b)-x f(b)-a f(x)+b f(x)-b f(a)+x f(a)>-\frac{\epsilon}{2}(b-a)(b-x), \\
\frac{f(b)-f(a)}{b-a}-\frac{f(b)-f(x)}{b-x}>-\frac{\epsilon}{2}
\end{gathered}
$$

et de (25), (26) résulte (23) avec $b-a<\eta$. Pour $\bar{f}-(x) \geqq 0$ la démonstration est analogue.

Je forme la suite descendante des segments de l'ensemble $\bar{Z},\left[a_{n}, b_{n}\right] \bar{Z}$, où $\lim _{n \rightarrow \infty}\left(b_{n}-a_{n}\right)=0$. D'après le théorème de Cantor le produit de ces segments est le point $\xi \in \bar{Z}$. Je vais choisir les intervalles $\left[a_{n}, b_{n}\right]$ de telle manière qu'ils remplissent la condition (23) avec $\epsilon=n^{-1}$ en évitant les points de l'ensemble dénombrable $K+N$. En désignant par $x_{1}, x_{2}, \cdots$ la suite de tous les points de l'ensemble $K+N$ (renfermant en particulier tous les points de l'ensemble $Z \subset K_{n k}^{*} \subset K$ ), je choisis un point $x_{(1)} \in Z, x_{(1)} \neq x_{1}$ (ce qui est possible puisque $Z$ renferme une infinité des points) et je forme l'intervalle $\left[a_{1}, b_{1}\right]$ renfermant à son intérieur $x_{(1)}$ ne renfermant pas $x_{1}$ et tel que

$$
f\left(b_{1}\right)-f\left(a_{1}\right)>-1\left(b_{1}-a_{1}\right), \quad \cdot b_{1}-a_{1}<1 .
$$

On aura donc à l'intérieur de l'intervalle $\left[a_{1}, b_{1}\right]$ une infinité des points de l'ensemble $Z$ (puisque $Z$ est dense en soi donc $x_{(1)}$ est un point d'accumulation de l'ensemble $\left.Z, a_{1}<x_{(1)}<b_{1}\right)$. Je définis $\left[a_{k}, b_{k}\right]$ par récurrence. Si l'on a défini déjà les intervalles $\left[a_{1}, b_{1}\right], \cdots,\left[a_{n}, b_{n}\right]$ remplissant les conditions:

$$
f\left(b_{k}\right)-f\left(a_{k}\right)>-\frac{1}{k}\left(b_{k}-a_{k}\right), \quad k=1,2, \cdots, n,
$$




$$
\begin{array}{rlr}
x_{k} & \oplus\left[a_{k}, b_{k}\right], & k=1,2, \cdots, n, \\
{\left[a_{k+1}, b_{k+1}\right]} & \subset\left(a_{k}, b_{k}\right), & k=1,2,3, \cdots, n-1, \\
b_{k}-a_{k}<\frac{1}{k}, & k=1,2, \cdots, n,
\end{array}
$$

et renfermant à l'intérieur une infinité des points de l'ensemble $Z$, je définis l'intervalle $\left[a_{n+1}, b_{n+1}\right]$ de la manière suivante: je choisis le point $x_{(n+1)}$ $\in Z\left(a_{n}, b_{n}\right), x_{(n+1)} \neq x_{n+1}$ et je forme l'intervalle $\left[a_{n+1}, b_{n+1}\right], a_{n+1}<x_{(n+1)}<b_{n+1}$ assez petit pour qu'il soit situé à l'intérieur de $\left[a_{n}, b_{n}\right], b_{n+1}-a_{n+1}<1 /(n+1)$, ne contienne pas $x_{n+1}$ et $f\left(b_{n+1}\right)-f\left(a_{n+1}\right)>-(1 /(n+1))\left(b_{n+1}-a_{n+1}\right)$. L'intervalle $\left(a_{n+1}, b_{n+1}\right)$ contenant $x_{(n+1)} \in Z$ renferme une infinité des points de l'ensemble $Z$ (donc ces points appartient à l'intérieur de $\left[a_{n+1}, b_{n+1}\right]$ ). La construction de $\left[a_{k}, b_{k}\right]$ est donc possible pour tout $k$ et les conditions (27)(30) sont remplies pour chaque $k$ entier, positif, en particulier tous les segments $\left[a_{k}, b_{k}\right] \bar{Z}$ de l'ensemble $\bar{Z}$ sont non vides. Il résulte de (28) que

$$
\lim _{n \rightarrow \infty} a_{n}=\xi \neq x_{k} \text { pour tout } \quad k, \xi \notin K+N \text {. }
$$

De là et de (22) en tenant compte de ce que $\xi \in \bar{Z}$ :

$$
\xi \in \prod_{k=1}^{\infty} \prod_{n=1}^{\infty} G_{n k}+N+K \supset \prod_{k=1}^{\infty} \prod_{n=1}^{\infty}\left(G_{n k}+K_{n k}+N_{n k}\right)=\{\bar{f}(x) \geqq 0\},
$$

d'après la formule (17), on a donc

$$
\bar{f}(\xi)<0 .
$$

D'autre part il résulte de (27) que

$$
\bar{f}(\xi) \geqq 0 .
$$

Il s'ensuit de cette contradiction que $K_{n k}^{*}$ ne contient pas une partie dense en soi, c.q.f.d.

En particulier si $f(x)$ a une dérivée sauf aux points d'un ensemble $E$ au plus dénombrable, $f(x)$ ne peut être discontinue qu'aux points de non-dérivabilité, ou aux points où $\left|f^{\prime}(x)\right|=+\infty$. L'ensemble des premiers points est d'après la supposition au plus dénombrable; l'ensemble des seconds-est au plus dénombrable d'après une proposition connue de Young sur la symétrie, puisqu'au point de discontinuité en lequel existe la dérivée infinie les ensembles des limites à droite et à gauche ne peuvent pas être identiques. La condition (6) est remplie, car si $f(x)$ a la propriété de Darboux, alors $\lim \inf _{x \rightarrow x_{0}^{-}} f(x) \leqq f\left(x_{0}\right) \leqq \lim \sup _{x \rightarrow x_{0}^{-}} f(x), \lim \inf _{x \rightarrow x_{0}^{+}} f(x) \leqq f\left(x_{0}\right) \leqq \lim \sup _{x \rightarrow x_{0}^{+}}$ $f(x)$. Il en découle d'après la II partie du lemme déjà démontré que $\{\bar{f}(x) \geqq 0\}$ est du type $G_{\delta}$, c.q.f.d.

Corollaire. La première dêrivée $f^{\prime}(x)$ est de I classe de Baire (même pour 
$f(x)$ discontinue).

Si $f^{\prime}(x)$ existe partout, $K$ est au plus dénombrable et $f(x)$ satisfait évidemment la condition (6), donc $\left\{f^{\prime}(x) \geqq a\right\}$ est $G_{\delta}$ pour tout $a$, pareillement $\left\{f^{\prime}(x) \leqq b\right\}$ c-à-d. $\left\{-f^{\prime}(x) \geqq-b\right\}$ pour tout $b$, c.q.f.d. (Ce corollaire résulte aussi d'un travail de M. Kronrod [16].)

Pour la fonction arbitraire on peut montrer que $\{\bar{f}(x) \geqq a\}=P+(K-B)$, où $P \in G_{\delta}, B$ est au plus dénombrable et $\{\bar{f}(x) \geqq a\}=P_{1}+\left(K_{1}-B_{1}\right)$ où $P_{1} \in G_{\delta}$, $K_{1} \subset K, K_{1} \in F_{\sigma},\left|K_{1}\right|=0, B_{1}$ au plus dénombrable. Si l'ensemble $K$ est dénombrable mais $f(x)$ ne satisfait pas $(6),\{\bar{f}(x) \geqq a\}$ n'est pas en général $G_{\delta}$. Je laisse au lecteur de former des contre-exemples faciles (avec ensemble du type de Cantor).

Leмme 9. (Théorème de Rolle sur la valeur moyenne.) Si $f(a)=f(b)$ et la fonction $f(x)$ a la propriêté de Darboux, il existe un point $\xi \in(a, b)$ tel que

$$
\bar{f}(\xi) \geqq 0, \quad f(\xi) \leqq 0 .
$$

Démonstration. On peut exclure le cas où il existe un segment $(c, d) \subset(a, b)$ tel que $f(c)=f(d)$ et $f(x)$ est continue dans $[c, d]$ car dans ce cas le lemme résulte du théorème élémentaire de Rolle. En particulier il n'y a pas d'intervalle où la fonction $f(x)$ serait constante. Si $[c, d]$ renferme un point de discontinuité de $f(x)$ par rapport a $[c, d]$, il existe une valeur $u$ telle que l'équation $f(x)=u$ possède une infinité de solutions dans $(c, d)$. En effet, en un certain point $x_{0}$ il existe une valeur limite $\theta$ de la fonction $f(x)$ différente de $f\left(x_{0}\right)$, p.ex. $f\left(x_{0}\right)>\theta$. Il existe donc une suite de points $x_{n} \in(c, d), x_{n} \rightarrow x_{0}$ tels que $f\left(x_{n}\right) \rightarrow \theta$ et l'on peut de suite supposer que $f\left(x_{n}\right)<\left(f\left(x_{0}\right)+\theta\right) / 2$ (laissant éventuellement de côté un nombre fini des points). On peut choisir $x_{n}$ d'un côté de $x_{0}$. Supposons p.ex. que $x_{n}<x_{0}$ (le second cas est analogue). Dans chaque intervalle $\left(x_{n}, x_{0}\right)$ la fonction $f(x)$ prend la valeur intermédiaire $u=\left(f\left(x_{0}\right)+\theta\right) / 2$; elle la prend donc une infinité de fois. En me basant sur cela je vais définir une suite descendante d'intervalles fermés dont le produit sera le point $\xi$, leurs longueurs tendront donc vers zéro. Le premier de ces intervalles $\left[a_{1}, b_{1}\right]$ sera l'intervalle $[a, b]$. D'après la supposition complémentaire $[a, b]$ renferme un point de discontinuité de $f(x)$ par rapport a $[a, b]$; il existe donc une valeur $u_{1}$ telle que l'équation $f(x)=u_{1}$ possède une infinité de solutions dans $(a, b)$. Je choisis trois d'entre elles: $x_{1}<x_{2}<x_{3}$. Puisque $x_{3}-x_{1}<b-a$ et $\min \left(x_{2}-x_{1}, x_{3}-x_{2}\right) \leqq 2^{-1}\left(x_{3}-x_{1}\right)$, nous pouvons choisir l'intervalle $\left[a_{2}, b_{2}\right]=\left[x_{1}, x_{2}\right]$ ou $\left[x_{2}, x_{3}\right]$ de manière que $b_{2}-a_{2}<2^{-1}(b-a)$. On a donc $f\left(b_{2}\right)=f\left(a_{2}\right)$ et l'intervalle $\left[a_{2}, b_{2}\right]$ renferme d'après la même supposition complémentaire un point de discontinuité de $f(x)$. Supposons que l'on ait déjà défini l'intervalle $\left[a_{n}, b_{n}\right] \subset\left(a_{n-1}, b_{n-1}\right)$ tel que

$$
f\left(b_{n}\right)=f\left(a_{n}\right), \quad b_{n}-a_{n}<2^{-n+1}(b-a) .
$$

D'après la supposition complémentaire $\left[a_{n}, b_{n}\right]$ renferme un point de dis- 
continuité de la fonction $f(x)$ par rapport à $\left[a_{n}, b_{n}\right]$; on peut donc comme dans le cas de $(a, b)$, trouver trois points $x_{1}^{\prime}<x_{2}^{\prime}<x_{3}^{\prime}$ dans $\left(a_{n}, b_{n}\right)$ tels que $f\left(x_{1}^{\prime}\right)=f\left(x_{2}^{\prime}\right)=f\left(x_{3}^{\prime}\right)$; je choisis pour $\left[a_{n+1}, b_{n+1}\right]$ le plus petit de deux intervalles $\left[x_{1}^{\prime}, x_{2}^{\prime}\right],\left[x_{2}^{\prime}, x_{3}^{\prime}\right]$. Dans ce cas $b_{n+1}-a_{n+1}<2^{-1}\left(b_{n}-a_{n}\right)<2^{-n}(b-a)$. Les conditions (31) sont donc remplies pour $n+1$ en place de $n$ et les nombres $a_{n}, b_{n}$ sont déterminés par induction pour chaque $n$. De (31) résulte

$$
\bar{f}(\xi) \geqq 0, \quad f(\xi) \leqq 0 \quad \text { pour } \quad \xi=\lim _{n \rightarrow \infty} a_{n}, \quad a_{n}<\xi<b_{n}, \text { c.q.f.d. }
$$

Corollaire. (Propriêté de Darboux de la dérivée.) Si une dérivée intermédiaire unilatèrale $f_{p}^{\prime}(x)$ de la fonction $f(x)$ de classe $\mathcal{F}$ remplit les inégalités

$$
f_{p}^{+}(a)<c, \quad \overrightarrow{f_{p}}(b)>c \quad \text { ou } \quad f_{p}^{+}(a)>c, \quad \overrightarrow{f_{p}}(b)<c,
$$

il existe un point $\xi \in(a, b)$ tel que $f(\xi) \leqq c, \bar{f}(\xi) \geqq c$.

( $J^{\prime} a p p e l l e$ dérivée intermédiairé chaque fonction $f_{p}^{+}(x)$ remplissant partout l'inégalité $\left.\bar{f}^{+}(x) \geqq f_{p}^{+}(x) \geqq \underline{f}^{+}(x)\right)$.

En considérant au lieu de $f(x)$ la fonction $f(x)-c x$ je ramène le cas général au cas de $c=0$. La fonction $f(x)-c x$ est aussi de classe $\mathcal{F}$. En effet, elle est de I classe de Baire comme la difference des deux fonctions de I classe, donc les ensembles $\{f(x)-c x>a\}$ sont du type $F_{\sigma}$. Mais

$$
\begin{aligned}
\{f(x)-c x>a\} & =\{f(x)>c x+a\} \\
& =\sum_{m, n}\left\{f(x)>\frac{m+1}{n}>\frac{m}{n}>c x+a\right\} \\
& =\sum_{m, n}\left\{f(x)>\frac{m+1}{n}\right\}\left\{c x+a<\frac{m}{n}\right\} .
\end{aligned}
$$

Les ensembles $\{c x+a<m / n\}$ sont ouverts et $\{f(x)>(m+1) / n\}$ sont de classe $M_{1}$, donc, en vertu du Lemme 1 et Lemme $2,\{f(x)-c x>a\}$ est de classe $M_{1}$. La preuve pour $\{f(x)-c x<a\}$ est analogue. Il en résulte que $f(x)-c x \in \mathscr{X}_{1}=\mathcal{F}$ d'après le Théorème 1 , possède donc la propriété de Darboux. (La propriété de Darboux, en dehors de la classe $\mathcal{F}$, n'est pas conservée en général après l'addition d'une fonction linéaire.)

Je prends en considération le premier couple d'inégalités; pour le second la démonstration est analogue. Il résulte de celles-ci que $f(x)$ prend dans le voisinage à droite du point $a$ les valeurs inférieures à $f(a)$ et dans le voisinage à gauche de $b$-les valeurs $<f(b)$. Si $f(a)=f(b)$ le corollaire résulte immédiatement du Lemme 9. Supposons que p.ex. $f(a)>f(b)$ (dans le cas contraire la démonstration est pareille). Il existe dans $(a, b)$ un point $d$ tel que $f(d)<f(b)$, donc il y a dans $(a, d)$ un point $e$ en lequel $f(x)$ prend la valeur intermédiaire $f(b)$; on a donc $(e, b) \subset(a, b), f(e)=f(b)$ et d'après le Lemme 9 il existe un point $\xi \in(e, b)$ donc $\xi \in(a, b)$ ayant la propriété désirée. 
LEMME 10. Si une fonction finie $f(x)$ n'est pas non décroissante dans $[a, b]$ et si l'on a presque partout $\vec{f}(x) \geqq 0$ dans $[a, b]$, il existe dans $[a, b]$ un point $\xi$ tel que $\underline{f}(\xi)=-\infty$.

Démonstration. Supposons que tous les quotients des différences dans $[a, b]$ soient bornés en bas

$$
\frac{f\left(x_{1}\right)-f\left(x_{2}\right)}{x_{1}-x_{2}} \geqq-M, \quad 0<M<+\infty .
$$

La fonction $f_{1}(x)=f(x)+2 M x$ est croissante dans $[a, b]$, donc presque partout dérivable. En soustrayant de $f_{1}(x)$ sa fonction de discontinuités, on obtient une fonction continue non décroissante $\phi(x)$ telle que pour $x_{1}<x_{2}$ on a

$$
\phi\left(x_{2}\right)-\phi\left(x_{1}\right) \leqq f\left(x_{2}\right)-f\left(x_{1}\right)+2 M\left(x_{2}-x_{1}\right) .
$$

[On appelle fonction de discontinuités d'une fonction monotone $F(x)$ la somme $\sum_{n} \omega_{n}(x)$ où la fonction $\omega_{n}(x)$ correspond au point de discontinuité $x_{n}$ de $F(x)$. (La suite des $x_{n}$ étant au plus dénombrable.) $\mathrm{Si}$

$$
\lim _{x \rightarrow x_{n}^{-}} F(x)=a_{n} \leqq F\left(x_{n}\right) \leqq b_{n}=\lim _{x \rightarrow x_{n}^{+}} F(x),
$$

alors

$$
\omega_{n}(x)=\left\{\begin{array}{lll}
0 & \text { pour tout } & x<x_{n}, \\
F\left(x_{n}\right)-a_{n} & \text { pour } & x=x_{n} \\
b_{n}-a_{n} & \text { pour tout } & x>x_{n}
\end{array}\right.
$$

La convergence uniforme de la série $\sum_{n} \omega_{n}(x)$ et les propriétés utilisées ici sont manifestes.]

Puisque $\underline{\phi}(x) \geqq 0$, on a pour une fonction continue $\psi(x)=\phi(x)-2 M x$ :

$$
\underline{\psi}(x) \geqq-2 M \quad \text { pour tous les } x .
$$

La dérivée de la fonction de discontinuités étant presque partout égale a 0 , on a

$$
\begin{aligned}
& \phi^{\prime}(x)=f^{\prime}(x)+2 M \\
& \psi^{\prime}(x)=\phi^{\prime}(x)-2 M=f^{\prime}(x) \geqq 0 \quad \text { presque partout. }
\end{aligned}
$$

Il s'ensuit à cause de (33), que la fonction continue $\psi(x)$ est en vertu des propositions connues non décroissante dans $[a, b]$. On a notamment pour tout $\epsilon>0$ et $\psi_{\epsilon}(x)=\psi(x)+\epsilon x: \psi_{\epsilon}^{\prime}(x)>0$ presque partout, $\underline{\psi}_{\epsilon}(x)>-2 M$ partout, donc $\left|\Psi_{\epsilon}^{+}(x)\right|<2 M$ pour tout $x \in\left\{\psi_{\epsilon}^{+}(x) \leqq 0\right\}$. Il résulte (Saks [27, p. 271 , Théorème (4.5)]) que $\left|\psi_{\epsilon}\left(\left\{\Psi_{\epsilon}^{+}(x) \leqq 0\right\}\right)\right| \leqq\left|\left\{\Psi_{\epsilon}^{+}(x) \leqq 0\right\}\right| \cdot 2 M=0$, car $\left|\left\{\Psi_{\epsilon}^{+}(x) \leqq 0\right\}\right|=0$. D'après Théorème (7.1) de M. A. Zygmund [27, p. 203], $\psi_{\epsilon}(x)$ est non décroissante. Il résulte de (32) que 


$$
f\left(x_{2}\right)-f\left(x_{1}\right) \geqq \psi\left(x_{2}\right)-\psi\left(x_{1}\right) .
$$

La fonction $f(x)$ serait donc aussi non décroissante ce qui est en contradiction avec la supposition.

Il s'ensuit que pour chaque nombre $M$ existent dans $[a, b]$ les points $x_{1}, x_{2}$ tels que

$$
\frac{f\left(x_{2}\right)-f\left(x_{1}\right)}{x_{2}-x_{1}}<-M .
$$

En bassant sur cela, je vais former une suite descendante d'intervalles $\left[a_{n}, b_{n}\right]$, $b_{n}-a_{n} \rightarrow 0$ dont le produit est le point cherché $\xi$. Sans restreindre la généralité je peux supposer que la fonction $f(x)$ soit bornée, $|f(x)|<1$. (Comme dans le Lemme 8 , (7).) Je choisis $\left[a_{1}, b_{1}\right]$ tel que

$$
\frac{f\left(b_{1}\right)-f\left(a_{1}\right)}{b_{1}-a_{1}}<-1 .
$$

Supposons que l'on ait déjà déterminé l'intervalle $\left[a_{n}, b_{n}\right] \subset\left[a_{n-1}, b_{n-1}\right]$ tel que

$$
\frac{f\left(b_{n}\right)-f\left(a_{n}\right)}{b_{n}-a_{n}}<-n .
$$

La fonction $f(x)$ n'étant pas non décroissante dans $\left[a_{n}, b_{n}\right]$, il y a dans $\left[a_{n}, b_{n}\right]$ des points $a_{n+1}, b_{n+1}$ tels que

$$
\frac{f\left(b_{n+1}\right)-f\left(a_{n+1}\right)}{b_{n+1}-a_{n+1}}<-n-1 .
$$

De cette manière la suite d'intervalles $\left[a_{n}, b_{n}\right]$ est déterminée par induction. Il résulte de (34) que

$$
b_{n}-a_{n}<\frac{\left|f\left(b_{n}\right)-f\left(a_{n}\right)\right|}{n}<\frac{2}{n}
$$

et que

$$
f(\xi)=-\infty \text { pour } \xi=\lim _{n \rightarrow \infty} a_{n}, \text { c.q.f.d. }
$$

THÉORÈME 2. Une fonction finie $f(x)$ ayant la propriété de Darboux admettant une dérivée finie ou infinie à l'exception des points d'un ensemble au plus dénombrable et telle que $f^{\prime}(x) \geqq 0$ presque partout est continue non décroissante.

REMARQUE. Il est facile de faire voir qu'aucune de ces suppositions ne peut être omise. De même on ne peut pas remplacer $f^{\prime}(x)$ par la dérivée approximative.

Démonstration. Soit $\phi(x)$ une fonction remplissant les inégalités 


$$
\underline{f}(x) \leqq \phi(x) \leqq \bar{f}(x)
$$

que je détermine arbitrairement (si $f(x)<\bar{f}(x)$ ) en lui imposant la seule restriction que si $f(x) \leqq-1 \leqq \vec{f}(x)$, alors $\phi(x)=-1$. Evidemment partout où $f^{\prime}(x)$ existe $\phi(x)=f^{\prime}(x)$. Les ensembles $\{\phi(x) \geqq a\},\{\phi(x) \leqq a\}$ et par suite aussi $\{\phi(x)=a\}$ sont du type $G_{\delta}$. En effet, nous avons

$$
\{\phi(x) \geqq a\}=\{\bar{f}(x) \geqq a\}-\Omega_{1}, \quad\{\phi(x) \leqq a\}=\{\underline{f}(x) \leqq a\}-\Omega_{2},
$$

où $\Omega_{1}, \Omega_{2}$ sont des ensembles au plus dénombrables, donc du type $F_{\sigma}$. Il résulte du Lemme 8 que $\{\phi(x) \geqq a\}$ est $G_{\delta}$. Prenons en considération l'ensemble $A=\{\phi(x)<0\}$ et supposons que $A \neq 0$. Il contient les ensembles $B=\{\phi(x)=-1\}$ et $C=\{\phi(x)=-\infty\}$ lesquels, comme je vais le montrer, ne sont pas vides. Les ensembles $B$ et $C$ sont du type $G_{\delta}$. Tout point $x_{0} \in A$ est un point d'accumulation des ensembles $B$ et $C$. En effet, l'intervalle $\left(x_{0}-\epsilon, x_{0}\right)$ (évent. $\left(x_{0}, x_{0}+\epsilon\right)$ ) renferme les points $\xi$ tels que $f(\xi)=-\infty$ (Lemme 10), car dans le cas contraire $f(x)$ serait une fonction non décroissante dans $\left(x_{0}-\epsilon, x_{0}\right)$ et $\left(x_{0}, x_{0}+\epsilon\right)$ (ces intervalles sont les sommes d'une suite croissante d'intervalles fermés). Les limites $\lim _{x \rightarrow x_{0}^{-}} f(x), \lim _{x \rightarrow x_{0}^{+}} f(x)$ existeraient et puisque $f(x)$ a la propriété de Darboux, on aurait $f\left(x_{0}\right)=\lim _{x \rightarrow x_{0}^{-}} f(x)=\lim _{x \rightarrow x_{0}^{+}} f(x)$, c'est-à-dire que $f(x)$ serait non décroissante dans $\left(x_{0}-\epsilon, x_{0}+\epsilon\right)$ et $f\left(x_{0}\right) \geqq 0$, $\phi\left(x_{0}\right) \geqq 0, x_{0} \notin A$ contrairement à la supposition faite. $\epsilon$ étant un nombre positif arbitraire et $\phi(\xi) \leqq-1$ (d'après la définition de la fonction $\phi(x)$, car $\underline{f}(\xi)=-\infty)$, l'ensemble $A$ est dense en soi et l'ensemble $\bar{A}$ est parfait. L'ensemble $\{f(x)=-\infty\}$ est dense sur $A$; donc il est dense aussi dans $\bar{A}$. Or l'ensemble $\{f(x)=-\infty\} \bar{A}$ étant $G_{\delta}$ (Lemme 8) et étant contenu dans l'ensemble parfait $\bar{A}$, et dense sur lui a la puissance du continu sur chaque segment de $\bar{A}$. L'ensemble des points de non-dérivabilité étant tout au plus dénombrable, l'ensemble $\left\{f^{\prime}(x)=-\infty\right\}$ et à fortiori $C=\{\phi(x)=-\infty\} \subset A$ sont denses dans $\bar{A}$; en particulier tout point $x \in A$ est un point d'accumulation de l'ensemble $C$ (et cela avec la dérivée $=-\infty$ ). La fonction $f(x)$ est de I classe de Baire puisqu'elle est différentiable partout à l'exception d'un ensemble de points au plus dénombrable et comme telle possède $\leqq \boldsymbol{N}_{0}$ points de discontinuité (Lemme 8). Il s'ensuit qu'elle est de classe $\mathcal{F}$. Je vais montrer que dans chaque voisinage $\left(x_{0}-\epsilon, x_{0}+\epsilon\right)$ du point $x_{0} \in A$ se trouve un point de l'ensemble $B$. On sait déjà que le point de dérivabilité $x_{1} \in C$ appartient à ce voisinage, on a donc $f^{\prime}\left(x_{1}\right)=-\infty$. Supposons p.ex. que $x_{1}<x_{0}$ (si $x_{0}<x_{1}$ la preuve est semblable). D'après la supposition il existe un point $x_{2} \in\left(x_{1}, x_{0}\right)$ tel que $f^{\prime}\left(x_{2}\right) \geqq 0>-1$. Il s'ensuit, d'après le corollaire du Lemme 9 , qu'il y a dans $\left(x_{1}, x_{2}\right)$ un point $\xi$ tel que

$$
\underline{f}(\xi) \leqq-1 \leqq \bar{f}(\xi)
$$

donc en vertu de la définition de $\phi(x)$ on a $\phi(\xi)=-1$, c'est-à-dire que $\xi$ $\in B\left[\left(x_{0}-\epsilon, x_{0}\right)+\left(x_{0}, x_{0}+\epsilon\right)\right]$. 
Puisque $B \subset A \subset \bar{A}, B$ est pareillement dense sur $\bar{A}$. On en déduit que $B$ et $C$ qui sont des ensembles du type $G_{\delta}$ denses sur $\bar{A}$ possèdent les complémentaires de I catégorie par rapport à $\bar{A}$. Il existe donc un point $z \in B C$, où

$$
-\infty=\underline{f}(z) \leqq \bar{f}(z)<-1, \quad \underline{f}(z) \leqq-1 \leqq \bar{f}(z)
$$

ce qui est impossible. Il résulte de cette contradiction que $A=0$ donc $f(x)>-1$ pour tout $x$. De là s'ensuit en vertu du Lemme 10 que $f(x)$ est noñ décroissante; possédant la propriété de Darboux, elle doit être continue, c.q.f.d.

Corollaire. Les fonctions $f(x)$ singulières (c-à-d. continues de classe VB et telles que $f^{\prime}(x)=0$ presque partout) non constantes (en particulier les fonctions singulières élémentaires, $c-\grave{a}-d$. celles dont l'ensemble des intervalles de constance est dense partout et de mesure pleine) ont l'ensemble non-dénombrable $\left(2^{\mathrm{N}_{0}}\right)$ des points de non-dérivabilité $(f(x)<\bar{f}(x))$.

Pour les fonctions singulières élémentaires de Lebesgue, Cantor, Harnack c'est connu (Ruziewicz [24]).

ThÉOR ̀̀me 3. La dérivée d'une fonction continue et partout dérivable (event. avec la dérivée infinie) est de classe $\mathfrak{X}_{2}$.

Démonstration. Les ensembles $\left\{f^{\prime}(x)>a\right\},\left\{f^{\prime}(x)<a\right\}$ sont du type $F_{\sigma}$ pour chaque $a$. Pour $|a|=+\infty$ est evident que $\left\{f^{\prime}(x)>a\right\} \in M_{2},\left\{f^{\prime}(x)<a\right\} \in M_{2}$. Si $\left\{f^{\prime}(x)>a\right\} \neq 0,|a|<+\infty$, supposons que $f^{\prime}(x)$ ne remplit pas la condition $\mathcal{S i}_{2}^{*}$, il y aurait un point $x_{0}$ tel que $f^{\prime}\left(x_{0}\right)>a$ et dans un certain voisinage unilatéral p.ex. $\left(x_{0}-\delta, x_{0}\right)$ on aurait presque partout $f^{\prime}(x) \leqq a$. La fonction $f(x)-a x$ serait donc (d'après le Théorème 2) non croissante dans $\left(x_{0}-\delta, x_{0}\right)$ et $f^{-}\left(x_{0}\right)=f^{\prime}\left(x_{0}\right) \leqq a$ contrairement à la supposition que $f^{\prime}\left(x_{0}\right)>a$. Il s'ensuit de cette contradiction que $f^{\prime}(x) \in \mathcal{X}_{2}$ et pareillement $f^{\prime}(x) \in \cdot \mathscr{X}_{2}$ c'est-à-dire que $f^{\prime}(x) \in \mathscr{X}_{2}$ c.q.f.d.

Pour les fonctions continues dérivables partout hormis un ensemble dénombrable de points et les dérivées intermédiaires arbitraires (c'est-àdire des fonctions telles que $f(x) \leqq f_{p}^{\prime}(x) \leqq \bar{f}(x)$ ) on pourrait démontrer d'une manière pareille que dans chaque voisinage de $x_{0} \in\left\{f_{p}^{\prime}(x)>a\right\}$ se trouve une partie de l'ensemble $\left\{f_{p}^{\prime}(x)>a\right\}$ ayant la mesure extérieure positive mais comme on le fait voir facilement par des exemples (p.ex. $f(x)=|x|$ ) on ne peut pas affirmer qu'il en soit de même dans tout voisinage à droite et à gauche. Pour les fonctions discontinues dérivables partout le théorème, même ainsi affaibli, serait faux (p.ex. pour $f(x)=-x+\operatorname{sign} x$ ).

THÉOR ÈME 4. L'ensemble $\left\{a<f^{\prime}(x)<b\right\}$ pour une fonction continue ayant partout la dérivée finie ou infinie remplit la condition $M_{3}$ pour toutes les valeurs $a, b,-\infty \leqq a \leqq b \leqq+\infty$.

Démonstration. Tous les cas, à part le cas banal $a=b$. se ramènent au 
cas $a=0, b=1$. Si $a$ et $b$ sont finis il suffit de considérer la fonction $(f(x)-a x) /(b-a)$; si $a$ est fini et $b=+\infty$, je considère au lieu de $f(x)$ la fonction $f(x)-a x$ et je ramène ce cas au $a=0, b=+\infty$; si $b$ est fini, $a=-\infty$, nous avons $\left\{-\infty<f^{\prime}(x)<b\right\}=\left\{0<(-f(x)+b x)^{\prime}<+\infty\right\}$; enfin si $a=-\infty$, $b=+\infty$ on a $\left\{-\infty<f^{\prime}(x)<+\infty\right\}=\left\{-\infty<f^{\prime}(x)<1\right\}+\left\{0<f^{\prime}(x)<+\infty\right\}$ donc ce cas se ramène d'après le Lemme 1 aux cas précédents. Or le second de deux cas qui restent en fin de compte se ramène au premier en appliquant le Lemme 1 puisque $\left\{0<f^{\prime}(x)<+\infty\right\}=\sum_{k=1}^{\infty}\left\{0<f^{\prime}(x)<k\right\}$.

L'ensemble $A=\left\{0<f^{\prime}(x)<1\right\}$ est du type $F_{\sigma}$ et s'il n'est pas vide il est une somme des ensembles $\phi_{n}$ fermés non vides. Prenons en considération le point $x_{0} \in \phi_{n}, f^{\prime}\left(x_{0}\right)=\alpha \in(0,1)$. On a

$$
\begin{aligned}
f\left(x_{0}+h\right) & =f\left(x_{0}\right)+h \alpha+h \epsilon_{1}, \\
f\left(x_{0}+h+h_{1}\right) & =f\left(x_{0}\right)+h \alpha+h_{1} \alpha+\left(h+h_{1}\right) \epsilon_{2}, \\
\frac{f\left(x_{0}+h+h_{1}\right)-f\left(x_{0}+h\right)}{h_{1}} & =\alpha+\frac{h}{h_{1}}\left(\epsilon_{2}-\epsilon_{1}\right)+\epsilon_{2},
\end{aligned}
$$

où $\epsilon_{1} \rightarrow 0$ et $\epsilon_{2} \rightarrow 0$ quand $h$ et $h_{1} \rightarrow 0$. Je choisis une constante arbitraire positive $c$ et je suppose que $h / h_{1}<c, h h_{1}>0$. Dans ce cas il existe un nombre $\epsilon\left(x_{0}, c\right)$ $>0$ tel que pour $\left|h+h_{1}\right|<\epsilon\left(x_{0}, c\right)$ on a

$$
\left|\epsilon_{2}-\epsilon_{1}\right| c+\left|\epsilon_{2}\right|<\min \left(\frac{\alpha}{2}, \frac{1-\alpha}{2}\right)
$$

donc

$$
0<\frac{\alpha}{2}<\frac{f\left(x_{0}+h+h_{1}\right)-f\left(x_{0}+h\right)}{h_{1}}<\frac{\alpha+1}{2}<1
$$

pour $\left|h+h_{1}\right|<\epsilon\left(x_{0}, c\right), h / h_{1}<c, h h_{1}>0$. Je vais montrer que l'ensemble $A$ a une mesure positive dans l'intervalle $\left(x_{0}+h, x_{0}+h+h_{1}\right)$, c'est-à-dire que l'on peut admettre que $\eta_{n}=0$ puisque

$$
\frac{\left|A\left(x_{0}+h, x_{0}+h+h_{1}\right)\right|}{\left|h_{1}\right|}>0 .
$$

De cette manière toutes les exigences de la condition $M_{3}$ serons satisfaites. Supposons au contraire que $\left|\left(x_{0}+h, x_{0}+h+h_{1}\right) A\right|=0$ et désignons par $B^{\prime}, C^{\prime}$ les ensembles de tous les points d'accumulation des ensembles

$$
\begin{aligned}
& B=\left\{f^{\prime}(x) \geqq 1\right\}\left(x_{0}+h, x_{0}+h+h_{1}\right), \\
& C=\left\{f^{\prime}(x) \leqq 0\right\}\left(x_{0}+h, x_{0}+h+h_{1}\right) .
\end{aligned}
$$

Prenons en considération un point arbitraire $x_{1} \notin Q$, où

$$
Q=B^{\prime} C^{\prime}\left(x_{0}+h, x_{0}+h+h_{1}\right)
$$


tel que $x_{1} \in\left(x_{0}+h, x_{0}+h+h_{1}\right)$. Je prouverai qu'il appartient avec un certain voisinage à $B$ ou à $C$. Puisque $x_{1}$ n'est pas un point de l'ensemble $Q$, donc d'après (36), $x_{1} \notin B^{\prime} C^{\prime}$ et ne peut pas être un point d'accumulation de $B$ et de $C$. Nous pouvons admettre sans restreindre la généralité que $x_{1} \notin B^{\prime}$. Il y a donc un nombre $\epsilon>0$ si petit que $\left(x_{1}-\epsilon, x_{1}+\epsilon\right) \subset\left(x_{0}+h, x_{0}+h+h_{1}\right)$ et que l'intervalle $\left(x_{1}-\epsilon, x_{1}+\epsilon\right)$ contient au plus 1 point de l'ensemble $B$ (savoir évent. $x_{1} \in B$ puisque tout autre point peut être supprimé en diminuant $\epsilon$ ). On a

$$
\begin{aligned}
\left(x_{1}-\epsilon, x_{1}+\epsilon\right) & =\left(x_{1}-\epsilon, x_{1}+\epsilon\right)\left(x_{0}+h, x_{0}+h+h_{1}\right) \\
& =\left(x_{1}-\epsilon, x_{1}+\epsilon\right)\left(C+B+\left(x_{0}+h, x_{0}+h+h_{1}\right) A\right),
\end{aligned}
$$

car en chaque point soit $f^{\prime}(x) \leqq 0$, soit $0<f^{\prime}(x)<1$, soit $1 \leqq f^{\prime}(x)$; donc en tenant compte de $\left|\left(x_{0}+h, x_{0}+h+h_{1}\right) A\right|=0,\left|\left(x_{1}-\epsilon, x_{1}+\epsilon\right) B\right|=0$ on parvient à

$$
2 \epsilon=\left|\left(x_{1}-\epsilon, x_{1}+\epsilon\right) C\right|,
$$

ce qui veut dire que $f^{\prime}(x) \leqq 0$ presque partout dans $\left(x_{1}-\epsilon, x_{1}+\epsilon\right)$. En vertu du Théorème 2 on a donc partout dans $\left(x_{1}-\epsilon, x_{1}+\epsilon\right) f^{\prime}(x) \leqq 0$ et également $x_{1} \notin B$, c'est-à-dire

$$
\left.\left(x_{1}-\epsilon, x_{1}+\epsilon\right) \subset C \text { (évent. } \subset B \text { si } x_{1} \notin C^{\prime}\right) .
$$

Il s'ensuit de (37) que $x_{1} \in B+C$, donc $x_{1} \notin A$ et de là

$$
\left(x_{0}+h, x_{0}+h+h_{1}\right) A \subset Q \text {. }
$$

Je prouverai que $Q$ est dense en soi et renferme des ensembles $D, E$ denses aussi dans lui, où

$$
\begin{aligned}
& D=\left\{f^{\prime}(x)=1 / 2\right\}\left(x_{0}+h, x_{0}+h+h_{1}\right), \\
& E=\left\{f^{\prime}(x)=1 / 3\right\}\left(x_{0}+h, x_{0}+h+h_{1}\right) .
\end{aligned}
$$

Si $Q \neq 0$, prenons un point arbitraire $p \in Q$. Il est un point d'accumulation des ensembles $B, C$, donc dans tout voisinage de ce point se trouve le point $\xi$ tel que $f^{\prime}(\xi) \geqq 1$ et un point $\eta$ tel que $f^{\prime}(\eta) \leqq 0$. Puisque $f^{\prime}(x)$ jouit de la propriété de Darboux, il y a dans les mêmes voisinages des points $z, \tau$ tels que $f^{\prime}(z)=2^{-1} ; f^{\prime}(\tau)=3^{-1}$, c'est-à-dire des points des ensembles $D, E$. Il est évident que $D+E \subset\left(x_{0}+h, x_{0}+h+h_{1}\right) A$, donc d'après (38),

$$
D+E \subset Q \text {. }
$$

L'ensemble $Q+\left[x_{0}+h\right]+\left[x_{0}+h+h_{1}\right]$ est évidemment fermé. On peut donc trouver un ensemble parfait ( $Q$ ou sa partie) dans lequel les ensembles $D, E$ du type $G_{\delta}$ sont denses; ils ont donc un point commun,

$$
\xi \in D E, \quad f^{\prime}(\xi)=2^{-1}, \quad f^{\prime}(\xi)=3^{-1},
$$

ce qui est impossible. J'ai obtenu donc une contradiction dans le cas $Q \neq 0$. 
Mais dans le cas $Q=0 \mathrm{j}$ 'obtiens aussi une contradiction car dans ce cas d'après (38), $\left(x_{0}+h, x_{0}+h+h_{1}\right) A=0$ ce qui est en contradiction avec (35) puisque d'après la proposition de Lagrange

$$
\frac{f\left(x_{0}+h+h_{1}\right)-f\left(x_{0}+h\right)}{h_{1}}=f^{\prime}(\eta), \quad \eta \in\left(x_{0}+h, x_{0}+h+h_{1}\right) .
$$

(Evidemment, d'après (35), $\eta \in A$.) De cette contradiction résulte que $\left|\left(x_{0}+h, x_{0}+h+h_{1}\right) A\right|>0$, c.q.f.d.

Corollaire. L'ensemble $\left\{a<f^{\prime}(x)<b\right\}$ pour $f(x)$ continue est vide ou $\left|\left\{a<f^{\prime}(x)<b\right\}\right|>0$.

(C'est connu: MM. Denjoy [8], Selivanov [30], Clarkson [6].)

Il est facile de construire des exemples des fonctions continues dérivables partout à l'exception d'un ensemble de points dénombrable, pour lesquelles l'ensemble envisagé ne remplit pas même $M_{1}$ (remplit par contre $M_{0}$ pour certaines dérivées intermédiaires-pas toutes-et pour la dérivée ordinaire-ne remplit même pas $M_{0}$ puisque l'ensemble $\left\{0<f^{\prime}(x)<1\right\}$ se compose d'un seul point). Pareillement, on peut construire une fonction discontinue partout dérivable pour laquelle cet ensemble ne se compose que d'un point.

Corollaire. La dérivée partout finie appartient à la classe $\mathcal{X}_{3}$.

En effet, dans le cas considéré on a pour chaque $x$ : $-\infty<f^{\prime}(x)<+\infty$ donc $\left\{a<f^{\prime}(x)<+\infty\right\}=\left\{a<f^{\prime}(x)\right\}\left\{f^{\prime}(x)<+\infty\right\}=\left\{a<f^{\prime}(x)\right\}$ remplit $M_{3}$; pareillement $\left\{f^{\prime}(x)<a\right\}$.

THÉOR ̇̀ME 5. L'ensemble $\left\{f^{\prime}(x)>a\right\}$ pour une fonction $f(x)$ (même discontinue) ayant partout une dérivée finie ou infinie bornée supérieurement est de classe $M_{4}$. $\left(\right.$ C. $\left.-\dot{a}-d . f^{\prime}(x) \in \mathscr{X}_{4}^{*}\right)$.

Démonstration. L'ensemble $\left\{f^{\prime}(x)>a\right\}=\{f(x)>a\}$ est du type $F_{\sigma}$ (Lemme 8 , corollaire). Si $a=-\infty$ il possède la mesure pleine, remplit donc $M_{4}$ et même $M_{5}$. Il reste le cas de $a$ fini et il est loisible d'admettre $a=0$. Comme dans le Théorème $4,(35)$, on a pareillement si $f^{\prime}\left(x_{0}\right)=\alpha>0$

$$
\begin{aligned}
\frac{f\left(x_{0}+h+h_{1}\right)-f\left(x_{0}+h\right)}{h_{1}}>\frac{\alpha}{2}>0 & \text { pour } \frac{h}{h_{1}}<c, \\
& h h_{1}>0,\left|h+h_{1}\right|<\epsilon\left(x_{0}, c\right) .
\end{aligned}
$$

Puisque pour chaque $x$ on a $f^{\prime}(x)<M<+\infty$ (on peut admettre que $M \geqq 1$ ), donc

$$
\begin{aligned}
\left(f \left(x_{0}+h\right.\right. & \left.\left.+h_{1}\right)-f\left(x_{0}+h\right)\right) \operatorname{sign} h \\
& \leqq M \mid\left\{f^{\prime}(x)>0\right\}\left(x_{0}+h, x_{0}+h+h_{1} / 1 .\right.
\end{aligned}
$$


De (39), (40) résulte

$$
\frac{\left|\left\{f^{\prime}(x)>0\right\}\left(x_{0}+h, x_{0}+h+h_{1}\right)\right|}{\left|h_{1}\right|}>\frac{\alpha}{2 M}>0 .
$$

Or $\alpha \leqq M$, par conséquent $\alpha / 2 M \leqq 2^{-1}$. Puisque $\left\{f^{\prime}(x)>0\right\}=\sum_{n=1}^{\infty}\left\{f^{\prime}(x)\right.$ $>1 / n\}$ et puisque les ensembles $\left\{f^{\prime}(x)>1 / n\right\}$ sont du type $F_{\sigma}$, on déduit de (41) en y mettant $\left\{f^{\prime}(x)>1 / n\right\}=\sum_{m=1}^{\infty} F_{n m}$ et $1 / n<\alpha$ à la place de $\alpha$, pour tout $x \in F_{n m}$ que

$$
\begin{aligned}
& \frac{\left|\left\{f^{\prime}(x)>0\right\}\left(x_{0}+h, x_{0}+h+h_{1}\right)\right|}{\left|h_{1}\right|}>\frac{1}{2 n M}>0 \\
& \text { pour }\left|h+h_{1}\right|<\epsilon(x, c) \text {. }
\end{aligned}
$$

On peut poser $\eta_{n m}=1 / 2 n M \leqq 2^{-1}$. En ordonnant les suites doubles $F_{n m}$ et les $\eta_{n m}$ correspondantes en suites simples, nous obtiendrons les suites des ensembles $F_{n}$ et des nombres $\eta_{n}, 0<\eta_{n}<1$, telles que la condition $M_{4}$ sera remplie pour l'ensemble $\left\{f^{\prime}(x)>0\right\}$, c.q.f.d.

On pourrait déduire pour les fonctions dérivables partout sauf aux points d'un ensemble dénombrable une condition plus faible où l'inégalité (42) a lieu pour les $x$ pour lesquels $f^{\prime}(x)$ existe. Faute de liaison avec la condition de Darboux, ces résultats ne sont pas intéressants pour les classes des fonctions envisagées ici. Une généralisation au cas des fonctions qui ne sont pas dérivables partout (l'idèe en revient au M. A. Zygmund) ne se montra intéressante que dans le Théorème 2 .

CorollaIre. Les dérivées bornées appartiennent à la classe $\mathcal{X}_{4}$.

THÉOR Ème 6. Si $\left\{f(x)>a_{n}\right\},\left\{f(x)<b_{n}\right\}$ pour les ensembles de valeurs $a_{n}, b_{n}$ denses sur l'axe des y remplissent $M_{5}$, la fonction $f(x)$ est approximativement continue et réciproquement: si $f(x)$ est approximativement continue, alors $f(x) \in \mathcal{X}_{5}$. Les ensembles $\{a<f(x)<b\}$ sont également de classe $M_{5}$.

Corollarre. La somme de 2 fonctions de classe $\mathfrak{X}_{5}$ est aussi de classe $\mathfrak{X C}_{5}$.

Ce théorème est connu (M. Maximoff [17], [18]) et facile à vérifier. L'exemple de Lebesgue cité plus haut montre que la classe $\mathscr{X}_{0}=\mathscr{X}_{1}=\mathcal{F}$ n'est pas additive, c'est-à-dire qu'il ne résulte pas de $f(x) \in \mathcal{F}, g(x) \in \mathcal{F}$ que $f(x)$ $+g(x) \in \mathcal{F}$. Les propositions sur les propriétés additives des autres classes $\mathcal{H}_{n}$ seront données plus loin. On peut seulement observer que la classe des dérivées bornées (évent. inférieurement ou supérieurement), celle des dérivées de classe $\mathfrak{X}_{5}$ évent. ' $\mathfrak{X}_{5}$, et aussi celle des dérivées finies et infinies (à condition que pour aucun $x$ on n'obtient pas une forme indéterminée $\infty-\infty)$ sont additives. Au cas des formes indéterminées $\infty-\infty$ quand $f(x)+g(x)$ peut être non-dérivable pour certains $x$ bien que $f^{\prime}(x)$ et $g^{\prime}(x)$ existent pour tout $x$, se rattachent les exemples connus des fonctions continues dérivables partout 
qui ne sont pas absolument continues et $f(x)-g(x) \neq$ const. quoique $f^{\prime}(x)$ $=g^{\prime}(x)$ pour tout $x$, Ruziewicz [25], [26], Hahn [10].

La condition $M_{3}$ peut être énoncée plus simplemént. La definition donnée plus haut ne fut énoncée d'une manière plus compliquée que pour mettre en évidence la liaison avec $M_{4}$. En effet; les nombres $\eta_{n}$ dans $M_{3}, M_{4}$ peuvent être remplacés par des nombres arbitraires ne dépassant pas les $\eta_{n}$; en particulier on peut poser $\eta_{n}=0$ pour tout $n$ dans $M_{3}$ et ne pas introduire les ensembles $F_{n}$ (car dans ce cas chaque représentation de l'ensemble $F_{\sigma}$ comme une somme des ensembles fermés remplit les conditions désirées). La condition $M_{3}$ ainsi simplifiée est: l'ensemble $E$ est $d u$ type $F_{\sigma}$; pour chaque $x \in E$ et chaque $c>0$ il existe $\epsilon(x, c)>0$ tel que pour tous les $h, h_{1}$ remplissant les conditions $h h_{1}>0, h / h_{1}<c,\left|h+h_{1}\right|<\epsilon(x, c)$ on $a$

$$
\frac{\left|E\left(x+h, x+h+h_{1}\right)\right|}{\left|h_{1}\right|}>0 .
$$

(La forme équivalente $M_{3}^{\prime}$. avec $h h_{1} \geqq 0, h / h_{1} \leqq c$.) On voit de même que l'on obtient la condition $M_{2}$ si l'on pose dans $M_{3}^{\prime} \cdot c=0$ au lieu de $c>0$ (c'est-àdire $h=0)$. En effet, il y aura alors pour tout $x \in E$ un $\epsilon(x)>0$ tel que si $\left|h_{1}\right|$ $<\epsilon(x)$

$$
\frac{\left|\left(x, x+h_{1}\right) E\right|}{\left|h_{1}\right|}>0 .
$$

3. Les conditions suffisantes. Je vais maintenant caractériser les ensembles $\left\{f^{\prime}(x)>a\right\}$ pour les dérivées bornées ou approximativement continues et donner les théorèmes dans un certain sens réciproques aux Théorèmes 3-6. Je me base sur quelques lemmes donnés plus bas et sur le théorème $\left(^{*}\right)$ de MM. Lusin-Menchoff (démontré dans [18]).

$\left(^{*}\right)$ Pour chaque couple des ensembles, un mesurable $A$ et un fermé $B$ tels que $A \cdot \supset B$ (c'est-d-dire $A \supset B$ et $B$ se compose des points de densité de l'ensemble $A$ ) existe un ensemble fermé $C$ tel que $A \cdot \supset C \cdot \supset B$.

Lemme 11. Pour chaque ensemble $E$ de classe $M_{5}$ existe une fonction approximativement continue (et semi-continue) $f(x)$ telle que

$$
\begin{aligned}
& 0<f(x) \leqq 1 \text { pour tous les } x \in E \text {, } \\
& f(x)=0 \text { pour tous les } x \notin E \text {. }
\end{aligned}
$$

Démonstration. Si l'ensemble $E$ est vide, il suffit de poser $f(x) \equiv 0$. Dans le cas contraire, puisque $E$ est du type $F_{\sigma}$ on a

$$
E=\sum_{n=1}^{\infty} F_{n}
$$

où $F_{n}$ sont les ensembles fermés non vides. Je pose 


$$
\phi_{1}=F_{1} .
$$

$E$ se composant de ses propres points de densité on a $E \cdot \supset \phi_{1}$; d'après le théorème $(*)$ il y a donc un ensemble fermé $\psi_{2}$ tel que

$$
E \cdot \supset \psi_{2} \cdot \supset \phi_{1} \text {. }
$$

Je pose $\phi_{2}=F_{2}+\psi_{2}$, dans ce cas évidemment $E \cdot \supset \phi_{2} \cdot \supset \phi_{1}$. Je définis $\phi_{n}$ pour chaque $n$ entier positif de la façon suivante: Supposons que l'on ait déjà déterminé l'ensemble fermé $\phi_{n}$ remplissant les conditions

$$
\begin{gathered}
E \cdot \supset \phi_{n} \cdot \supset \phi_{n-1}, \\
\phi_{n} \supset F_{n} .
\end{gathered}
$$

D'après le théorème $\left(^{*}\right)$ il existe un ensemble fermé $\psi_{n+1}$ tel que $E \cdot \supset \psi_{n+1}$ - $\supset \phi_{n}$. Je pose $\phi_{n+1}=\psi_{n+1}+F_{n+1}$; dans ce cas évidemment $E \cdot \supset \phi_{n+1} \cdot \supset \phi_{n}$, $\phi_{n+1} \supset F_{n+1} ; \phi_{n+1}$ est fermé; les conditions (44), (45) sont remplies pour $n+1$ en place de $n$. De (44), (43), (45) résulte

$$
E=\sum_{n=1}^{\infty} \phi_{n}
$$

Je vais définir les ensembles fermés $\phi_{n / 2^{m}}$ pour chaque $n \geqq 2^{m}(m=0,1,2, \cdots)$. Pour $m=0$ ils sont déjà determinés $\left(=\phi_{n}\right)$ et remplissent la condition

$$
\phi_{n / 2^{m}} \subset \cdot \phi_{(n+1) / 2^{m}} \text {. }
$$

Pour les autres $m$ entiers positifs je les détermine par récurrence. Supposons que pour un certain $m$ on ait déjà déterminé les ensembles $\phi_{n / 2 m}$ pour chaque $n$ entier positif $\left(n \geqq 2^{m}\right)$, remplissant la condition (47). Je pose

$$
\phi_{2 n / 2^{m+1}}=\phi_{n / 2^{m}}
$$

et je détermine $\phi_{(2 n+1) / 2^{m+1}}$ comme l'ensemble fermé, existant d'après le théorème $(*)$ et tel que

$$
\phi_{n / 2^{m}} \subset \cdot \phi_{(2 n+1) / 2^{m+1}} \subset \cdot \phi_{(n+1) / 2^{m}},
$$

ce qui est possible à cause de la condition (47). Je détermine ensuite les ensembles fermés $\phi_{\lambda}$ pour chaque nombre réel $\lambda \geqq 1$ au moyen de la formule

$$
\phi_{\lambda}=\prod_{n / 2^{m} \geqq \lambda} \phi_{n / 2^{m}}
$$

ce qui pour $\lambda=N \cdot 2^{-M}$ concorde avec la définition antérieure-d'après (48). Les ensembles $\phi_{\lambda}$ remplissent la condition

$$
\phi_{\lambda_{1}} \subset \cdot \phi_{\lambda_{2}} \text { pour } \lambda_{1}<\lambda_{2} \text {. }
$$

Et effet, il s'ensuit immédiatement de la définition de $\phi_{\lambda}$ que $\phi_{\lambda_{1}} \subset \phi_{\lambda_{2}}$ pour $\lambda_{1} \leqq \lambda_{2}$. En choisissant $n$ et $m$ tels que $\lambda_{1}<2^{-m} n<2^{-m}(n+1)<\lambda_{2}$ on a donc 
d'après (48)

$$
\phi_{\lambda_{1}} \subset \phi_{n / 2^{m}} \subset \cdot \phi_{(n+1) / 2^{m}} \subset \phi_{\lambda_{2}}
$$

donc $\phi_{\lambda_{2}} \cdot \supset \phi_{\lambda_{1}}$.

La fonction

$$
f(x)=\left\{\begin{array}{ccc}
\frac{1}{\inf \frac{E}{\lambda}\left\{x \in \phi_{\lambda}\right\}} & \text { pour tout } & x \in E, \\
0 & \text { pour tout } & x \in E
\end{array}\right.
$$

remplit les conditions du lemme. Évidemment puisque $\lambda \geqq 1$ on a inf $E_{\lambda}\left\{x \in \phi_{\lambda}\right\} \geqq 1$, donc $f(x) \leqq 1$. La fonction est déterminée car en vertu de (46) si $x_{0} \in E$, alors $x_{0} \in \phi_{n_{0}}\left(n_{0}\right.$ dépend de $\left.x_{0}\right)$; à cause de cela l'ensemble de toutes les valeurs de $\lambda$ telles que $x_{0} \in \phi_{\lambda}$ n'est pas vide et sa borne inférieure inf $E_{\lambda}\left\{x_{0} \in \phi_{\lambda}\right\}$ existe (et est $\left.\leqq n_{0}\right)$. Donc $f\left(x_{0}\right) \geqq 1 / n_{0}>0$. Il reste à montrer que $f(x)$ est approximativement continue. Elle est continue aux points $x_{0} \notin E$. En effet, en choisissant un entier positif arbitraire $N$ on a d'après (46)

$$
x_{0} \notin \phi_{N} \text {. }
$$

$\phi_{N}$ étant fermé, il existe un nombre $\delta=\delta(N)>0$ tel que

$$
\left(x_{0}-\delta, x_{0}+\delta\right) \phi_{N}=0
$$

et à cause de (49), $\left(x_{0}-\delta, x_{0}+\delta\right) \phi_{\lambda}=0$ pour chaque $\lambda \leqq N$ (d'après (51)). Pour les $x \in\left(x_{0}-\delta, x_{0}+\delta\right)$ nous avons donc $x \notin \phi_{\lambda}, \lambda \leqq N$, inf $E_{\lambda}\left\{x \in \phi_{\lambda}\right\} \geqq N$ si $x \in E$, c'est-à-dire que

$$
f(x) \leqq \frac{1}{N} \text { pour tout } x \in\left(x_{0}-\delta, x_{0}+\delta\right)
$$

(car pour $x \notin E$ on a $f(x)=0$ ) et puisque $f\left(x_{0}\right)=0$, la fonction $f(x)$ est continue au point $x_{0}$. Nous montrons ensuite que la fonction $f(x)$ est semi-continue supérieurement dans chaque point: si $x_{0} \in E$ et $f\left(x_{0}\right)=1 / l(l>1)$, on a en choisissant arbitrairement $\epsilon>0, \epsilon<l-1$

$$
\begin{gathered}
x_{0} \notin \phi_{l-\epsilon}, \quad\left(x_{0}-\delta, x_{0}+\delta\right) \phi_{l-\epsilon}=0 \text { pour } \delta=\delta(\epsilon)>0, \\
\left(x_{0}-\delta, x_{0}+\delta\right) \phi_{\lambda}=0 \quad \text { pour chaque } \lambda \leqq l-\epsilon
\end{gathered}
$$

et si $x \in\left(x_{0}-\delta, x_{0}+\delta\right) \phi_{\lambda}$ on a $\lambda>l-\epsilon$ d'où inf $E_{\lambda}\left\{x \in \phi_{\lambda}\right\} \geqq l-\epsilon$,

$$
f(x) \leqq(l-\epsilon)^{-1} \text { pour tout } x \in\left(x_{0}-\delta, x_{0}+\delta\right) E .
$$

Mais pour tout $x \in\left(x_{0}-\delta, x_{0}+\delta\right)(R-E)$ la fonction $f(x)=0$, donc $f(x)$ $\leqq(l-\epsilon)^{-1}$ pour $\left|x-x_{0}\right|<\delta$, c'est-à-dire que $f(x)$ est semi-continue supérieurement. Pour $l=1$ puisque $\sup f(x)=1$ la semi-continuité supérieure est manifeste. 
La fonction $f(x)$ est en chaque point approximativement semi-continue inférieurement. $\bar{A}$ cause de la continuité en chaque $x_{0} \notin E$ il suffit de la montrer pour tout $x_{0} \in E$. Soit $f\left(x_{0}\right)=1 / l$. Dans ce cas il s'ensuit de la définition de $f(x)$ et de (49) que

$$
x_{0} \in \phi_{l+\epsilon / 2} \text { pour tous les } \epsilon>0 .
$$

En vertu de (49) $x_{0}$ est donc un point de densité de l'ensemble $\phi_{l_{+\epsilon}}$. Mais il résulte de (50) que la fonction $f(x)$ est $\geqq(l+\epsilon)^{-1}$ aux points $x \in \phi_{l+\epsilon}$ c'est-àdire que $x_{0}$ est un point de densité de l'ensemble $\left\{f(x) \geqq(l+\epsilon)^{-1}\right\}$; donc, d'après la définition des limites approximatives, $f(x)$ est approximativement semi-continue inférieurement au point $x_{0}$, c.q.f.d.

Lemme 12. Etant donnés 3 ensembles disjoints $E_{1}, E_{2}, H$ tels que $E_{1}+E_{2}+H$ $=R$ (ensemble de tous les nombres réels finis) et $E_{1}+H, E_{2}+H$ sont de classe $M_{5}$ existe une fonction $f(x)$ approximativement continue telle que

$$
\begin{array}{rll}
f(x)=0 & \text { pour tout } & x \in E_{1}, \\
0<f(x)<1 & \text { pour tout } & x \in H, \\
f(x)=1 & \text { pour tout } & x \in E_{2} .
\end{array}
$$

Démonstration. D'après le Lemme 11 existent 2 fonctions $h(x)$ et $g(x)$ approximativement continues et telles que

$$
\begin{array}{rll}
g(x)=0 & \text { pour tout } & x \in E_{1}, \\
h(x)=0 & \text { pour tout } & x \in E_{2}, \\
0<g(x) \leqq 1 & \text { pour tout } & x \in E_{2}+H, \\
0<h(x) \leqq 1 & \text { pour tout } & x \in E_{1}+H .
\end{array}
$$

La fonction

$$
\phi(x, y)=\frac{|x|}{|x|+|y|}
$$

est déterminée et continue partout sauf au point $x=0, y=0$ et $\phi(0, y)=0$ pour tous les $y \neq 0, \phi(x, 0)=1$ pour tous les $x \neq 0$,

$$
0<\phi(x, y)<1 \text { pour tous les } x \neq 0, y \neq 0 .
$$

Il s'ensuit que

$$
f(x)=\phi(g(x), h(x))
$$

remplit toutes les conditions du lemme. Elle est déterminée puisque les ensembles $E_{1}, E_{2}$ étant disjoints, on a pas en même temps $g(x)=0, h(x)=0$; $g(x) \neq 0$ et $h(x) \neq 0$ uniquement pour tous les $x \in H$; et $f(x)$ est évidemment approximativement continue, c.q.f.d. 
Lемме 13. Si les fonctions $f(x), g(x)$ remplissent les conditions: 0 $\leqq f(x), 0 \leqq g(x) \leqq 1, \quad\{f(x)=+\infty\} \cdot\{g(x)=0\}=0, \lim _{h \rightarrow 0} h^{-1} \int_{x}^{x+h} f(t) d t=f(x)$, $\lim _{h \rightarrow 0} h^{-1} \int_{x}^{x+h} g(t) d t=g(x)$ et si $f(x)$ est approximativement continue, alors $\lim _{h \rightarrow 0} h^{-1} \int_{x}^{x+h} f(t) g(t) d t=f(x) g(x)$. (En particulier si $g(x)$ est aussi approximativement continue.)

Démonstration. Je considère d'abord le cas où $f(x)$ a une valeur finie. Je choisis un nombre arbitraire $\epsilon>0$ (on peut admettre que $\epsilon<1$ ). Il résulte de la supposition qu'il existe un nombre $\delta_{1}=\delta_{1}(\epsilon, x)>0$ tel que

$$
\left|f(x)-\frac{1}{h} \int_{x}^{x+h} f(t) d t\right|<\frac{\epsilon}{2 f(x)+5}=\eta \text { pour tout }|h|<\delta_{1} .
$$

$f(t)$ étant approximativement continue, $x$ est un point de densité de l'ensemble $A=E_{t}\{|f(x)-f(t)|<\eta\}$. Il s'ensuit qu'il existe un nombre $\delta_{2}=\delta_{2}(\epsilon, x)>0$ tel que

$$
\frac{|(x, x+h) A|}{|h|}>1-\frac{\eta}{2} \text { pour tout }|h|<\delta_{2} .
$$

Je pose $N_{1}=(x, x+h) A, \quad N_{2}=(x, x+h)-A$, alors on a $h^{-1} \int_{x}^{x+h} \phi(t) d t$ $=|h|^{-1} \int_{N_{1}} \phi(t) d t+|h|^{-1} \int_{N_{2}} \phi(t) d t$ pour toute fonction sommable $\phi(t)$, en particulier pour $\phi(t)=f(t)$ et pour $\phi(t)=f(t) g(t)$. Nous avons

$$
\begin{aligned}
& (f(x)-\eta) \frac{\left|N_{1}\right|}{|h|} \leqq \frac{1}{|h|} \int_{N_{1}} f(t) d t \leqq(f(x)+\eta) \frac{\left|N_{1}\right|}{|h|} \\
& (f(x)-\eta)\left(1-\frac{\eta}{2}\right) \leqq \frac{1}{|h|} \int_{N_{1}} f(t) d t \leqq f(x)+\eta \\
& \left|f(x)-\frac{1}{|h|} \int_{N_{1}} f(t) d t\right|<\eta+\frac{\eta}{2} f(x) \quad \text { pour tout }|h|<\delta_{2}, \\
& \left|f(x)-\frac{1}{h} \int_{x}^{x+h} f(t) d t-f(x)+\frac{1}{|h|} \int_{N_{1}} f(t) d t\right| \quad \text { pour tout }|h|<\delta_{2}, \\
& =\frac{1}{|h|} \int_{N_{2}} f(t) d t<2 \eta+\frac{\eta}{2} f(x) \quad \text { pour tout }|h|<\min \left(\delta_{1}, \delta_{2}\right),
\end{aligned}
$$

d'après (52), (54). De là et de $0 \leqq g(t) \leqq 1$ résulte

$$
\frac{1}{|h|} \int_{N_{2}} f(t) g(t) d t<2 \eta+\frac{\eta}{2} f(x) \quad \text { pour tout }|h|<\min \left(\delta_{1}, \delta_{2}\right) .
$$

Pareillement l'on a 


$$
\begin{aligned}
&(f(x)-\eta) \frac{1}{|h|} \int_{N_{1}} g(t) d t \leqq \frac{1}{|h|} \int_{N_{1}} f(t) g(t) d t \\
& \leqq \leqq(f(x)+\eta) \frac{1}{|h|} \int_{N_{1}} g(t) d t \\
& \leqq(f(x)+\eta) \frac{1}{h} \int_{x}^{x+h} g(t) d t, \\
& \max (0, f(x)-\eta) \frac{1}{|h|}\left(\left|\int_{x}^{x+h} g(t) d t\right|-\int_{N_{2}} g(t) d t\right) \leqq \frac{1}{|h|} \int_{N_{1}} f(t) g(t) d t \leqq(f(x)+\eta) \frac{1}{h} \int_{x}^{x+h} g(t) d t, \\
& \max (0, f(x)-\eta) \frac{1}{|h|}\left(\left|\int_{x}^{x+h} g(t) d t\right|-\int_{N_{2}} 1 d t\right) \\
& \leqq \frac{1}{|h|} \int_{N_{1}} f(t) g(t) d t \leqq(f(x)+\eta) \frac{1}{h} \int_{x}^{x+h} g(t) d t,
\end{aligned}
$$

car $g(t) \leqq 1$. D'après la supposition il existe un nombre $\delta_{3}=\delta_{3}(\epsilon, x)>0$ tel que $\left.|| h\right|^{-1}\left|\int_{x}^{x+h} g(t) d t\right|-g(x)|=| h^{-1} \int_{x}^{x+h} g(t) d t-g(x) \mid<\eta$ pour tout $|h|<\delta_{3}$. De là et de (56), (53) résulte

$$
\begin{aligned}
& \max (0, f(x)-\eta)\left(g(x)-\eta-\frac{\left|N_{2}\right|}{|h|}\right) \leqq \frac{1}{|h|} \int_{N_{1}} f(t) g(t) d t \\
& \leqq(f(x)+\eta)(g(x)+\eta) \\
& \text { pour tout }|h|<\delta_{3}
\end{aligned}
$$

car $\eta g(x) \leqq \eta$. De là et de (55), puisque $\eta^{2} \leqq \eta$, résulte

$$
\begin{array}{r}
\left|\frac{1}{h} \int_{x}^{x+h} f(t) g(t) d t-f(x) g(x)\right| \leqq 4 \eta+2 \eta f(x)=\frac{\epsilon}{2 f(x)+5}(2 f(x)+4)<\epsilon \\
\text { pour tout }|h|<\min \left(\delta_{1}, \delta_{2}, \delta_{3}\right),
\end{array}
$$


c-à-d. $\lim _{h \rightarrow 0} h^{-1} \int_{x}^{x+h} f(t) g(t) d t=f(x) g(x)$, c.q.f.d.

Si $f(x)=+\infty, f(x) g(x)=+\infty$ (car $g(x)=a>0$ d'après supposition faite). Il existe un nombre $\delta_{1}(x, a)=\delta_{1}>0$ tel que $h^{-1} \int_{x}^{x+h} g(t) d t \geqq 3 a / 4$ pour tout $|h|<\delta_{1}$. En désignant $B=\left\{g(t)>2^{-1} a\right\}$ je vais évaluer $|(x, x+h) B|$ pour $|h|<\delta_{1}$. On a $(3 / 4) a|h| \leqq\left|\int_{x}^{x+h} g(t) d t\right| \leqq 1$. $|(x, x+h) B|+2^{-1} a \cdot|h|$,

$$
|(x, x+h) B| \geqq \frac{a|h|}{4} \quad \text { pour tout }|h|<\delta_{1} .
$$

Je choisis un nombre arbitraire positif $m$. Puisque $f(t)$ est approximativement continue, $x$ est un point de densité de l'ensemble $A=\left\{f(t)>16 a^{-2} m\right\}$, car $f(x)=+\infty$. Il existe donc un nombre $\delta_{2}(m, x)=\delta_{2}>0$ tel que

$$
\frac{|(x, x+h) A|}{|h|}>1-\frac{a}{8} \quad \text { pour tout }|h|<\delta_{2} \text {. }
$$

Il résulte donc de $(57)$

$$
\begin{aligned}
& \frac{|(x, x+h) A B|}{|h|}>\frac{a}{8} \quad \text { pour tout }|h|<\min \left(\delta_{1}, \delta_{2}\right), \\
& \frac{1}{h} \int_{x}^{x+h} f(t) g(t) d t \geqq \frac{1}{|h|} \int_{N} f(t) g(t) d t>\frac{a}{8} \cdot \frac{16 m}{a^{2}} \cdot \frac{a}{2}=m \\
& \text { pour tout }|h|<\min \left(\delta_{1}, \delta_{2}\right)
\end{aligned}
$$

où $N=(x, x+h) A B$, c-à-d. $\lim _{h \rightarrow 0} h^{-1} \int_{x}^{x+h} f(t) g(t) d t=+\infty$, c.q.f.d.

ThÉOR Ème 7. Pour chaque suite finie des $n$ nombres réels $-\infty \leqq a_{1}<a_{2}$ $<\cdots<a_{n} \leqq+\infty$ et une suite finie des $2 n-1$ ensembles disjoints non vides $N_{1}, N_{2}, \cdots, N_{n}, H_{1}, H_{2}, H_{3}, \cdots, H_{n-1}$ tels que $N_{1}+N_{2}+\cdots+N_{n}+H_{1}$ $+H_{2}+\cdots+H_{n-1}=R$ (ensemble de tous les nombres réels finis) et $N_{1}+H_{1}$ $+N_{2}+H_{2}+\cdots+N_{k}+H_{k}, H_{k}+N_{k+1}+H_{k+1}+\cdots+N_{n-1}+H_{n-1}+N_{n}$ remplissent la condition $M_{5}$ pour $k=1,2, \cdots, n-1$ et pour toute fonction croissante finie $F(z) \geqq 0$ déterminée pour les $z \geqq 0$-il existe une fonction absolument continue $f(x)$ ayant partout la dérivée $f^{\prime}(x)$ approximativement continue et intégrable avec $F$ (ce qui veut dire que pour tous les a et b finis $\int_{a}^{b} F\left(\left|f^{\prime}(x)\right|\right) d x$ $<+\infty)$ qui satisfait aux conditions

$$
\begin{aligned}
& f^{\prime}(x)=a_{k} \quad \text { pour tout } \quad x \in N_{k}, \\
& k=1,2, \cdots, n \text {, } \\
& a_{k}<f^{\prime}(x)<a_{k+1} \quad \text { pour tout } x \in H_{k} \text {, } \\
& k=1,2, \cdots, n-1 \text {. }
\end{aligned}
$$

Si $a_{1}=-\infty$, alors $\left|N_{1}\right|=0$; si $a_{n}=+\infty$, alors $\left|N_{n}\right|=0$.

Démonstration. Je considère d'abord le cas tous les $a_{k}$ finis. D'après le Lemme 12 il existe une fonction approximativement continue $f_{k}(x)$ telle que 


$$
\begin{array}{rll}
f_{k}(x)=0 & \text { pour tout } & x \in N_{1}+H_{1}+N_{2}+H_{2}+\cdots+N_{k-1}+H_{k-1}+N_{k}, \\
0<f_{k}(x)<1 & \text { pour tout } & x \in H_{k}, \\
f_{k}(x)=1 & \text { pour tout } & x \in N_{k+1}+H_{k+1}+N_{k+2}+\cdots+N_{n-1}+H_{n-1}+N_{n} .
\end{array}
$$

La fonction

$$
f^{\prime}(x)=a_{1}+\left(a_{2}-a_{1}\right) f_{1}(x)+\left(a_{3}-a_{2}\right) f_{2}(x)+\cdots+\left(a_{n}-a_{n-1}\right) f_{n-1}(x)
$$

remplit évidemment toutes les conditions du théorème. En effet, elle est approximativement continue et, étant bornée, est la dérivée de son intégrale indéfinie.

Dans le cas où les valeurs $a_{1}$ et $a_{n}$ sont infinies, c'est-à-dire $\left|N_{1}\right|=0$ et $\left|N_{n}\right|=0$ je examine les 3 hypothèses possibles: (a) aucun $a_{k}$ n'a une valeur finie; (b) un seul $a_{2}$ est fini; (c) il y a plus qu'un nombre $a_{k}$ fini. Dans chacun de ces cas j'emploie des fonctions $\phi_{1}(x), \phi_{n}(x)$ approximativement continues, intégrables avec la fonction $F^{*}(z)=\max (|z|, F(2 z+M))$ qui en chaque point sont les dérivées de ses intégrales indéfinies, c'est-à-dire

$$
\begin{aligned}
\phi_{1}(x) & =\lim _{h \rightarrow 0} \frac{1}{h} \int_{x}^{x+h} \phi_{1}(\xi) d \xi, \\
\phi_{n}(x) & =\lim _{h \rightarrow 0} \frac{1}{h} \int_{x}^{x+h} \phi_{n}(\xi) d \xi, \\
0<\phi_{1}(x) & <+\infty \quad \text { pour tout } \quad x \in N_{1}, \\
0<\phi_{n}(x) & <+\infty \quad \text { pour tout } \quad x \in N_{n}, \\
\phi_{1}(x) & =+\infty \quad \text { pour tout } \quad x \in N_{1}, \\
\phi_{n}(x) & =+\infty \quad \text { pour tout } \quad x \in N_{n} .
\end{aligned}
$$

Ces fonctions peuvent être construites au moyen de la méthode employée dans le Lemme 11; une difficulté additionnelle consiste dans la condition (58) qui, pour les fonctions non bornées ne résulte pas de la seule continuité approximative, ainsi que dans la condition d'intégrabilité avec $F^{*}(z)$. J'ai donné la construction dans mon travail [37, Théorème $\mathrm{V}$, p. 92], résumé [35]; sans condition d'intégrabilité avec $F$-dans [38].

Dans le cas (a) je choisis la constante $M$ égale à 0 ; dans les cas (b), (c) $M=\max \left(\left|a_{2}\right|,\left|a_{3}\right|, \cdots,\left|a_{n-1}\right|\right)$.

Dans le cas (a) la fonction

$$
f^{\prime}(x)=\phi_{n}(x)-\phi_{1}(x)=\phi_{2}(x)-\phi_{1}(x)
$$

remplit évidemment toutes les conditions du théorème. Elle est définié puisque l'expression du type $\infty-\infty$ est exclue par la condition $N_{1} N_{2}=0$. Elle est intégrable avec $F(z)$ car 


$$
\begin{aligned}
\int_{a}^{b} F\left(\left|\phi_{2}(x)-\phi_{1}(x)\right|\right) d x \\
\quad \leqq \int_{a}^{b} F\left(\left|\phi_{2}(x)\right|+\left|\phi_{1}(x)\right|\right) d x \leqq \int_{a}^{b} F\left(2 \max \left[\phi_{1}(x), \phi_{2}(x)\right]\right) d x \\
\quad=\int_{E_{1}} F\left(2 \phi_{1}(x)\right) d x+\int_{E_{2}} F\left(2 \phi_{2}(x)\right) d x \\
\quad \leqq \int_{a}^{b} F\left(2 \phi_{1}(x)\right) d x+\int_{a}^{b} F\left(2 \phi_{2}(x)\right) d x<+\infty
\end{aligned}
$$

où $E_{1}=\left\{\phi_{1}(x) \geqq \phi_{2}(x)\right\} \quad(a, b), E_{2}=\left\{\phi_{2}(x)>\phi_{1}(x)\right\}(a, b)$. Dans les cas (b), (c) je forme les fonctions $g_{1}(x)$ et $g_{n}(x)$ approximativement continues et telles que

$$
\begin{array}{rll}
g_{1}(x)=0 & \text { pour tout } & x \in N_{2}+H_{2}+N_{3}+H_{3}+\cdots+H_{n-1}+N_{n}, \\
0<g_{1}(x) \leqq 1 & \text { pour tout } & x \in N_{1}+H_{1}, \\
g_{n}(x)=0 & \text { pour tout } & x \in N_{1}+H_{1}+N_{2}+H_{2}+\cdots+N_{n-1}, \\
0<g_{n}(x) \leqq 1 & \text { pour tout } & x \in H_{n-1}+N_{n} .
\end{array}
$$

D'après le Lemme 11 telles fonctions existent. En vertu du Lemme 13 les fonctions $h_{1}^{\prime}(x)=\phi_{1}(x) g_{1}(x), h_{n}^{\prime}(x)=\phi_{n}(x) g_{n}(x)$ approximativement continues sont les dérivées de ses intégrales indéfinies et remplissent d'après (59), (60) les conditions:

$$
\begin{aligned}
& h_{1}^{\prime}(x)=0 \quad \text { pour tout } \quad x \in N_{2}+H_{2}+\cdots+N_{n-1}+H_{n-1}+N_{n}, \\
& 0<h_{1}^{\prime}(x)<+\infty \text { pour tout } x \in H_{1} \text {, } \\
& h_{1}^{\prime}(x)=+\infty \text { pour tout } x \in N_{1} \text {, } \\
& h_{n}^{\prime}(x)=0 \quad \text { pour tout } \quad x \in N_{1}+H_{1}+\cdots+N_{n-1} \text {, } \\
& 0<h_{n}^{\prime}(x)<+\infty \text { pour tout } x \in H_{n-1} \text {, } \\
& h_{n}^{\prime}(x)=+\infty \text { pour tout } x \in N_{n} .
\end{aligned}
$$

Dans le cas (b) la fonction

$$
f^{\prime}(x)=h_{n}^{\prime}(x)-h_{1}^{\prime}(x)+a_{2}=h_{3}^{\prime}(x)-h_{1}^{\prime}(x)+a_{2}
$$

et dans le cas (c) la fonction

$$
f^{\prime}(x)=h_{n}^{\prime}(x)-h_{1}^{\prime}(x)+a_{2}+\left(a_{3}-a_{2}\right) f_{2}(x)+\cdots+\left(a_{n-1}-a_{n-2}\right) f_{n-2}(x)
$$

remplit toutes les conditions du théorème. Il suffit à montrer l'intégrabilité avec $F(z)$; les autres propriétés résultent des propriétés des fonctions $f_{k}(x)$, $h_{n}^{\prime}(x), h_{1}^{\prime}(x)$ déjà démontrées. On a 


$$
\begin{aligned}
F\left(\left|f^{\prime}(x)\right|\right) & \leqq F\left(\left|h_{n}^{\prime}(x)\right|+\left|h_{1}^{\prime}(x)\right|+\max \left(\left|a_{2}\right|,\left|a_{3}\right|, \cdots,\left|a_{n-1}\right|\right)\right) \\
& \leqq F\left(2 \cdot \max \left[\left|h_{n}^{\prime}(x)\right|,\left|h_{1}^{\prime}(x)\right|\right]+M\right) \\
& \leqq F\left(2 \cdot \max \left[\phi_{1}(x), \phi_{n}(x)\right]+M\right)
\end{aligned}
$$

$\left(\operatorname{car}\left|h_{1}^{\prime}(x)\right|=\left|\phi_{1}(x) \cdot g_{1}(x)\right|,\left|g_{1}(x)\right| \leqq 1\right.$, etc.). Puisque $\phi_{1}(x), \phi_{n}(x)$ sont intégrables avec $F^{*}(z) \geqq F(2 z+M)$, il en résulte, d'une manière analogue à (61), que

$$
\int_{a}^{b} F\left(\left|f^{\prime}(x)\right|\right) d x<+\infty
$$

c.q.f.d. Il reste le cas où une seule des valeurs de $a_{k}$ est infinie. Il suffit d'envisager le cas $a_{n}=+\infty$; le raisonnement pour $a_{1}=-\infty$ est semblable (on peut du reste l'obtenir du cas précédent en changeant le signe de la fonction $f(x)$, des nombres $a_{k}$ et de leur numérotage).

Je détermine alors la fonction $f^{\prime}(x)$ un peu différemment en posant

$$
f^{\prime}(x)=h_{n}^{\prime}(x)+a_{1}+\left(a_{2}-a_{1}\right) f_{1}(x)+\cdots+\left(a_{n-1}-a_{n-2}\right) f_{n-2}(x)
$$

pour $n>2$, et $f^{\prime}(x)=h_{n}^{\prime}(x)+a_{1}=h_{2}^{\prime}(x)+a_{1}$ pour $n=2$. La fonction $f(x)$ remplit toutes les conditions du théorème. La démonstration en est semblable à celle du cas précédent. En posant $M=\max \left(\left|a_{1}\right|,\left|a_{2}\right|, \cdots,\left|a_{n-1}\right|\right)$ suffirait même de prouver l'intégrabilité avec $F(z+M)$ qui s'ensuit évidemment de l'intégrabilité avec $F(2 z+M)$.

THÉOR Ème 8. Pour chaque ensemble $E$ de classe $M_{4}$ existe une fonction continue $f(x)$ ayant partout la dérivée bornée, telle que $f^{\prime}(x)=0$ pour tout $x \notin E$, $0<f^{\prime}(x)<1$ pour tout $x \in E$.

Pour 2 ensembles disjoints $E_{1}, E_{2}$ de classe $M_{4}$ il existe une fonction dérivable partout $g(x)$ telle que $-1<g^{\prime}(x)<0$ pour tout $x \in E_{1}, 0<g^{\prime}(x)<1$ pour tout $x \in E_{2}$ et $g^{\prime}(x)=0$ pour tout $x \notin E_{1}+E_{2}$.

Pour chaque couple d'ensembles disjoints $N_{1}, N_{2}$ tels que le complémentaire de l'ensemble $N_{1}$ est de classe $M_{4}$ et $N_{2}$ est $G_{\delta}$ de mésure 0 et pour chaque fonction finie croissante $F(z) \geqq 0$ déterminée pour les $z \geqq 0$ existe une fonction $h(x)$ absolument continue ayant partout la dérivée et telle que $h^{\prime}(x)$ est intégrable avec $F, h^{\prime}(x)=0$ pour tout $x \in N_{1} h^{\prime}(x)=+\infty$ pour tout $x \in N_{2}, 0<h^{\prime}(x)<+\infty$ pour tout $x \notin N_{1}+N_{2}$.

Démonstration. On peut admettre que pour chacun des ensembles $F_{n}$ tels que $\sum_{n=1}^{\infty} F_{n}=E$ qui figurent dans l'enoncé de la condition $M_{4}$ on a $\epsilon(x, 2) \geqq \alpha_{n}>0$. S'il n'en était pas ainsi, nous procederions de la façon suivante: on a, puisque $\epsilon(x, 2)>0$ pour chaque $x \in F_{n}$,

$$
F_{n}=\{\epsilon(x, 2)>0\} F_{n}=\sum_{m=1}^{\infty}\left\{\epsilon(x, 2) \geqq \frac{1}{m}\right\} F_{n}=\sum_{m=1}^{\infty} F_{n m} .
$$


Je vais démontrer, que les ensembles $F_{n m}$ sont fermés. Il suffit de montrer que le point d'accumulation $x_{0}$ des nombres $\xi_{i} \in F_{n}$ tels que $\epsilon\left(\xi_{i}, 2\right) \geqq 1 / m$ a la propriété $\epsilon\left(x_{0}, 2\right) \geqq 1 / m$. Prenons dans ce but l'intervalle $\left[x_{0}+h, x_{0}+h+h_{1}\right]$ $\subset\left(x_{0}-1 / m, x_{0}+1 / m\right)$ tel que $h h_{1}>0, h / h_{1}<2$. Posons

$$
\xi_{i}+h_{(i)}=x_{0}+h, \quad \xi_{i}+h_{(i)}+h_{1}=x_{0}+h+h_{1} .
$$

Alors pour les $\left|\xi_{i}-x_{0}\right|=\left|h-h_{(i)}\right|$ suffisamment petites on a aussi $h_{(i)} / h_{1}<2$, $h_{(i)} h_{1}>0,\left[x_{0}+h, x_{0}+h+h_{1}\right] \subset\left(\xi_{i}-1 / m, \xi_{i}+1 / m\right)$. D'autre part, $1 / m \leqq \epsilon\left(\xi_{i}, 2\right)$ donc

$$
\frac{\left|\left[x_{0}+h, x_{0}+h+h_{1}\right] E\right|}{\left|h_{1}\right|}>\eta_{n},
$$

$\operatorname{car}\left(x_{0}+h, x_{0}+h+h_{1}\right)=\left(\xi_{i}+h_{(i)}, \xi_{i}+h_{(i)}+h_{1}\right)$. Mais dans ce cas $\epsilon\left(x_{0}, 2\right) \geqq 1 / m$, c.q.f.d. On peut donc adopter au lieu de la représentation primitive de $E=\sum_{n=1}^{\infty} F_{n}$ et des nombres $\eta_{n}$ la représentation $E=\sum_{n=1}^{\infty} \sum_{m=1}^{\infty} F_{n m}$ et les nombres $\eta_{n m}=\eta_{n}$. Alors $\alpha_{n m}=1 / m$; en ordonnant ces trois suites doubles en suites simples (toutes de la même manière) nous obtiendrons la décomposition possédant la propriété voulue. Évidemment si l'on supprime les ensembles vides (éventuellement) on peut admettre que tous les $F_{n}$ sont non vides. (Si l'ensemble $E$ est vide, nous avons le cas banal dans lequel il suffit de prendre $f(x) \equiv 0$.)

On peut admettre aussi que $F_{n}$ sont bornés, car dans le cas contraire il suffirait de poser $F_{n m}=[-m, m] F_{n}, \eta_{n m}=\eta_{n}, \alpha_{n m}=\alpha_{n}$, alors $F_{n}=\sum_{m=1}^{\infty} F_{n m}$, $E=\sum_{n=1}^{\infty} \sum_{m=1}^{\infty} F_{n m}$ et en ordonnant ces trois suites doubles en suites simples, nous obtiendrions les ensembles $F_{n}^{*}$ bornés.

Je détermine la fonction $f_{n}(x)$ admettant partout la dérivée bornée, $\left|f_{n}^{\prime}(x)\right|<R_{n}$ telle que

$$
\begin{array}{rll}
f_{n}^{\prime}(x)=1 & \text { pour tout } & x \in F_{n}, \\
f_{n}^{\prime}(x)=0 & \text { pour tout } & x \notin E, \\
+\infty>R_{n}>f_{n}^{\prime}(x) \geqq 0 & \text { pour tout } & x .
\end{array}
$$

La fonction

$$
f(x)=\sum_{n=1}^{\infty} \frac{f_{n}(x)-f_{n}(0)}{2^{n} \cdot R_{n}}
$$

remplit toutes les conditions du théorème. En effet, la série $\sum_{n=1}^{\infty} f_{n}^{\prime}(x) / 2^{n} R_{n}$ $<\sum_{n=1}^{\infty} 2^{-n}=1$ est uniformément convergente et puisque

$$
\sum_{n=1}^{\infty}\left(f_{n}(0)-f_{n}(0)\right) / 2^{n} R_{n}=0,
$$

la serie (65) est uniformément convergente dans chaque intervalle fini. En vertu des propositions connues 


$$
f^{\prime}(x)=\sum_{n=1}^{\infty} \frac{f_{n}^{\prime}(x)}{2^{n} R_{n}}
$$

donc d'après (64) $0 \leqq f^{\prime}(x)<1$. De (63), (66) résulte que $f^{\prime}(x)=0$ pour chaque $x \notin E$ et de (62), (64), (66) s'ensuit que $f^{\prime}(x) \geqq 1 / 2^{n} R_{n}>0$ pour chaque $x \in F_{n}$, donc, $n$ étant arbitraire, $f^{\prime}(x)>0$ pour chaque $x \in E$. Il ne nous reste que de construire la fonction $f_{n}(x)$.

$F_{n}$ n'étant pas vide, deux composantes de son complémentaire sont les demi-droites puisque $F_{n}$ est borné et les autres composantes sont les intervalles finis. Je choisis dans chaque composante une suite des points qui converge vers ses extrémités (vers une extrémité dans le cas de demi-droite), de la façon suivante: si la composante a 2 extrémités et la longueur $l$ son milieu soit le premier de ces points; les deux suivants soient distants de $2^{-2} l$ des extrémités, les 2 suivants-distants de $2^{-3} l$ des mêmes extrémités et ainsi de suite $\left(2^{-k} l\right)$. Sur les demi-droites je choisis comme premier point le point à distance 1 , les suivants à distance $2^{-k}$ de l'extrémité. Je désigne par $I_{k}$ les segments fermés compris entre les points voisins. Si $x$ est l'extrémité la plus proche de la composante renfermant le segment $I_{k}=\left[x+h, x+h+h_{1}\right]$ on a évidemment

$$
\frac{\operatorname{dist}\left(x, I_{k}\right)}{\left|I_{k}\right|}=\frac{|h|}{\left|h_{1}\right|}=1<2
$$

et $x \in F_{n}, h h_{1}>0$, donc si $\left|h+h_{1}\right|=\left|2 h_{1}\right|<\alpha_{n}$ on a

$$
\frac{\left|E I_{k}\right|}{\left|I_{k}\right|}>\eta_{n}
$$

(c'est-à-dire quand $\left|I_{k}\right|<\alpha_{n} / 2$ ). Or le nombre des segments $I_{k}$ tels que $\left|I_{k}\right| \geqq 2^{-1} \alpha_{n}$ est fini. Cela résulte de ce que les intérieurs de ces segments sont disjoints et se trouvent tous sur un segment fini, à savoir entre le premier des points choisis sur la gauche demi-droite et le dernier sur la droite. Ils sont donc contenus dans un nombre fini des composantes du complémentaire de l'ensemble $F_{n}$. En particulier, le nombre des segments $I_{k}$ tels que

$$
\frac{\left|E I_{k}\right|}{\left|I_{k}\right|} \leqq \eta_{n}
$$

est fini. Sur chacune des composantes de l'ensemble $R-F_{n}$ (si elle est un segment fini) je choisis parmi les $I_{k}$ tels que $\left|E I_{k}\right| /\left|I_{k}\right| \leqq \eta_{n}$ un $I_{k}$ le plus à gauche et l'autre le plus à droite et j'y adjoins tous les $I_{k}$ situés entre ceux-ci (même si $\left|E I_{k}\right| /\left|I_{k}\right|>\eta_{n}$ ) et en plus le $I_{k}$ suivant à droite (sur lequel sûrement $\left.\left|E I_{k}\right| /\left|I_{k}\right|>\eta_{n}\right)$. Je désigne par $H_{i}$ les segments fermés ainsi obtenus situés dans l'intérieur des composantes de l'ensemble $R-F_{n}$. On a donc

$$
\left|E H_{i}\right|>0 \text {. }
$$


Je détermine les $H_{i}$ sur les demi-droites de la façon suivante: sur la demidroite gauche (évent. droite) je choisis $I_{k}$ tel que $\left|E I_{k}\right| /\left|I_{k}\right| \leqq \eta_{n}$, le plus éloigné à droite (évent. gauche) auquil j'adjoins le suivant à droite (évent. gauche)-donc tel que $\left|E I_{k}\right| /\left|I_{k}\right|>\eta_{n}$-ainsi que tous les points de la demidroite situés à gauche (évent. à droite) des segments ainsi choisis. S'il n'y a pas sur la demi-droite de gauche (évent. de droite) des segments $I_{k}$ tels que $\left|E I_{k}\right| /\left|I_{k}\right| \leqq \eta_{n}, H_{i}$ se compose du premier segment $I_{k}$ de gauche (évent. de droite) et de tous les points à gauche (évent. à droite) de celui-ci. (D'ailleurs si'l n'y a pas de tels $I_{k}$ sur les composantes bornées le segment $H_{i}$ n'est pas déterminé. Ainsi $H_{i}$ n'existe sûrement que sur les demi-droites et est alors lui-même une demi-droite.)

De cette manière on a déterminé dans un nombre fini des composantes du complémentaire de l'ensemble $F_{n}$ les intervalles (évent. demi-droites) fermés $H_{i}$ (un $H_{i}$ sur chacune de ces composantes). Si le nombre des composantes est infini, une infinité d'entre elles ne contiennent pas $H_{i}$. Puisque les $I_{k}$ s'accumulent vers les extrémités des composantes, les extrémités des composantes (même renfermant $H_{i}$ ) sont, comme on le déduit de la définition des $H_{i}$, des points d'accumulation de ces segments $I_{k}$ qui n'appartiennent pas aux $H_{i}$. Evidemment tous les segments $I_{k}$ n'ayant pas des points à l'intérieur de $H_{i}$ ont la propriété $\left|E I_{k}\right| /\left|I_{k}\right|>\eta_{n}$.

Je range tous les $H_{i}$ en une suite finie $H_{1}, H_{2}, \cdots, H_{k_{n}}$ et je partage ceux des $I_{k}$ qui ne se trouvent pas dans $H_{i}$ en un nombre fini de parties de la manière suivante: si chacune des moitiés $I_{k 1}, I_{k 2}$ de l'intervalle $I_{k}$ a la propriété $\left|E I_{k r}\right| / \mid I_{k r}>\eta_{n}, r=1,2$, je le partage en deux segments égales, dans le cas contraire je le laisse non partagé. Je repète ce procédé avec chacune de ces moitiés et je continue ainsi au plus $n_{k}=1+E\left(-l g_{2}\left|I_{k}\right|\right)$ fois (exactement ce nombre de fois si c'est possible). Je désigne par $L_{j}$ les segments fermés obtenus le partage fini. ( $I_{k}$ est partagé en segments en général inégaux.)

La distance $h$ d'un segment $L_{j}$ du point $x \in F_{n}$ n'est pas plus petite que la distance de $I_{k}$ renfermant ce $L_{j}$ de l'extrémité la plus proche de la composante de l'ensemble $R-F_{n}$ (=dist $\left(I_{k}, F_{n}\right)$ ), c'est-à-dire de $\left|I_{k}\right|$ comme cela résulte de la construction des segments $I_{k},(67)$. Il s'ensuit que si $L_{j}$ $=\left[x+h, x+h+h_{1}\right]$ on a $h h_{1}>0, h / h_{1} \geqq\left|I_{k}\right| /\left|L_{j}\right|$. Supposons que $L_{j}$ soit un segment résultant d'un nombre maximum des partages $n_{k}$. Dans ce cas $\left|L_{j}\right|$ $=2^{-n_{k}}\left|I_{k}\right|<2^{-l_{2}\left|I_{k}\right|^{-1}} \cdot\left|I_{k}\right|=\left|I_{k}\right|^{2}$. Je désigne par $L_{j}^{\prime}$ ces segments. On a donc

$$
\frac{\left|L_{j}^{\prime}\right|}{\operatorname{dist}\left(x, L_{j}^{\prime}\right)}=\frac{\left|L_{j}^{\prime}\right|}{|h|}=\frac{h_{1}}{h} \leqq \frac{\left|L_{j}^{\prime}\right|}{\left|I_{k}\right|}<\frac{\left|I_{k}\right|^{2}}{\left|I_{k}\right|}=\left|I_{k}\right|
$$

$$
\text { pour } L_{j}^{\prime} \subset I_{k}, x \in F_{n},
$$

$\operatorname{car}\left|h_{1}\right|=\left|L_{j}^{\prime}\right|,|h|=\operatorname{dist}\left(x, L_{j}^{\prime}\right)$. Supposons maintenant que $L_{j}$ soit un segment provenant d'un nombre de partages plus petit que $n_{k}$. Tels segments seront désignés $L_{j}^{\prime \prime}$. Dans ce cas d'après la définition du partage une des 
moitiés $L_{j r}(r=1$ ou 2$)$ de l'intervalle $L_{j}^{\prime \prime}$ a la propriété

$$
\frac{\left|L_{j r} E\right|}{\left|L_{j r}\right|} \leqq \eta_{n}
$$

Supposons que $x$ soit un point de l'ensemble $F_{n}$ et que $L_{j}^{\prime \prime}=\left[x+h, x+h+h_{1}\right]$, $L_{j r}=\left[x+k, x+k+k_{1}\right]$, alors $h h_{1}>0, k k_{1}>0$. Supposons en plus que $\left|h+h_{1}\right|$ $<\epsilon(x, c)$. Dans ce cas aussi $\left|k+k_{1}\right|<\epsilon(x, c)$, puisque $\left|k+k_{1}\right| \leqq\left|h+h_{1}\right|$. De la condition $M_{4}$ résulte que si l'on avait $k / k_{1}<c$ on aurait, contrairement à (70), $\left|L_{j r} E\right| /\left|L_{j r}\right|>\eta_{n}$. Il en résulte que

$$
k / k_{1} \geqq c .
$$

On a soit $k=h+h_{1} / 2$ soit $k=h$ et toujours $k_{1}=2^{-1} h_{1}$; donc il s'ensuit de (71) que $h / h_{1} \geqq(c-1) / 2$. Il en résulte que

$$
\begin{aligned}
& \frac{h_{1}}{h}=\frac{\left|L_{j}^{\prime}\right|}{\operatorname{dist}\left(x, L_{j}^{\prime}\right)} \leqq \frac{2}{c-1} \\
& \text { pour } L_{j}^{\prime} \subset(x-\epsilon(x, c), x+\epsilon(x, c)), x \in F_{n}, c>1 .
\end{aligned}
$$

Il s'ensuit de la définition même des intervalles $L_{j}$ que

$$
\frac{\left|E L_{j}\right|}{\left|L_{j}\right|}>\eta_{n} \text { pour tout } j .
$$

Je désigne par $E_{0}$ l'ensemble qu'on obtient de $E$ en y supprimant tous les extrémités des segments $L_{j}$ (donc celles des $H_{i}$, les extrémités des $H_{i}$ étant aussi des extrémités des $L_{j}$ adjacents). Évidemment $\left|E-E_{0}\right|=0$. Je désigne par $E_{0}^{0}$ l'ensemble de tous les points de densité de l'ensemble $E_{0}$ contenus dans $E_{0}$. D'après les propositions connues on a $\left|E_{0}-E_{0}^{0}\right|=0$. Je désigne enfin par $E^{*}$ l'ensemble $F_{\sigma}$ contenu dans $E_{0}^{0}$ tel que $\left|E_{0}^{0}-E^{*}\right|=0$. On a donc $\left|E_{0}-E^{*}\right|=0, E_{0}^{0} \supset E^{*}$ et chaque point $x \in E_{0}^{0}$ est aussi un point de densité de l'ensemble $E^{*}$, en particulier chaque point $x \in E^{*}$ est un point de densité de l'ensemble $E^{*}$, donc $E^{*}$ est de classe $M_{5}$. De la définition de $E^{*}$ résulte

$$
E^{*} \subset E_{0} \subset E, \quad\left|E-E^{*}\right|=0
$$

et de (68), (73) on tire

$$
\left|E^{*} H_{i}\right|=s_{i}>0, \quad \frac{\left|E^{*} L_{j}\right|}{\left|L_{j}\right|}>\eta_{n} .
$$

Pour deux $H_{i}$ qui sont des demi-droites, on peut même avoir $s_{i}=+\infty$. Je choisis des ensembles fermés $\phi_{i}, \psi_{j}$ l'un dans $H_{i} E^{*}$, et l'autre dans $L_{j} E^{*}$ tels que

$$
\left|\phi_{i}\right|>r_{i}=\min \left(2^{-1} s_{i}, 1\right), \quad\left|\psi_{j}\right|>2^{-1}\left|L_{j}\right| \eta_{n}
$$


Leurs complémentaires $R-\phi_{i}, R-\psi_{j}$ étant des ensembles ouverts remplissent évidemment $M_{5}$. Il résulte de $\phi_{i} \subset E^{*}, \psi_{j} \subset E^{*}$ que $R-\phi_{i} \supset R-E^{*}, R-\psi_{j}$ $\supset R-E^{*}$, c'est-à-dire $\left(R-E^{*}\right) \phi_{i}=\left(R-E^{*}\right) \psi_{j}=0$. Puisque $E^{*}=R-\left(R-E^{*}\right)$ remplit $M_{5}$, on conclut d'après le Lemme 12 qu'il existe pour trois ensembles disjoints $\phi_{i}$ (évent. $\left.\psi_{j}\right),\left(R-\phi_{i}\right) E^{*}$ (évent. $\left.\left(R-\psi_{j}\right) E^{*}\right), R-E^{*}$, dont la somme $=R$, une fonction $\phi_{i}(x)$ (évent. $\psi_{j}(x)$ ) ayant partout une dérivée approximativement continue et telle que

$$
\begin{aligned}
\phi_{i}^{\prime}(x) & =1 \text { pour tout } x \in \phi_{i}, \\
\psi_{j}^{\prime}(x) & =1 \text { pour tout } x \in \psi_{j}, \\
0<\phi_{i}^{\prime}(x) & <1 \text { pour tout } x \in\left(R-\phi_{i}\right) E^{*}, \\
0<\psi_{j}^{\prime}(x) & <1 \text { pour tout } x \in\left(R-\psi_{j}\right) E^{*}, \\
\phi_{i}^{\prime}(x) & =\psi_{j}^{\prime}(x)=0 \text { pour tout } x \in R-E^{*} .
\end{aligned}
$$

Si $H_{i}$ a deux extrémités $a_{i}, b_{i}$ ou bien si $a_{j}, b_{j}$ désignent les extrémités de l'intervalle $L_{j}$, il résulte de (78), (77), (76), (75) que

$$
\begin{aligned}
& \phi_{i}\left(b_{i}\right)-\phi_{i}\left(a_{i}\right) \geqq 1 \cdot\left|\phi_{i}\right|>r_{i}, \\
& \psi_{j}\left(b_{j}\right)-\psi_{j}\left(a_{j}\right) \geqq 1 \cdot\left|\psi_{j}\right|>2^{-1}\left|L_{j}\right| \eta_{n}=2^{-1}\left(b_{j}-a_{j}\right) \eta_{n} .
\end{aligned}
$$

Je détermine la fonction $f_{n}(x)$ pour tout $x \notin F_{n}$ (c'est-à-dire sur tous les $H_{i}, L_{j}$ ) de la manière suivante:

Si $H_{i}$ a deux extrémités $a_{i}, b_{i}$, je pose

$$
f_{n}(x)=a_{i}+\frac{b_{i}-a_{i}}{\phi_{i}\left(b_{i}\right)-\phi_{i}\left(a_{i}\right)}\left[\phi_{i}(x)-\phi_{i}\left(a_{i}\right)\right] \text { pour tout } x \in H_{i} .
$$

En particulier on a donc $f_{n}\left(a_{i}\right)=a_{i}, f_{n}\left(b_{i}\right)=b_{i}$. Puisque $a_{i} \notin E_{0}, b_{i} \notin E_{0}$ donc $a_{i} \in R-E^{*}, b_{i} \in R-E^{*}$ d'après (74). Il s'ensuit de (78) que $f_{n}^{+}\left(a_{i}\right)=f_{n}^{-}\left(b_{i}\right)=0$.

Si $H_{i}$ a une extrémité $b_{i}\left(a_{i}=-\infty\right)$, je pose

$$
f_{n}(x)=b_{i}+\phi_{i}(x)-\phi_{i}\left(b_{i}\right) \text { pour tout } x \in H_{i},
$$

on a donc $f_{n}\left(b_{i}\right)=b_{i}, f_{n}^{-}\left(b_{i}\right)=0$.

Si $H_{i}$ a une extrémité $a_{i}\left(b_{i}=+\infty\right)$, je pose

$$
f_{n}(x)=a_{i}+\phi_{i}(x)-\phi_{i}\left(a_{i}\right) \text { pour tout } x \in H_{i},
$$

on a donc $f_{n}\left(a_{i}\right)=a_{i}, f_{n}^{+}\left(a_{i}\right)=0$.

Enfin pour chaque $x \in L_{j}=\left[a_{j}, b_{j}\right]$ je pose

$$
f_{n}(x)=a_{j}+\frac{b_{j}-a_{j}}{\psi_{j}\left(b_{j}\right)-\psi_{j}\left(a_{j}\right)}\left[\psi_{j}(x)-\psi_{j}\left(a_{j}\right)\right],
$$

donc $f_{n}\left(a_{j}\right)=a_{j}, f_{n}\left(b_{j}\right)=b_{j}, f_{n}^{+}\left(a_{j}\right)=f_{n}^{-}\left(b_{j}\right)=0$ (puisque les extrémités $a_{j}, b_{j}$ de $L_{j}$ appartiennent à $\left.R-E^{*}\right)$. Bien que la fonction $f_{n}(x)$ soit. définie de deux manières aux extrémités des segments $L_{j}$ (pour deux segments voisins fermés 
ayant l'extrémité commune) les valeurs de $f_{n}(x)$ de ces deux définitions sont égales, notamment $f_{n}(x)=x$ et en ces points existe la dérivée $f_{n}^{\prime}(x)=0$, puisque $f_{n}^{+}(x)=f_{n}^{-}(x)=0$. L'existence de $f_{n}^{\prime}(x)$ à l'intérieur des segments $L_{j}$, $H_{i}$ est claire et son estimation résulte de $(76),(77),(78),(79),(80),(81)$, (82), (83). On a notamment une des inégalités suivantes

$$
\begin{aligned}
& 0 \leqq f_{n}^{\prime}(x)=\phi_{i}^{\prime}(x) \leqq 1, \\
& 0 \leqq f_{n}^{\prime}(x)=\phi_{i}^{\prime}(x) \frac{b_{i}-a_{i}}{\phi_{i}\left(b_{i}\right)-\phi_{i}\left(a_{i}\right)}<1 \cdot \frac{b_{i}-a_{i}}{r_{i}}=t_{i}, \\
& 0 \leqq f_{n}^{\prime}(x)=\psi_{j}^{\prime}(x) \frac{b_{j}-a_{j}}{\psi_{j}\left(b_{j}\right)-\psi_{j}\left(a_{j}\right)}<1 \cdot \frac{b_{j}-a_{j}}{2^{-1}\left(b_{j}-a_{j}\right) \eta_{n}}=\frac{2}{\eta_{n}} .
\end{aligned}
$$

En désignant par $R_{n}$ le nombre $\max \left(1,2 / \eta_{n}, t_{1}, t_{2}, \cdots, t_{k_{n}}\right)+1<+\infty$ on a

$$
0 \leqq f_{n}^{\prime}(x)<R_{n} \text { pour tout } x \notin F_{n},\left(R_{n}>1\right) .
$$

Je pose pour chaque $x \in F_{n}$

$$
f_{n}(x)=x
$$

et je vais démontrer que $f_{n}^{\prime}(x)=1$ pour chaque $x \in F_{n}$. Si $x+h$ est l'extrémité du segment $L_{j}$ ou $x+h \in F_{n}$ on a $f_{n}(x+h)=x+h$, donc $\left(f_{n}(x+h)-f_{n}(x)\right) / h=1$. Il suffit donc de ne considérer que le cas où $x+h$ est situé à l'intérieur de $L_{j}$. (On peut laisser de côté le cas où $x+h \in H_{i}$ car un nombre fini d'intervalles $H_{i}$ est situé à l'intérieur du complémentaire de l'ensemble $F_{n}$ donc dist $\left(\sum_{i=1}^{k_{n}} H_{i}, x\right) \geqq \operatorname{dist}\left(\sum_{i=1}^{k_{n}} H_{i}, F_{n}\right)=\delta>0$, et pour $|h|<\delta$ on a $x+h \notin \sum_{i=1}^{k_{n}} H_{i}$ et l'on peut évidemment prendre les $h$ si petits). Posons $L_{j}=\left[a_{j}, b_{j}\right], a_{j}<x+h$ $<b_{j}$. De (84) à cause de $L_{j} \subset R-F_{n}$ résulte

$$
\begin{gathered}
f_{n}\left(a_{j}\right) \leqq f_{n}(x+h) \leqq f_{n}\left(b_{j}\right), \\
0 \leqq f_{n}(x+h)-f_{n}\left(a_{j}\right) \leqq f_{n}\left(b_{j}\right)-f_{n}\left(a_{j}\right)=b_{j}-a_{j}=\left|L_{j}\right|, \\
0<x+h-a_{j}<b_{j}-a_{j} .
\end{gathered}
$$

La différence de deux nombres non-négatifs n'est pas plus grande en valeur absolue que le plus grand d'entre eux, donc

(86) $\left|f_{n}(x+h)-(x+h)\right|=\left|f_{n}(x+h)-f_{n}\left(a_{j}\right)-(x+h)+a_{j}\right|<b_{j}-a_{i}$ $\operatorname{car} f_{n}\left(a_{j}\right)=a_{j}$. De là

$$
\begin{aligned}
\left|\frac{f_{n}(x+h)-f_{n}(x)}{h}-1\right| & =\left|\frac{f_{n}(x+h)-x}{h}-1\right| \\
& =\left|\frac{f_{n}(x+h)-x-h}{h}\right|<\frac{b_{j}-a_{j}}{|h|}=\frac{\left|L_{j}\right|}{|h|},
\end{aligned}
$$

d'après (86), (85). Nous avons $|h|=\operatorname{dist}(x, x+h)>\operatorname{dist}\left(x, L_{j}\right)$, puisque 
$x+h \in$ int $L_{j}$ donc il s'ensuit de (87)

(88) $\left|\frac{f_{n}(x+h)-f_{n}(x)}{h}-1\right|<\frac{\left|L_{j}\right|}{\operatorname{dist}\left(x, L_{j}\right)} \quad$ pour $\quad x \in F_{n}, x+h \in \operatorname{int} L_{j}$.

Mais de (69), (72) résulte

$$
\lim _{h \rightarrow 0} \frac{\left|L_{j}\right|}{\operatorname{dist}\left(x, L_{j}\right)}=0 \quad \text { si } \quad x+h \in \operatorname{int} L_{j}, x \in F_{n} .
$$

Il suffit de montrer (89) séparément pour l'ensemble de segments $L_{j}^{\prime}$ et pour l'ensemble de segments $L_{j}^{\prime \prime}$ dont la somme est l'ensemble de tous les $L_{j}$. Si $x+h \in$ int $L_{j}^{\prime}$ et $h \rightarrow 0$, le point $x+h$ s'approche de l'ensemble $F_{n}$ et la longueur des segments $I_{k}$ dans lesquels il se trouve, tend vers 0 ; (notamment $\left|I_{k}\right| \leqq|h|$, puisque $x \in F_{n}, x+h \in I_{k},|x+h-x| \geqq \operatorname{dist}\left(F_{n}, I_{k}\right)$ et dist $\left(F_{n}, I_{k}\right)=\left|I_{k}\right|$, (67)-comme cela résulte de la définition même des $I_{k}$ ). Mais justement ce segment $I_{k}$ qui renferme $x+h$, renferme aussi $L_{j}^{\prime}$, donc de (69) résulte que

$$
|h|>\frac{\left|L_{j}^{\prime}\right|}{\operatorname{dist}\left(x, L_{j}^{\prime}\right)}, \quad \lim _{h \rightarrow 0} \frac{\left|L_{j}^{\prime}\right|}{\operatorname{dist}\left(x, L_{j}^{\prime}\right)}=0 .
$$

Si $x+h \in \operatorname{int} L_{j}^{\prime \prime}$, nous supposerons que $|h|<\epsilon_{m}$ où $m$ est un nombre arbitraire entier positif $\geqq 2$ et $\epsilon_{m}=2^{-1} \epsilon(x, m)$. On a donc $\epsilon_{m}>0$. L'intervalle $I_{k}$ renfermant $x+h$ se trouve alors dans $\left(x-2 \epsilon_{m}, x+2 \epsilon_{m}\right)$. En effet de ce que $|h|=\operatorname{dist}(x, x+h), x \in F_{n}, x+h \in I_{k}$ résulte que $|h| \geqq \operatorname{dist}\left(F_{n}, I_{k}\right)=\left|I_{k}\right|$, donc $\epsilon_{m}>\left|I_{k}\right|$. Mais pour chaque point $y \in I_{k}$ nous avons

$$
\begin{aligned}
|y-x|=|y-(x+h)+x+h-x| & \leqq|y-(x+h)|+|h| \\
& \leqq\left|I_{k}\right|+|h|<2 \epsilon_{m},
\end{aligned}
$$

c'est-à-dire $I_{k} \subset\left(x-2 \epsilon_{m}, x+2 \epsilon_{m}\right)$. L'intervalle $L_{j}^{\prime \prime}$ renfermant $x+h$ se trouve aussi dans $\left(x-2 \epsilon_{m}, x+2 \epsilon_{m}\right)$, de là $L_{j}^{\prime \prime} \subset(x-\epsilon(x, m), x+\epsilon(x, m))$ et de la formule (72) s'ensuit que $\left|L_{j}^{\prime \prime}\right| \cdot$ dist $\left(x, L_{j}^{\prime \prime}\right)^{-1} \leqq 2(m-1)^{-1}$ pour $|h|<\epsilon_{m}$, c'est-à-dire que $\lim _{h \rightarrow 0}\left|L_{j}^{\prime \prime}\right| \cdot \operatorname{dist}\left(x, L_{j}^{\prime \prime}\right)^{-1}=0$; de là et de (90) résulte (89). Par suite la formule (88) donne $f_{n}^{\prime}(x)=1$ pour tout $x \in F_{n}$. De (84) résulte donc

$$
0 \leqq f_{n}^{\prime}(x)<R_{n} \text { pour tout } x .
$$

De (78), (80), (81), (82), (83), (74), et de ce que $F_{n} \subset E$ résulte $f_{n}^{\prime}(x)=0$ pour tout $x \in(R-E)\left(R-F_{n}\right)$, mais $(R-E)\left(R-F_{n}\right) \supset(R-E)(R-E)=R-E$, c'està-dire que $f_{n}^{\prime}(x)=0$ pour tout $x \notin E$. La fonction $f_{n}(x)$ remplit donc les conditions $(62)-(64)$ et la première partie du théorème est demontrée.

La deuxième partie résulte immédiatement de ce qu'il existe 2 fonctions $f(x)$ et $k(x)$ ayant partout une dérivée et telles que

$$
0<f^{\prime}(x)<1 \text { pour tout } x \in E_{1},
$$




$$
\begin{array}{rll}
0<k^{\prime}(x)<1 & \text { pour tout } & x \in E_{2}, \\
f^{\prime}(x)=0 & \text { pour tout } & x \in E_{1} \text { (donc pour tout } x \in E_{2} \text { ), } \\
k^{\prime}(x)=0 & \text { pour tout } & x \in E_{2} \text { (donc pour tout } x \in E_{1} \text { ). }
\end{array}
$$

La fonction $g(x)=k(x)-f(x)$ remplit évidemment toutes les conditions du théorème.

La troisième partie résulte du Théorème 7 et de la première partie. Il existe pour l'ensemble $N_{1}$ la fonction $f(x)$ ayant partout une dérivée et telle que $f^{\prime}(x)=0$ pour tout $x \in N_{1}, 0<f^{\prime}(x)<1$ pour tout $x \in N_{1}$. On peut supposer que $N_{2} \neq 0$ (dans le cas contraire il suffirait de poser $h(x)=f(x)$ ). Puisque $N_{2}$ est $G_{\delta}$ et $\left|N_{2}\right|=0$, on a $R-N_{2} \in M_{5}$. En vertu du Théorème 7 il existe une fonction $l(x)$ absolument continue et dérivable partout, dont la dérivée est approximativement continue et intégrable avec $F(z)$ et telle que $l^{\prime}(x)=+\infty$ pour tout $x \in N_{2}, 0<l^{\prime}(x)<+\infty$ pour tout $x \in R-N_{2}$. La fonction $h^{\prime}(x)$ $=l^{\prime}(x) f^{\prime}(x) \leqq l^{\prime}(x), h^{\prime}(x) \geqq 0$ remplit évidemment, d'après le Lemme 13 , toutes les conditions du théorème. Pareillement, si $E_{1} \in M_{4}, E_{2} \in M_{4}, E_{1} E_{2}=0, N_{1}$ $\subset E_{1}, N_{2} \subset E_{2},\left|N_{1}\right|=0,\left|N_{2}\right|=0, N_{1} \in G_{\delta}, N_{2} \in G_{\delta}$ il existe une fonction $h_{1}(x)$ absolument continue telle que $h_{1}^{\prime}(x)=-\infty$ pour tout $x \in N_{1}, h_{1}^{\prime}(x)=+\infty$ pour tout $x \in N_{2},-\infty<h_{1}^{\prime}(x)<0$ pour tout $x \in E_{1}-N_{1}, 0<h_{1}^{\prime}(x)<+\infty$ pour tout $x \in E_{2}-N_{2}, h_{1}^{\prime}(x)=0$ pour tout $x \notin E_{1}+E_{2}, \int_{a}^{b} F\left(\left|h_{1}^{\prime}(x)\right|\right) d x<+\infty$ pour tous les $a, b$ finis.

Corollaire. La condition $M_{4}$ est nécessaire et suffisante pour l'ensemble $\left\{f^{\prime}(x)>a\right\}$ évent. $\left\{f^{\prime}(x)<a\right\}$ pour les fonctions ayant partout une dérivée bornée.

En particulier la condition nécessaire et suffisante pour qu'un ensemble soit un ensemble de zéros de la dérivée bornée est que sou complémentaire soit de classe $M_{4}$. Entre autres cette condition caractérise l'ensemble de zéros de la dérivée d'une fonction à une dérivée bornée si l'ensemble de zéros de la dérivée ou des intervalles de constance est dense partout (exemples de MM. Bogomołowa [1], Brodén [2], [3], Denjoy [7], Mazurkiewicz [19], [20], [21], Köpcke [12], [13], [14], [15], Pereno [22], Pompéju [23], Schoenflies [28], [29], Zalcwasser [39], Zahorski [33]).

4. Les propriêtés additives. Le théorème sur les propriétés additives des fonctions des classes $\mathscr{X}_{n}$ est basé sur les propriétés des classes $M_{n}$.

Lemme 14. Si $E_{1} \in M_{5}, E_{2} \in M_{4}$ alors $E_{1} E_{2} \in M_{4}$. Si $E_{1} \in M_{5}, E_{2} \in M_{5}$ alors $E_{1} E_{2} \in M_{5}$.

Démonstration. $E_{1}=\sum_{m=1}^{\infty} \phi_{m}, E_{2}=\sum_{n=1}^{\infty} F_{n}$ où $\phi_{m}, F_{n}$ sont des ensembles fermés et il existe des nombres positifs $\eta_{n}, \eta_{n}<1$ ayant cette propriété que pour tout $x \in F_{n}$ et $c>0$ il existe un tel nombre $\epsilon(x, c)>0$ que les inégalités $h h_{1}>0, h / h_{1}<c,\left|h+h_{1}\right|<\epsilon(x, c)$ entraînent 


$$
\frac{\left|\left(x+h, x+h+h_{1}\right) E_{2}\right|}{\left|h_{1}\right|}>\eta_{n} .
$$

Nous avons $E_{1} E_{2}=\sum_{m=1}^{\infty} \sum_{n=1}^{\infty} F_{n} \phi_{m}$. Je désigne $F_{n} \phi_{m}=\psi_{n m}$ et je pose $\eta_{n m}$ $=2^{-1} \eta_{n}$. Je vais montrer que pour les $\eta_{n m}$ aissi choisis l'ensemble $E_{1} E_{2}$ remplit $M_{4}$. (La suite double n'est pas essentielle ici; on peut, comme on l'a fait dans les démonstrations des Lemmes 1,2 et du Théorème 8 , transformer en suites simples la suite des ensembles fermés $\psi_{n m}$ et la suite des nombres $\eta_{n m}$ qui leurs correspondent.) Il faut prouver que pour tout $x \in \psi_{n m}$ et tout $c>0$ il existe $\epsilon_{1}(x, c)>0$ ayant la propriété suivante: si $h h_{1}>0, h / h_{1}<c,\left|h+h_{1}\right|<\epsilon_{1}(x, c)$ on a aussi

$$
\frac{\left|\left(x+h, x+h+h_{1}\right) E_{1} E_{2}\right|}{\left|h_{1}\right|}>\frac{\eta_{n}}{2} .
$$

Je choisis à cet effet le point $x \in \psi_{n m} \subset F_{n}$ et le nombre $c>0$. J'affirme qu'il existe un nombre $\epsilon_{2}(x, c)>0$ tel que de $h h_{1}>0, h / h_{1}<c,\left|h+h_{1}\right|<\epsilon_{2}(x, c)$ s'ensuit que

$$
\frac{\left|\left(x+h, x+h+h_{1}\right) E_{1}\right|}{\left|h_{1}\right|}>1-\frac{\eta_{n}}{2} .
$$

(Ce qui prouve incidemment, si l'on prend $x \in \phi_{m}$ au lieu de $x \in \psi_{n m}$ et p.ex. $2^{-1}$ au lieu de $\eta_{n}$, que $M_{5} \subset M_{4}$.) En effet si un tel nombre $\epsilon_{2}(x, c)$ n'existait pas, on aurait dans chaque voisinage du point $x$ des intervalles $\left(x+h, x+h+h_{1}\right)$, $h h_{1}>0$ tels que $h / h_{1}<c$, mais

$$
\frac{\left|\left(x+h, x+h+h_{1}\right) E_{1}\right|}{\left|h_{1}\right|} \leqq 1-\frac{\eta_{n}}{2} .
$$

Il s'ensuit que la densité moyenne de l'ensemble $E_{1}$ sur $\left(x-h-h_{1}, x+h+h_{1}\right)$ satisfairait l'inégalité

$$
\begin{aligned}
\frac{\left|\left(x-h-h_{1}, x+h+h_{1}\right) E_{1}\right|}{2\left|h+h_{1}\right|} & \leqq \frac{\left|\left(x-h-h_{1}, x+h\right)\right|+\left|h_{1}\right|\left(1-2^{-1} \eta_{n}\right)}{2\left|h+h_{1}\right|} \\
& =\frac{2 h+h_{1}+h_{1}\left(1-2^{-1} \eta_{n}\right)}{2 h+2 h_{1}} \\
& =1-\frac{h_{1}}{4\left(h+h_{1}\right)} \eta_{n}<1-\frac{1}{4(c+1)} \eta_{n}
\end{aligned}
$$

et puisqu'il y aurait $h$ et $h_{1}$ arbitrairement petits, la densité inférieure de l'ensemble $E_{1}$ au point $x \in \psi_{n m} \subset \phi_{m} \subset E_{1}$ serait $\leqq 1-\eta_{n} / 4(c+1)$ ce qui serait en contradiction avec la supposition que $x$ est un point de densité de l'ensemble $E_{1} \in M_{5}$. Posons $\epsilon_{1}(x, c)=\min \left(\epsilon(x, c), \epsilon_{2}(x, c)\right)>0$, alors de $h h_{1}>0, h / h_{1}<c$, 
$\left|h+h_{1}\right|<\epsilon_{1}(x, c)$ résulte (91) et (93) puisque $\left|h+h_{1}\right|<\epsilon(x, c)$ et $\left|h+h_{1}\right|$ $<\epsilon_{2}(x, c)$. On a donc pour les ensembles $S_{1}=\left(x+h, x+h+h_{1}\right)-E_{1}, S_{2}=(x+h$, $\left.x+h+h_{1}\right)-E_{2}$ :

$$
\frac{\left|S_{2}\right|}{\left|h_{1}\right|}<1-\eta_{n}, \quad \frac{\left|S_{1}\right|}{\left|h_{1}\right|}<\frac{\eta_{n}}{2}, \quad \frac{\left|S_{1}+S_{2}\right|}{\left|h_{1}\right|}<1-\frac{\eta_{n}}{2} .
$$

Il en résulte que pour l'ensemble $\left(x+h, x+h+h_{1}\right)-\left(S_{1}+S_{2}\right)=(x+h$, $\left.x+h+h_{1}\right) E_{1} E_{2}$ a lieu (92), c.q.f.d.

La deuxième partie du lemme est évidente puisque un point de densité des ensembles $E_{1}$ et $E_{2}$ est également un point de densité de l'ensemble $E_{1} E_{2}$.

ThÉOR ̀̀me 9. Si $f(x) \in \mathcal{X}_{\dot{n}}, g(x) \in \mathcal{X}_{\dot{m}}$ et $m+n>8$, alors la somme $f(x)$ $+g(x) \in \mathcal{X}_{\dot{k}}$ où $k=\min (n, m)$. Pour tous les $m, n$ tels que $m+n \leqq 8$ existent les fonctions bornées semi-continues $f(x) \in \mathcal{X}_{n}, g(x) \in \mathcal{X}_{m}$ telles que $f(x)+g(x)$

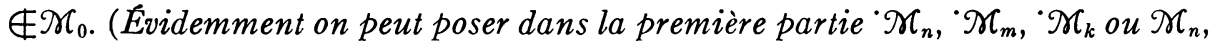
$\mathcal{X}_{m}, \mathcal{X}_{k}$ au lieu de $\mathcal{X}_{\dot{n}}, \mathcal{X}_{\dot{m}}, \mathcal{X}_{\dot{k}}$.)

Démonstration. Puisque $0 \leqq m+n \leqq 10$ l'inégalité $m+n>8$ ne peut avoir lieu que pour $m=n=5$ et pour $m=5, n=4$ (évent. $m=4, n=5$ et ce cas évidemment ne diffère pas du precedent). On tire de notre supposition que $\{f(x)>a\} \in M_{n},\{g(x)>a\} \in M_{m}$ pour chaque $a$. De la formule

$$
\begin{aligned}
\{f(x)+g(x)>a\} & =\{f(x)>a-g(x)\} \\
& =\sum_{i, v}\left\{f(x)>\frac{u+1}{v}>\frac{u}{v}>a-g(x)\right\} \\
& =\sum_{u, v}\left\{f(x)>\frac{u+1}{v}\right\}\left\{g(x)>a-\frac{u}{v}\right\}
\end{aligned}
$$

et des Lemmes 14, 1 résulte immédiatement que pour chaque $a$ on a $\{f(x)$ $+g(x)>a\} \in M_{k}$, c'est-à-dire que $f(x)+g(x) \in \mathcal{X}_{\dot{k}}$, c.q.f.d.

Dans le cas $m=n=k=5$ on peut donner une démonstration différente: la classe $\mathscr{X}_{5}$ est identique à la classe des fonctions approximativement semicontinues inférieurement qui est additive.

Je démontrerai la deuxième partie du théorème en construisant pour $m=n=4$ et $m=5, n=3$ des contre-exemples analogues aux exemples de Lebesgue cités dans l'introduction. Ce sont d'ailleurs tous les cas possibles pour $m+n=8$. Il est superflu de considérer le cas $m+n<8$ car dans ce cas à cause de l'existence de nombres entiers $u \geqq 0, v \geqq 0$ tels que $(m+u)+(n+v)$ $=8$ et $\mathcal{X}_{m+u} \subset \mathcal{X}_{m}, \mathcal{X}_{n+v} \subset \mathcal{X}_{n}$, les contre-exemples pour les classes $\mathfrak{X}_{m+u}$, $\mathcal{X}_{n+v}$, sont en même temps les contre-exemples pour les classes $\mathcal{X}_{m}$ et $\mathcal{X}_{n}$. Je partage l'intervalle $(0,1)$ au moyen de points $2^{-n}(n=1,2, \cdots)$ et je désigne par $I_{1}, I_{2}, \cdots$ les segments fermés compris entre les points voisins de partage (et év. le point $=1$ ) rangés de droite à gauche. Le segment $I_{k}$ aura alors la 
longueur $2^{-k}$ et dist $\left(0, I_{k}\right)=\left|I_{k}\right|$. Je divise le segment $I_{k}$ en $2^{-k}$ segments égaux $L_{n}$ de longueur $2^{-2 k}$. Si $L_{n} \subset I_{k}$, alors $\left|L_{n}\right| \operatorname{dist}\left(0, L_{n}\right)^{-1} \leqq 2^{-2 k}$ -dist $\left(0, I_{k}\right)^{-1}=2^{k-2 k}=2^{-k}$. On aura donc pour chaque segment $I$ contenu dans $L_{n}$ :

$$
\lim \frac{|I|}{\operatorname{dist}(0, I)}=0 \quad \text { si dist }(0, I) \rightarrow 0 .
$$

Prenons le segment $\left[h, h+h_{1}\right]$ tel que $h / h_{1}<c, h>0, h_{1}>0$ où $c$ est une constante positive arbitrairement choisie. Il existe un nombre $\epsilon(c)>0$ tel que si $h+h_{1}<\epsilon(c)$, les extrémités des segments $L_{n}$ se trouvent dans l'intérieur de $\left[h, h+h_{1}\right]$ et l'on a, en désignant par $\bar{h}$ le premier et par $\bar{h}+\bar{h}_{1}$ le dernier de ces points (extrémités),

$$
\bar{h}_{1}>2^{-1} h_{1} \text {. }
$$

Il existe d'après (94) un nombre $\epsilon(c)>0$ tel que si dist $(0, I)<\epsilon(c), I \subset L_{n}$ alors

$$
\frac{|I|}{\operatorname{dist}(0, I)}<\frac{1}{4(c+1)} .
$$

Supposons que $h+h_{1}<\epsilon(c)$ et que $\left(h, h+h_{1}\right)$ ne contienne pas des extrémités des segments $L_{n}$. Alors $I=\left[h, h+h_{1}\right] \subset L_{n}$ et dist $(0, I)=h<\epsilon(c)$. Il résulte donc de (96) que $h / h_{1}=|I|^{-1}$ dist $(0, I)>4(c+1)$, contrairement à $h / h_{1}<c$. Il existe donc des points $\bar{h}, \bar{h}+\bar{h}_{1}\left(\bar{h}_{1} \geqq 0\right)$. Puisque les segments $I^{\prime}=[h, \bar{h}]$ et $I^{\prime \prime}=\left[\bar{h}+\bar{h}_{1}, h+h_{1}\right]$ sont contenus dans les segments $L_{n}$, on peut les prendre pour $I$ dans $(94)$ et si $h+h_{1}<\epsilon(c)$, alors $h<\epsilon(c), \bar{h}+\bar{h}_{1}<\epsilon(c)$, c'est-à-dire dist $\left(0, I^{\prime \prime}\right)=\bar{h}+\bar{h}_{1}<\epsilon(c)$, dist $\left(0, I^{\prime}\right)<\epsilon(c)$. Il s'ensuit de (96) que $\left|I^{\prime}\right|$ $\cdot\left(h+h_{1}\right)^{-1} \leqq\left|I^{\prime}\right| \operatorname{dist}\left(0, I^{\prime}\right)^{-1}<[4(c+1)]^{-1}$,

$$
\begin{aligned}
& \frac{\left|I^{\prime \prime}\right|}{h+h_{1}}<\frac{1}{4(c+1)}, \frac{\left|I^{\prime}+I^{\prime \prime}\right|}{h+h_{1}}=\frac{h_{1}^{-1}\left|I^{\prime}+I^{\prime \prime}\right|}{h_{1}^{-1} h+1}<\frac{1}{2(c+1)}, \quad h_{1}^{-1} h<c, \\
& \frac{h_{1}^{-1}\left|I^{\prime}+I^{\prime \prime}\right|}{c+1}<\frac{h_{1}^{-1}\left|I^{\prime}+I^{\prime \prime}\right|}{h_{1}^{-1} h+1}, \quad h_{1}^{-1}\left|I^{\prime \prime}+I^{\prime}\right|(c+1)^{-1}<[2(c+1)]^{-1}, \\
& h_{1}^{-1}\left|I^{\prime}+I^{\prime \prime}\right|<2^{-1}, h_{1}^{-1}\left|\left[\bar{h}, \bar{h}+\bar{h}_{1}\right]\right|=\left|\left[h, h+h_{1}\right]-I^{\prime}-I^{\prime \prime}\right| h_{1}^{-1}>2^{-1}
\end{aligned}
$$

c'est-à-dire que $\bar{h}_{1}>2^{-1} h_{1}$. Je vais construire deux fonctions bornées $f_{1}(x)$, $f_{2}(x)$ de classe $\mathfrak{X}_{4}$ en choisissant pour leurs représentations géometriques sur $L_{n}$ les côtés d'un triangle isoscèle de hauteur 1 dont la base est $L_{n}$ et dont le sommet se trouve au dessus de l'axe des $x$. Je pose $f_{1}(x)=f_{2}(x)=0$ pour $x \geqq 1$ et $f_{1}(x)=f_{2}(x)=f_{1}(-x)=f_{2}(-x)$ pour $x<0$. Je pose en dernier lieu $f_{1}(0)=1$, $f_{2}(0)=0$. Alors

$$
f_{1}(x)-f_{2}(x)=\left\{\begin{array}{lll}
0 & \text { pour tout } & x \neq 0 \\
1 & \text { pour } & x=0
\end{array}\right.
$$


$f_{1}(x)-f_{2}(x) \notin \mathcal{X}_{0}$. Il reste à montrer que $f_{1}(x)$ et $f_{2}(x)$ sont de classe $\mathcal{X}_{4}$. Les ensembles $\left\{f_{1}(x)>a\right\},\left\{f_{1}(x)<a\right\}$ et les ensembles pareils pour $f_{2}(x)$ sont ouverts, ou ouverts plus un point $(=0)$. Il sont donc du type $F_{\sigma}$. En représentant l'intérieur (la partie ouverte) de l'un d'eux d'une manière quelconque comme une somme des ensembles fermés $F_{n}$, on verra facilement que l'on obtiendra toujours des ensembles $F_{n}$ remplissant les conditions énoncées dans la définition de la classe $M_{4}$. Il reste à montrer que le point 0 peut être pris comme un des ensembles $F_{n}$, c'est-à-dire qu'il existe un nombre $\eta>0$ et un nombre $\epsilon(0, c)>0$ tels que pour $h h_{1}>0, h / h_{1}<c,\left|h-h_{1}\right|<\epsilon(0, c)$ on a

$$
\frac{\left|\left\{f_{1}(x)>a\right\}\left(h, h+h_{1}\right)\right|}{\left|h_{1}\right|}>\eta .
$$

$\overline{\mathrm{A}}$ cause de la symétrie on peut se borner au cas $h>0, h_{1}>0$. On a $f_{1}(x)=f_{2}(x)$ sur $\left(h, h+h_{1}\right)$, donc je ne considère que $f_{1}(x)$. Pour $a \geqq 1$ ou $a \leqq 0$ les ensembles considérés sont vides ou de pleine mesure, ils remplissent donc $M_{5}$ et par conséquent on peut admettre que $0<a<1$. J'affirme que l'on peut prendre pour $\left\{f_{1}(x)>a\right\}$ le nombre $\eta=2^{-1}(1-a)$ et $\eta=2^{-1} a$ pour $\left\{f_{1}(x)<a\right\}$ ainsi $\epsilon(0, c)=\epsilon(c)$. En effet, pour $h+h_{1}<\epsilon(c), h / h_{1}<c$ on a, d'après (95), $\bar{h}_{1}>2^{-1} h_{1}$, le segment $\left[\bar{h}, \bar{h}+\bar{h}_{1}\right]$ se compose des segments $L_{n}$ et sur chacun d'eux la densité moyenne de l'ensemble $\{f(x)>a\}$ est $1-a$ (comme on le voit de suite en tenant compte de la similitude des triangles). Il s'ensuit que $\left|\left\{f_{1}(x)>a\right\}\left(\bar{h}, \bar{h}+\bar{h}_{1}\right)\right|=(1-a) \bar{h}_{1}>2^{-1}(1-a) h_{1},\left|\left\{f_{1}(x)>a\right\}\left(h, h+h_{1}\right)\right|$ $>2^{-1}(1-a) h_{1}$. Pour $\left\{f_{1}(x)<a\right\}$ la démonstration est analogue. De là $f_{1}(x)$ $\in \mathscr{X}_{4}, f_{2}(x) \in \mathscr{X}_{4}$.

CorollaIre. Il existe des fonctions bornées de classe $\mathfrak{X}_{4}$ que ne sont pas des dérivées.

Considérons maintenant sur chaque segment $L_{n}$ un segment $Z_{n}$ dont le centre est au milieu de $L_{n}$ (segments concentriques) et dont la longueur est $\left|Z_{n}\right|=2^{-3 k}$ si $L_{n} \subset I_{k}$, c'est-à-dire $\left|Z_{n}\right| /\left|L_{n}\right|=2^{-k}$. Je pose $f_{3}(x)=0$ pour tous les $x \in\left(R-\sum_{n=1}^{\infty} Z_{n}\right)\{x \geqq 0\}$ et je suppose que sur les segments $Z_{n}$ la fonction $f_{3}(x)$ soit représentée par les côtés égaux du triangle isoscèle dont la base est $Z_{n}$, la hauteur $=1$ et dont le sommet est situé au dessus de l'axe des $x$, en outre je suppose que $f_{3}(-x)=f_{3}(x)$, en particulier $f_{3}(0)=0$. La fonction $f_{3}(x)$ est approximativement continue. En effet elle est continue pour $x \neq 0$; le point $x=0$ est un point de densité de l'ensemble $\left\{f_{3}(x)=0\right\}$ puisque $\left|\left\{f_{3}(x) \neq 0 ; x>0\right\}\right|=\sum_{n=1}^{\infty}\left|Z_{n}\right|$ et sur le segment $(0, h)$ tel que $h \in I_{k}$ nous avons $h^{-1}\left|\sum_{n=1}^{\infty}(0, h) Z_{n}\right| \leqq 2^{-k} \cdot 2^{-k+1} \cdot 2^{k}=2^{-k+1}, \lim _{h \rightarrow 0} h^{-1}\left|\sum_{n=1}^{\infty}(0, h) Z_{n}\right|$ $=0$. Je pose $f_{4}(x)=f_{3}(x)$ pour tout $x \neq 0$ et $f_{4}(x)=1$ pour $x=0$. Une démonstration tout à fait pareille à celle exposée plus haut pour la fonction de classe $\mathcal{X}_{4}$ nous enseigne que $f_{4}(x) \in \mathscr{X}_{3}$. (Notamment puisque les segments $Z_{n}$ se trouvent sur $\left[\bar{h}, \bar{h}+\bar{h}_{1}\right]$, on a $\left|\left\{f_{4}(x)>a\right\}\left(\bar{h}, \bar{h}+\bar{h}_{1}\right)\right|>0$ pour $0<a<1$. $)$ Mais $f_{4}(x)-f_{3}(x)=0$ pour tout $x \neq 0, f_{4}(0)-f_{3}(0)=1$ c'est-à-dire $f_{4}(x)-f_{3}(x) \notin \mathcal{X}_{0}$. 
Puisque de $f(x) \in \mathcal{X}_{n}$ résulte que $-f(x) \in \mathcal{X}_{n}$, le Théorème 9 est démontré.

5. Contre-exemples. On peut poser la question si les dérivées arbitraires peuvent être présentées comme des sommes des dérivées pour lesquelles les ensembles $\{f(x)>\alpha\}$ ont été caractérisés, c'est-à-dire des dérivées approximativement continues et bornées. La réponse est négative, les contre-examples faciles (discontinues en 1 point) se trouvent dans la démonstration du Théorème 11. D'autre part il est quand même facile de montrer que certaines dérivées sont forcément approximativement continues.

THÉOR ÈME 10. La dérivée approximativement semi-continue inférieurement en $x_{0}$ et localement bornée inférieurement en $x_{0}$ est approximativement continue en $x_{0}$. (On peut admettre dans hypothèse et dans thèse les propriêtés unilatèrales.)

Démonstration. Sans restreindre la généralité, on peut supposer que $f^{\prime}(0)=0$ et faire la démonstration pour 1 point 0 seulement, car la substitution $f_{1}(x)=f\left(x+x_{0}\right)-f^{\prime}\left(x_{0}\right) \cdot x$ ramène le cas général au cas précédent si $\left[f^{\prime}\left(x_{0}\right) \mid<+\infty\right.$, d'autre part pour $f^{\prime}\left(x_{0}\right)=+\infty$ le théorème est évident. D'après la supposition, il existe un segment $(-a, a)$ tel que

$$
f^{\prime}(x)>-M \text { pour tout } x \in(-a, a), \quad 0<M<+\infty .
$$

Supposons que $f^{\prime}(x)$ ne soit pas approximativement semi-continue supérieurement au point $x=0$. On trouvera alors un nombre $t>0$ tel que 0 ne soit pas le point de densité $=0$ de l'ensemble $\left\{f^{\prime}(x)>t\right\}$, donc la densité supérieure de cet ensemble (p.ex. celle de droite) est $>\alpha>0$. (Pour la densité de gauche la preuve est analogue.) Il existe donc une telle suite $h_{n} \rightarrow 0$ de nombres positifs que

$$
\frac{\left|\left(0, h_{n}\right)\left\{f^{\prime}(x)>t\right\}\right|}{h_{n}}>\alpha .
$$

D'autre part la densité de l'ensemble $\left\{f^{\prime}(x)<-\epsilon\right\}$ au point 0 est d'après la supposition égale à 0 pour chaque $\epsilon>0$ (p.ex. pour $\epsilon=2^{-2} \alpha t$ ). Il existe donc pour tout $\eta>0$ (p.ex. pour $\eta=2^{-2} \cdot M^{-1} \alpha t$ ) un nombre $\delta>0$ tel que

$$
\frac{\left|(0, h)\left\{f^{\prime}(x)<-\epsilon\right\}\right|}{h}<\eta \text { pour tout } 0<h<\delta=\delta(\alpha, t, M, a) .
$$

(On peut admettre $\delta \leqq a$.) En particulier cette inegalité est valable avec $n \geqq N$, pour $h=h_{n}$ et $N=N(\alpha, t, M, a)$. D'après (97) et le Lemme 10, la fonction $f(x)+M x$ est non décroissante et $f^{\prime}(x)+M$ est sommable dans $(-a, a)$ et l'on a

$$
0=f^{\prime}(0) \geqq \limsup _{h \rightarrow 0+} \frac{1}{h} \int_{0}^{h} f^{\prime}(x) d x .
$$

En effet, en désignant par $g(x)+M x$ l'intégrale indéfinie de Lebesgue de la 
fonction $f^{\prime}(x)+M$, nous avons $h^{-1}(f(h)-f(0)+M h) \geqq h^{-1}(g(h)-g(0)+M h)$ $=M+h^{-1} \int_{0}^{h} f^{\prime}(x) d x$ pour tout $h>0, h<a$. Je vais évaleur inférieurement $h_{n}^{-1} \int_{0}^{h_{n}} f^{\prime}(x) d x$ pour $n \geqq N$. De (97), (98), (99) s'ensuit que

$$
\begin{aligned}
\int_{0}^{h_{n}} f^{\prime}(x) d x \geqq t \mid & \left\{f^{\prime}(x)>t\right\}\left(0, h_{n}\right)|+(-M)|\left\{f^{\prime}(x)<-\epsilon\right\}\left(0, h_{n}\right) \mid \\
& +(-\epsilon)\left|\left\{-\epsilon \leqq f^{\prime}(x) \leqq t\right\}\left(0, h_{n}\right)\right|>t \cdot \alpha h_{n}-M \eta h_{n}-\epsilon h_{n} \\
= & h_{n}\left(\alpha t-M \frac{\alpha t}{4 M}-\frac{\alpha t}{4}\right)=h_{n} \cdot \frac{\alpha t}{2},
\end{aligned}
$$

$h_{n}^{-1} \int_{0}^{h_{n}} f^{\prime}(x) d x>2^{-1} \alpha t$ et puisque $h_{n} \rightarrow 0, \lim _{\sup _{h \rightarrow 0+}} h^{-1} \int_{0}^{h} f^{\prime}(x) d x \geqq 2^{-1} \alpha t>0$, ce qui est en contradiction avec (100). Il en résulte que $f^{\prime}(x)$ est approximativement continue, c.q.f.d.

Je laisse au lecteur la construction facile d'une fonction finie sommable semicontinue et approximativement continue partout, qui ne soit pas une dérivée. Cette fonction peut même être continue sauf en un seul point. La dérivée semicontinue inférieurement et $\neq-\infty$ pour tout $x$, est localement bornée inférieurement, donc d'après le Théorème 5 et Lemmes 1,2 appartient $a \cdot \mathscr{X}_{4}$. Le théorème précédent permet d'obtenir une conclusion plus forte.

Corollaire. La dérivée $f^{\prime}(x) \neq-\infty$ pour tout $x$ et semi-continue inférieurement est approximativement continue. (Localement: si $f^{\prime}(x)$ est semicontinue en $x_{0}, f^{\prime}\left(x_{0}\right) \neq-\infty$, alors $f^{\prime}(x)$ est appr. continue en $x_{0}$.)

Pour dérivées bornées connu, Kempisty [11].

Il est facile de prouver par des exemples, même pour la dérivée finie, que le Théorème 10 devient faux si l'on y supprime la condition d'existence d'une borne locale ou celle de semi-continuité approximative. Par contre si l'on supprime dans la corollaire la supposition $f^{\prime}(x) \neq-\infty$, l'ensemble $\left\{f^{\prime}(x)>-\infty\right\}$ étant ouvert pour les $f^{\prime}(x)$ semi-continues inférieurement, l'ensemble $\left\{f^{\prime}(x)=-\infty\right\}$ est donc fermé, non dense et le corollaire reste en vigueur dans son complémentaire. Il est facile d'ailleurs de construire une fonction continue pour laquelle l'ensemble $\left\{f^{\prime}(x)=-\infty\right\}$ se compose d'un seul point $0, f^{\prime}(x)$ existe partout et est continue pour $x \neq 0$ et le corollaire est faux pour $x=0$. Il s'ensuit que dans le corollaire ici énoncé la supposition $f^{\prime}(x) \neq-\infty$ ne peut pas être supprimée.

Corollaire. Aucune des fonctions $f_{1}(x), f_{2}(x), f_{4}(x)$ (thêorème 9) n'est une dérivée.

En effet si l'une d'elles était une dérivée elle (étant semi-continue et bornée) serait de classe $\mathscr{X}_{5}$ et d'après le Théorème 9 on aurait $f_{1}(x)+f_{2}(x)$ $\in \mathscr{X}_{4} \subset \mathscr{X}_{0}, f_{3}(x)+f_{4}(x) \in \mathscr{X}_{5} \subset \mathscr{X}_{0}$. Pour une fonction du type $f_{1}(x)$ on peut obtenir facilement même la non-dérivabilité de son intégrale indéfinie pour $x=0$ (en changeant quelques triangles en trapèzes). 
THÉOR ÈME 11. Il existe une telle fonction $f(x)$ ayant partout une dérivée finie qu'il est impossible de trouver deux fonctions $a(x)$ approximativement continue et $b(x)$ sommable satisfaisant la relation $f^{\prime}(x)=a(x)+b(x)$.

Il existe une telle fonction absolument continue $F(x)$ ayant partout une dérivée finie qu'il est impossible de trouver 3 fonctions $f_{1}(x), f_{2}(x), f_{3}(x)$ ayant partont des dérivées finies et satisfaisant aux conditions: $f_{1}^{\prime}(x)$ est approximativement continue, $f_{2}^{\prime}(x) \leqq a<+\infty, f_{3}^{\prime}(x) \geqq b>-\infty$ pour tout $x$ et $F^{\prime}(x)=f_{1}^{\prime}(x)+f_{2}^{\prime}(x)$ $+f_{3}^{\prime}(x)$.

Toute fonction sommable $\phi(x)$ est la somme $f_{1}^{\prime}(x)+f_{2}^{\prime}(x)$ ò̀ $f_{1}(x), f_{2}(x)$ sont absolument continues, dérivables partout et $0 \leqq f_{1}^{\prime}(x) \leqq+\infty,-\infty \leqq f_{2}^{\prime}(x) \leqq 0$ pour tout $x$.

Démonstration. Je détermine $f^{\prime}(x)$ de la manière suivante: je partage le segment $I_{k}=\left[2^{-k}, 2^{-k+1}\right]$ en $2^{2 k}$ segments fermés égaux $L_{n}$ et chaque $L_{n}$ en 6 segments égaux; je pose sur le deuxième segment de gauche $f^{\prime}(x)=-2^{k+1}$, sur le cinquième de gauche $f^{\prime}(x)=2^{k+1}$, sur les extrémités et le milieu de $L_{n}$, $f^{\prime}(x)=0$; sur les quatre restantes parties de $L_{n}$ la fonction $f^{\prime}(x)$ est égale aux telles fonctions linéaires que $f^{\prime}(x)$ soit continue dans $L_{n}$. En outre je pose $f^{\prime}(x)=0$ pour $x \geqq 1, f^{\prime}(-x)=-f^{\prime}(x), f^{\prime}(0)=0$. Je laisse au lecteur la vérification très facile que $f^{\prime}(x)$ est une dérivée. Supposons que $f^{\prime}(x)=a(x)+b(x)$ où $a(x)$ est approximativement continue. Je montrerai que $b(x)$ n'est pas sommable. Il suffit de considérer lecasde $+\infty>a(0) \geqq-\infty$. La démonstration dans le cas $a(0)=+\infty$ est pareille. Je désigne par $A$ le nombre $a(0)+1$ pour $a(0)$ $>-\infty$, et 0 pour $a(0)=-\infty$. Alors la densité de l'ensemble $\{a(x)>A\}$ est 0 au point 0 et il s'ensuit que sa densité moyenne sur les segments (semiouverts) $I_{k}+I_{k+1}+\cdots, k>k_{0}$, est $<24^{-1}$ et sa mesure $<2\left|I_{k}\right| \cdot 24^{-1}$ puisque $\left|I_{k}+I_{k+1}+\cdots\right|=2\left|I_{k}\right|$. De là $\left|I_{k}\{a(x)>A\}\right|<12^{-1}\left|I_{k}\right|$. De la définition de la fonction $f^{\prime}(x)$ résulte que $\left|\left\{f^{\prime}(x)=2^{k+1}\right\} I_{k}\right|=6^{-1}\left|I_{k}\right|$, donc

$$
\left|\left\{f^{\prime}(x)=2^{k+1}\right\} I_{k}-\{a(x)>A\} I_{k}\right|>\left|I_{k}\right|\left(6^{-1}-12^{-1}\right) \text { pour } k>k_{0} .
$$

Supposons que $b(x)=f^{\prime}(x)-a(x)$ soit sommable. En désignant $B_{k}$ $=\left\{f^{\prime}(x)=2^{k+1}\right\} I_{k}-\{a(x)>A\} I_{k}$ on a $b(x) \geqq 2^{k+1}-A$ pour tout $x \in B_{k}$, $\int_{B_{k}} b(x) d x \geqq\left|B_{k}\right| \cdot\left(2^{k+1}-A\right)>12^{-1}\left(2-A \cdot 2^{-k}\right)$ pour $k>k_{0}$ d'après (101), puisque $\left|\bar{I}_{k}\right|=2^{-k}$ on aura, en posant $B=B_{k_{0}+1}+B_{k_{0}+2}+\cdots$

$$
\int_{B} b(x) d x>12^{-1}\left(2+2+2+\cdots-A \cdot 2^{-k_{0}}\right)=+\infty
$$

et $b(x)$ n'est pas sommable. Nous sommes arrivés à une contradiction de laquelle résulte que $b(x)$ n'est pas sommable, c.q.f.d.

Corollaire. $f^{\prime}(x) \neq a(x)+f_{1}^{\prime}(x)+f_{2}^{\prime}(x)$, àे $a(x)$ est approximativement continue, $-\infty \leqq f_{1}^{\prime}(x) \leqq a<+\infty,-\infty<b \leqq f_{2}^{\prime}(x) \leqq+\infty$. 
Je détermine $F^{\prime}(x)$ d'une manière tout à fait analogue à $f^{\prime}(x)$ mais en posant sur les segments ci-devant considerés $F^{\prime}(x)=-2 k$ et $F^{\prime}(x)=2 k$ au lieu de $-2^{k+1}$ et $2^{k+1}$ respectivement. La vérification de ce que $F^{\prime}(x)$ est une dérivée ne présente pas de difficulté, $\int_{I_{k}}\left|F^{\prime}(x)\right| d x \leqq 2 k \cdot\left|I_{k}\right|=2^{-k+1} \cdot k$ et à cause de la convergence de la série $\sum_{k=1}^{\infty} 2^{-k+1} \cdot k$ la fonction $F^{\prime}(x)$ est sommable et $F(x)$ absolument continue. Supposons que $F^{\prime}(x)=a(x)+f_{1}^{\prime}(x)$ $+f_{2}^{\prime}(x),-\infty<f_{1}^{\prime}(x) \leqq a<+\infty,-\infty<b \leqq f_{2}^{\prime}(x)<+\infty,|a(x)|<+\infty$ pour tout $x$, alors $(a(x)+a+b)+\left(f_{1}^{\prime}(x)-a\right)+\left(f_{2}^{\prime}(x)-b\right)=F^{\prime}(x)$ et l'on peut par conséquent supposer que $a=b=0$. Alors $0 \leqq f_{2}^{\prime}(x)=F^{\prime}(x)-a(x)-f_{1}^{\prime}(x)<$ $+\infty$, donc

$$
f_{2}^{\prime}(0)=\lim _{h \rightarrow 0} h^{-1} \int_{0}^{h} f_{2}^{\prime}(x) d x=\lim _{h \rightarrow 0} \frac{1}{h} \int_{0}^{h}\left(F^{\prime}(x)-a(x)-f_{1}^{\prime}(x)\right) d x
$$

puisque $f_{2}(x)$, étant absolument continue, est égale à $c+\int_{0}^{x} f_{2}^{\prime}(t) d t$.

En désignant $A=a(0)+1$, nous avons cependant pour $k>k_{0}$ sur le segment $I_{k}$ (comme dans le cas de (101))

$$
\begin{aligned}
& \left|B_{k}\right|=\left|\left\{F^{\prime}(x)=2 k\right\} I_{k}-\{a(x)>A\} I_{k}\right|>12^{-1} \cdot\left|I_{k}\right| \quad \begin{array}{rr}
\text { pour } & k>k_{0}, \\
F^{\prime}(x)-a(x)-f_{1}^{\prime}(x) \geqq 2 k-A \quad\left(f_{1}^{\prime}(x) \leqq 0\right) & \text { pour tout } \quad x \in B_{k}, \\
\int_{B_{k}} f_{2}^{\prime}(x) d x=\int_{B_{k}}\left(F^{\prime}(x)-a(x)-f_{1}^{\prime}(x)\right) d x>(2 k-A) \cdot 12^{-1} & \\
\cdot\left|I_{k}\right|=12^{-1}\left(k \cdot 2^{-k+1}-A \cdot 2^{-k}\right) & \text { pour } \quad k>k_{0},
\end{array}
\end{aligned}
$$

$\int_{I_{k}} f_{2}^{\prime}(x) d x \geqq \int_{B_{k}} f_{2}^{\prime}(x) d x$, car $F^{\prime}(x)-a(x)-f_{1}^{\prime}(x) \geqq 0$ pour tout $x$, donc, $\int_{0}^{2^{-k+1}} f_{2}^{\prime} \cdot(x) d x=\int_{I_{k}} f_{2}^{\prime}(x) d x+\int_{I_{k+1}} f_{2}^{\prime}(x) d x+\cdots>12^{-1}\left(\sum_{n=k}^{\infty} n \cdot 2^{-n+1}-A \cdot 2^{-k+1}\right)$ pour $k>k_{0}, 2^{k-1} \int_{0}^{2^{-k+1}}\left(F^{\prime}(x)-a(x)-f_{1}^{\prime}(x)\right) d x>12^{-1}\left(k+2^{-1}(k+1)+2^{-2}(k+2)\right.$ $+\cdots-A)>12^{-1}(k-A)$ pour $k>k_{0}$, de là pour $k \rightarrow \infty \lim _{\sup _{h \rightarrow 0}} h^{-1} \int_{0}^{h}\left(F^{\prime}(x)\right.$ $\left.-a(x)-f_{1}^{\prime}(x)\right) d x=+\infty$, ce qui contredit (102), car $f_{2}^{\prime}(0)$ est, d'après la supposition, finie. On conclut qu'une telle décomposition de $F^{\prime}(x)$ en trois fonctions est impossible, c.q.f.d.

Si $\phi(x)$ est sommable, les fonctions $\phi^{*}(x)=\max (\phi(x), 0), \phi_{*}(x)$ $=\min (\phi(x), 0)$ le sont également et $\phi(x)=\phi^{*}(x)+\phi_{*}(x)$. Je pose $\phi_{1}(x)$ $=\int_{0}^{x} \phi^{*}(t) d t, \phi_{2}(x)=\int_{0}^{x} \phi_{*}(t) d t$, alors $\phi_{1}(x) \geqq 0, \Phi_{2}(x) \leqq 0$ pour tout $x, \phi_{1}^{\prime}(x)$ $=\phi^{*}(x), \phi_{2}^{\prime}(x)=\phi_{*}(x)$ presque partout, c'est-à-dire pour tout $x \notin Q$, où $|Q|=0$. Je désigne par $T$ l'ensemble du type $G_{\delta}$ tel que $T \supset Q,|T|=0$, et je désigne par $\psi(x)$ une telle fonction absolument continue ayant partout une dérivée que $\left\{\psi^{\prime}(x)=+\infty\right\}=T,\left\{0<\psi^{\prime}(x)<+\infty\right\}=R-T$. Une telle fonction existe d'après le Théorème 7. Les fonctions $f_{1}(x)=\phi_{1}(x)+\psi(x), f_{2}(x)=\phi_{2}(x)$ $-\psi(x)$ remplissent toutes les conditions de notre proposition; mais la somme $f_{1}^{\prime}(x)+f_{2}^{\prime}(x)$ n'est pas déterminée sur l'ensemble $T$.

6. Les problèmes analogues. Comme il est facile de vérifier, les condi- 
tions $\mathrm{M}_{3}$ et $\mathrm{M}_{4}$ pour les ensembles $E$ du type $F_{\sigma}$ peuvent être formulées comme il suit:

$\mathrm{M}_{3}$ : l'ensemble $E$ est vide ou pour tout $x \in E$ il résulte de $I \rightarrow x,|E I|=0$ que $\lim |I| \cdot \operatorname{dist}(x, I)^{-1}=0$;

$\mathrm{M}_{4}$ : l'ensemble $E$ est vide ou il existe une telle suite des ensembles fermés $F_{n}$ et des nombres $\eta_{n}>0$ que $E=\sum_{n=1}^{\infty} F_{n}$ et pour tout $x \in F_{n}$, il résulte de $I \rightarrow x$, $|I|^{-1} \cdot|E I| \leqq \eta_{n} q u e \lim |I| \cdot \operatorname{dist}(x, I)^{-1}=0$.

La densité inférieure de l'ensemble $E$ aux points $x \in F_{n}$ est $\geqq \eta_{n}$. De là s'ensuit que la densité supérieure (et même inférieure) de l'ensemble $E$ est $>0$ en tout point $x \in E$. Pour $E=\left\{f^{\prime}(x)>a\right\}, f^{\prime}(x)$ bornée, cela résulte aussi d'un travail de Kempisty [11].

La condition (W) énoncée par moi dans $[34$, p. 500, 510] est équivalente à $\mathrm{M}_{4}$.

Outre les deux problèmes posés dans le texte, nous posons les suivants.

III. Généraliser le théorème 7 au cas des suites (ou ensembles) infinies des nombres $a_{k}$.

IV. Généraliser le théorème 8 pour le cas d'un nombre des ensembles $E_{k}$ plus grand que 2 (comme dans Théorème 7).

V. Existe-t-il pour chaque ensemble $E \in M_{3}$ une fonction $f(x)$ (évent. absolument continue) ayant partout la dérivée finie et telle que $E=\left\{f^{\prime}(x)>0\right\}$.

VI. Existe-t-il pour chaque ensemble $E \in M_{2}$ une fonction continue (éventuellement absolument continue) $f(x)$ ayant partout une dérivée et telle que $E=\left\{f^{\prime}(x)>0\right\}$.

VII. Existe-t-il pour chaque ensemble dénombrable $E$ du type $G_{\delta}$ une telle fonction discontinue $f(x)$ ayant partout une dérivée que $f^{\prime}(x)=+\infty$ pour tout $x \in E, f^{\prime}(x)=0$ pour tout $x \notin E$.

VIII. Une dérivée $f^{\prime}(x)$ (évent. finie) peut-elle être représentée comme une somme des quatre fonctions $\phi_{1}(x) \in \mathcal{X}_{5}, \phi_{2}(x) \in \cdot \mathcal{X}_{5}, \phi_{3}(x) \in \mathcal{X}_{4}, \phi_{4}(x) \in \cdot \mathcal{X}_{4}$ ? (En particulier de telle manière que $\phi_{3}(x)=f_{1}^{\prime}(x)<M<+\infty, \phi_{4}(x)=f_{2}^{\prime}(x)$ $>-M>-\infty)$. Est-il possible que $\phi_{1}(x)$ soit une dérivée approximativement semi-continue inférieurement, $\phi_{2}(x)$ une dérivée approximativement semi-continue supérieurement.

IX. Peut-on pour $E \neq 0, E \in F_{\sigma}$, formuler la condition $M_{4}$ de la manière suivante: pour tout $x \in E$ il existe $\eta_{x}>0$ tel qu'il résulte de $I \rightarrow x,|I|^{-1} \cdot|E I|$ $\leqq \eta_{x} q u e \lim |I| \cdot \operatorname{dist}(x, I)^{-1}=0$.

Ce serait possible s'il existait une telle suite $\left\{\phi_{n}\right\}$ des ensembles $F_{\sigma}$ et des nombres $a_{n}>0$ que $E=\sum_{n=1}^{\infty} \phi_{n}$ et $\eta_{x} \geqq a_{n}$ pour tout $x \in \phi_{n}$. En prenant la fonction de mesure $\phi(x)$ de l'ensemble $E$ (continue comme on le sait), on peut déterminer $\eta_{x}$ au moyen de passages répétés aux limites inférieures de certains quotients des différences de la fonction $\phi(x)$, de même que la dérivée inférieure. Je n'ai réussi, cependant, qu'a montrer que les ensembles $\left\{\eta_{x} \geqq a\right\}$ sont du type $F_{\sigma \delta}$. 


\section{BIBLIOGRAPHIE}

1. V. Bogomolova, Sur une classe des fonctions asymptotiquement continues (Russian, French summary), Rec. Math. (Mat. Sbornik) vol. 32 (1924) pp. 152-171.

2. T. Brodén, Derivierbare Funktionen mit ïberall dichten Maxima und Minima, Öfv. Vet. Ak. Förhandl. Stockholm vol. 57 (1900) pp. 423-441.

3. - Fortgesetzte Untersuchungen über derivierbare Funktionen mit überall dichten Maxima und Minima, Öfv. Vet. Ak. Förhandl. Stockholm vol. 57 (1900) pp. 743-761.

4. A. Brudno, Continuity and differentiability (Russian, English summary), Rec. Math. (Mat. Sbornik) N.S. vol. 13 (1943) pp. 119-134.

5. G. Choquet, Application des propriétés descriptives de la fonction contingent à la théorie des fonctions de variable réelle et à la géométrie différentielle des variêtés cartésiennes (Thèse), J. Math. Pures Appl. (9) vol. 26 (1947) pp. 115-226 (1948).

6. J. A. Clarkson, $A$ property of derivatives, Bull. Amer. Math. Soc. vol. 53 (1947) pp. 124125.

7. A. Denjoy, Sur les fonctions dérivées sommables, Bull. Soc. Math. France vol. 43 (1915) pp. $161-248$.

8. - Sur une propriété des fonctions dérivées, L'Enseignement Mathéma tique vol. 18 (1916) pp. 320-328.

9. G. Goldovsky, Note sur les dérivées exactes, Rec. Math. (Mat. Sbornik) vol. 35 (1928) pp. $35-36$.

10. H. Hahn, Über den Fundamentalsatz der Integralrechnung, Monatshefte für Mathematik vol. 16 (1905) pp. 161-166.

11. S. Kempisty, Sur les fonctions dérivées bornées, Annales de la Société Polonaise de Mathématique vol. 3 (1925) pp. 88-91.

12. A. Köpcke, Ueber Differentiirbarkeit und Anschaulichkeit der stetigen Functionen, Math. Ann. vol. 29 (1887) pp. 123-140.

13. - Ueber eine durchaus differentiirbare stetige Function mit Oscillationen in jedem Intervalle, Math. Ann. vol. 34 (1889) pp. 161-171.

14. - Analytische Darstellung einer differentiirbaren Function mit Oscillationen in jedem Intervalle, Mitteilungen der Mathematischen Gesellschaft in Hamburg vol. 2 (1890) pp. 128-153.

15. - - Ein Satz über Functionen mit Oscillationen in jedem Intervalle, Mitteilungen der Mathematischen Gesellschaft in Hamburg vol. 3 (1899) pp. 376-379.

16. A. Kronrod, Sur la structure de l'ensemble des points de discontinuité d'une fonction dérivable en ses points de continuité (Russian, French summary), Bull. Acad. Sci. URSS Sér. Math. (1939) pp. 569-578.

17. I. Maximoff, On approximately continuous functions, Bull. Amer. Math. Soc. vol. 45 (1939) pp. 264-268.

18. - On density points and approximately continuous functions, Tôhoku Math. J. vol. 47 (1940) pp. 237-250.

19. S. Mazurkiewicz, Konstrukcya funkcji różniczkowalnej, mającej wszędzie gęsty zbiór przedziałow statosci, Prace Matematyczno-Fizyczne vol. 27 (1916) pp. 87-91.

20. - O budowie funkcyj rózniczkowalnych, pantachicznie oscylujacych I (Polish, French summary), Comptes Rendus des Séances de la Société des Sciences et des Lettres de Varsovie, classe III vol. 10 (1917) pp. 1167-1184.

21. - Budowa funkcyj różniczkowalnych, pantachicznie oscylujqcych II (Polish, French summary); Sur les fonctions dérivables admettant un ensemble partout dense de maxima et minima II, Comptes Rendus des Séances de la Société des Sciences et des Lettres de Varsovie. Classe III vol. 11 (1918) pp. 267-279. 
22. J. Pereno, Sulle funzioni derivabili in ogni punto ed infinitamente oscillanti in ogni intervallo, Giornale di Matematiche di Battaglini vol. 35 (1897) pp. 137-149.

23. D. Pompéiu, Sur les fonctions dérivées, Math. Ann. vol. 63 (1906) pp. 326-332.

24. S. Ruziewicz, O funkcyach ciqglych, monotonicznych, posiadajacych pantachiczne przedziaty stalosci, Prace Matematyczno-Fizyczne vol. 27 (1916) pp. 19-31.

25. - Sur les fonctions qui ont la même dérivée et dont la différence n'est pas constante, Fund. Math. vol. 1 (1920) pp. 148-151.

26. - O O niestosowalnosci zasadniczego twierdzenia rachunku catkowego do funkcyj majacych pochodne nieskończone (Sur l'inapplicabilité du théorème fondamental du calcul intégral aux fonctions à dérivées infinies), Prace Matematyczno-Fizyczne vol. 31 (1920) pp. 31-34. 1937.

27. S. Saks, Theory of the integral, Monografje Matematyczne, vol. 7, Warszawa-Lwow,

28. A. Schoenflies, Über unendlich oft oszillierende Funktionen, Königsb. Phys. Ökon. Ges. vol. 41 (1900) pp. 11-13.

29. ——Über die ïberall oszillierenden differenzierbaren Funktionen, Math. Ann. vol. 54 (1901) pp. 553-563.

30. N. A. Selivanov, Démonstration d'un théorème de $M$. A. Denjoy sur les fonctions dérivées (Russian, French summary), Mitteilungen des Forschungsinstituts für Mathematik und Mechanik an der Kujbyschew-Universität Tomsk vol. 2 (1938) pp. 29-31.

31. G. Tolstoff, Sur quelques propriétés des fonctions approximativement continues, Rec. Math. (Mat. Sbornik) N.S. vol. 5 (1939) pp. 637-645.

32. L. Tonelli, Sulle derivate esatte, Memorie della Accademia delle Scienze dell'Istituto Bologna (8) vol. 8 (1930-1931) pp. 13-15.

33. Z. Zahorski, Konstrukcja funkcji różniczkowalnej, monotonicznej, nie statej, majqcej wszędziegęsty zbiór przedziatów statošci. Über die Konstruktion einer differenzierbaren, monotonen, nicht konstanten Funktion, mit ïberall dichter Menge von Konstanzintervallen (German, Polish summary), Comptes Rendus des Séances de la Société des Sciences et des Lettres de Varsovie, classe III vol. 30 (1937) pp. 202-206.

34. — O mnożestwie tocek niediferencirujemosti nieprerywnoj funkcii, Rec. Math. (Mat. Sbornik) N.S. vol. 9 (1941) pp. 487-510. 401 .

35. ——, Sur les intégrales singulières, C. R. Acad. Sci. Paris vol. 223 (1946) pp. 399-

36. - Sur les dérivées des fonctions partout dérivables, C. R. Acad. Sci. Paris vol. 223 (1946) pp. 415-417.

37. - Sur les ensembles des points de divergence de certaines intégrales singulières, Annales de la Société Polonaise de Mathématique vol. 19 (1947) pp. 66-105.

38. — Über die Menge der Punkte in welchen die Ableitung unendlich ist, Tôhoku Math. J. vol. 48 (1941) pp. 321-330.

39. Z. Zalcwasser, O funkcjach Köpckego (Polish, French summary), Prace MatematycznoFizyczne vol. 35 (1929) pp. 57-99.

UNIVERSITY OF ŁóDź, Łódź, Poland. 